Portland State University

PDXScholar

Spring 5-22-2015

\title{
Workplace Aggression: A Multi-Study Examination of Work and Nonwork Consequences
}

\author{
Caitlin Ann Demsky \\ Portland State University
}

Follow this and additional works at: https://pdxscholar.library.pdx.edu/open_access_etds

Part of the Applied Behavior Analysis Commons, and the Industrial and Organizational Psychology Commons

Let us know how access to this document benefits you.

\section{Recommended Citation}

Demsky, Caitlin Ann, "Workplace Aggression: A Multi-Study Examination of Work and Nonwork Consequences" (2015). Dissertations and Theses. Paper 2338.

https://doi.org/10.15760/etd.2335

This Dissertation is brought to you for free and open access. It has been accepted for inclusion in Dissertations and Theses by an authorized administrator of PDXScholar. Please contact us if we can make this document more accessible: pdxscholar@pdx.edu. 
Workplace Aggression: A Multi-Study Examination of Work and Nonwork Consequences

by

\title{
Caitlin Ann Demsky
}

\begin{abstract}
A dissertation submitted in partial fulfillment of the requirements for the degree of
\end{abstract}

\author{
Doctor of Philosophy \\ in \\ Applied Psychology
}

\author{
Dissertation Committee: \\ Charlotte Fritz, Chair \\ Donald M. Truxillo \\ Lauren S. Simon \\ Wayne W. Wakeland
}

Portland State University

2015 
(C) 2015 Caitlin Ann Demsky 


\begin{abstract}
Workplace aggression has been associated with a number of detrimental employee and organizational outcomes, both at work and away from work. This dissertation includes three studies that expand our knowledge of the implications of workplace aggression in the work and nonwork domains. Further, this research illuminates the processes through which this relationship occurs by utilizing various sources of data from employees in a variety of contexts including universities, long term health care, and the USDA Forest Service. In Study 1, which was published in the Journal of Occupational Health Psychology, multi-source data are utilized to identify the indirect effects of coworkerreported workplace aggression on self and significant-other reported work-family conflict via self-reported psychological detachment from work. Study 2 identifies an indirect effect of workplace aggression on parental warmth via increased perceived stress utilizing longitudinal data from the Work, Family, and Health Network. Finally, Study 3 utilizes data from the USDA Forest Service to examine associations between workplace aggression and safety outcomes. Workplace aggression was found to be associated with increased resource depletion (i.e., rumination, cognitive failure) and decreased workplace safety (i.e., increased workplace accidents, decreased safety compliance). Workplace aggression was indirectly associated with safety participation and workplace injuries via cognitive failure and rumination, respectively. Safety climate, an organizational resource, moderated the relationship between rumination and safety behaviors. Finally, the indirect effect of coworker aggression on safety compliance via rumination was found to be conditional on low levels of safety climate, while the indirect effect of supervisor
\end{abstract}


aggression on safety participation via rumination was also found to be conditional on low levels of safety climate. The current body of work provides implications for developing workplace interventions to reduce negative outcomes of workplace aggression, such as general stress management and recovery from work interventions. Several avenues for future research are suggested as well, including examining objective health outcomes of workplace aggression, utilizing longitudinal designs, and identifying additional moderators of the association between workplace aggression and employee outcomes. 


\section{Acknowledgements}

There are a number of individuals to whom I owe countless thanks for their support throughout this process. I would like to express my immense gratitude to my dissertation chair and advisor, Dr. Charlotte Fritz, for her mentorship and unwavering support throughout my graduate career. I would also like to thank my dissertation committee members, Drs. Donald Truxillo, Lauren Simon, and Wayne Wakeland, for their support, feedback, and guidance throughout this process. Finally, I would like to thank Dr. Leslie Hammer for her support and guidance over the past five years.

I am also incredibly grateful for my partner, friends, family, and colleagues, who have provided an endless amount of support, encouragement, guidance, and much-needed distraction throughout the years. Though this process was not often easy, each of you contributed to making it quite an enjoyable and memorable endeavor.

Finally, I would like to acknowledge the National Science Foundation for providing me with a generous source of funding through a Graduate Research Fellowship, which has allowed me a great deal of academic and intellectual freedom over the past three years. I would like to acknowledge the Work, Family, and Health Network for providing access to the data found in Study 2, and would also like to thank my collaborators at the USDA Forest Service for their time, energy, and participation in Study 3 of this dissertation. 
Table of Contents

Acknowledgements iii

List of Tables .. V

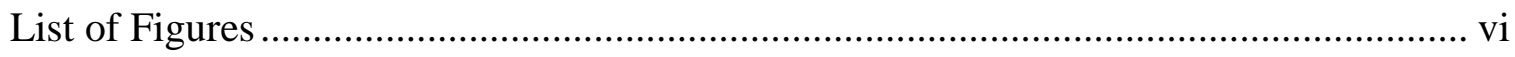

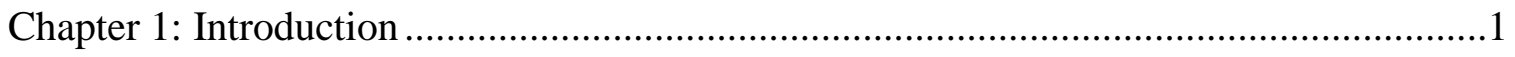

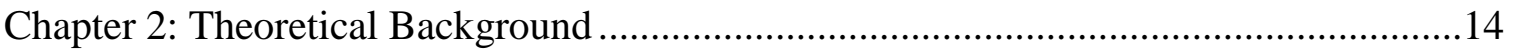

Chapter 3: Shrugging it Off: Does Psychological Detachment from Work Mediate the

Relationship between Workplace Aggression and Work-Family Conflict?.....................21

Chapter 4: Extending the Examination of Nonwork Outcomes of Workplace Aggression:

A Longitudinal Examination

Chapter 5: The Role of Perceived Stress in the Relationship between Workplace Aggression and Parenting: Evidence from the Work, Family, \& Health Network

Chapter 6: Examining Understudied Work Outcomes of Workplace Aggression: Associations with Safety.

Chapter 7: Workplace Aggression and Occupational Safety: A Moderated Mediation

Model.....

Chapter 8: General Discussion.

References

Appendix: Items for Study \#3 


\section{List of Tables}

Table 3.1 Means, Standard Deviations, and Correlations among Study Variables

Table 3.2 Hierarchical Regression Analyses Predicting WFC from Workplace Aggression

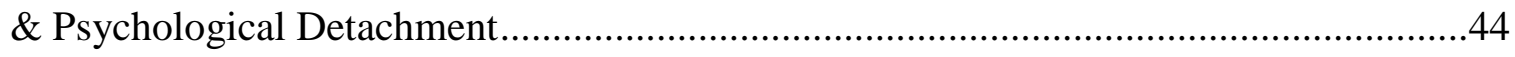

Table 3.3 Bootstrapped Mediation Analyses ...........................................................46

Table 5.1 Means, Standard Deviations, and Correlations among Study Variables ...........74

Table 5.2 Bootstrapped Mediation Analyses ........................................................... 76

Table 7.1 Means, Standard Deviations, and Correlations among Study Variables .........116

Table 7.2 Hypothesis 4: Bootstrapped Mediation Analyses .......................................122

Table 7.3 Summary of Results.............................................. 129 


\section{List of Figures}

Figure 1.1 Summary model of the relationships examined in the enclosed dissertation...10

Figure 3.1 Hypothesized relationships among study variables

Figure 4.1 Summary model of the proposed relationships examined in Study 2. .56

Figure 5.1 Hypothesized relationships among study variables.

Figure 6.1 Summary model of the proposed relationships examined in Study 3. .84

Figure 7.1 Diagram of proposed moderated mediation model.

Figure 7.2 Exploratory analysis: Relationship between coworker aggression and safety compliance, moderated by cognitive failure.

Figure 7.3 Hypothesis 6e. The relationship between rumination and workplace accidents moderated by safety climate.

Figure 7.4 Hypothesis $6 \mathrm{~g}$. The relationship between rumination and safety compliance moderated by safety climate. 125 Figure 7.5 Hypothesis 6h: The relationship between rumination and safety participation moderated by safety climate. 125 


\section{Chapter 1: Introduction}

Recent estimates suggest that as many as $40 \%$ of U.S. employees have experienced some form of psychological aggression at work in the last 12 months (Schat, Frone, \& Kelloway, 2006). Additional estimates place the prevalence of experienced workplace aggression as high as $96 \%$ of workers (Porath \& Pearson, 2010). Workplace aggression has been identified as a serious and prevalent phenomenon with important implications for its victims. Researchers have defined workplace aggression as "efforts by individuals to harm others with whom they work, or have worked, or the organizations in which they are currently, or were previously, employed" (Neuman \& Baron, 1997, p. 38). In addition to being a common workplace stressor, workplace aggression has been associated with a number of deleterious outcomes for both employees and organizations alike, including decreased job satisfaction, affective commitment, physical well-being, and job performance, as well as increased turnover intentions, emotional exhaustion, depression, interpersonal and organizational deviance (Herschovis \& Barling, 2010).

\section{Outcomes of Experienced Workplace Aggression}

As will be detailed in these three studies, workplace aggression has been associated with a wide number of negative employee and organizational outcomes. It is important to note that the literature being reviewed herein refers to experienced workplace aggression, or the effects of workplace aggression on those employees who fall victim to this workplace stressor, as opposed to focusing on those who enact workplace aggression. While much of the earlier literature on workplace aggression 
focused on work-related outcomes, recent research has begun to call attention to nonwork outcomes associated with workplace aggression as well. A brief review of the outcomes of workplace aggression is first necessary to draw attention to the importance of examining this construct further and identifying ways to prevent and mitigate workplace aggression. The following paragraphs provide a review of the effects of workplace aggression on three types of outcomes: work, well-being, and nonwork.

As mentioned above, meta-analytic evidence has linked workplace aggression to attitudinal, behavioral, and well-being outcomes. Specifically, experiencing workplace aggression has been associated with decreased job satisfaction, coworker satisfaction, supervisor satisfaction, affective commitment, perceptions of organizational justice, job performance, and organizational citizenship behaviors, as well as increased turnover intentions, absenteeism, and both interpersonal and organizational deviance (Bowling \& Beehr, 2006; Fox, Spector, \& Miles, 2001; Herschovis \& Barling, 2010; Lim, Cortina, \& Magley, 2008). These findings suggest that workplace aggression has serious consequences for both employees and organizations alike.

In addition to work-related outcomes, workplace aggression has also been associated with a number of health and well-being outcomes in its victims. Workplace aggression is a particularly troublesome workplace stressor, as it is often perceived as being out of the victim's control (Schat \& Kelloway, 2000). Meta-analytic evidence has shown that workplace aggression is associated with decreased psychological well-being, and increased anxiety, depression, frustration, job stress, and doctor's visits (Hershcovis \& Barling, 2010; Spector \& Jex, 1998). Longitudinal evidence has linked problematic workplace interpersonal relationships to increased depression levels over two years 
(Stoetzer et al., 2009). Additionally, Frone (1999) has linked workplace aggression to increased alcohol use.

In addition to having a detrimental effect on employees and organizations, recent research has demonstrated the potential for workplace aggression to have wide-reaching negative effects on employees' nonwork lives. Various forms of workplace aggression have been associated with decreased functioning in the nonwork domain, evident in outcomes such as increased marital withdrawal and anger (Brodsky, 1976; Repetti, 1989; Saxbe, Repetti, \& Graesch, 2011; Story \& Repetti, 2006). Additionally, Restubog, Scott, and Zagenczyk (2011) recently found that abusive supervision was associated with higher levels of spousal undermining. This relationship was found to be mediated via increased psychological distress. Other researchers have found that surface acting and burnout mediate the relationship between workplace aggression and work-family conflict (Carlson, Ferguson, Hunter, \& Whitten, 2012). Still other researchers have drawn upon the literature on recovery from work demands to identify a lack of psychological detachment as a mediator of the relationship between workplace aggression and workfamily conflict (Study \#1: Demsky, Ellis, \& Fritz, 2014).

\section{Alternative Methods of Conceptualizing and Measuring Workplace Aggression}

A number of terms exist for identifying various related constructs that fall under the general umbrella of workplace aggression. For example, such constructs include workplace bullying (Rayner, 1997), abusive supervision (Tepper, 2000), incivility (Andersson \& Perason, 1999), and interpersonal conflict (Spector \& Jex, 1998). The specific definitions of each of these constructs tends to vary in terms of intent, intensity, frequency, perceived invisibility, and power dynamics (cf. Hershcovis, 2011). Hershcovis 
recently argued that it is unclear whether or not the proliferation of such constructs has added value to the literature, and proposed that researchers label such related constructs under the umbrella of 'workplace aggression,' while examining such defining characteristics as potential moderators.

Clarifying constructs. In her call to clarify how individual constructs of workplace aggression were adding substantially to the literature, Hershcovis (2011) provided evidence that several of the most commonly studied aggression constructs had similar patterns of relationships with a number of outcome variables. Her examination included the constructs of abusive supervision, bullying, social undermining, incivility, and interpersonal conflict. Results of a supplementary meta-analysis revealed similar relationships amongst these constructs and the outcomes of job satisfaction, turnover intentions, psychological well-being, physical well-being, and affective commitment.

Whereas there are a number of related constructs, it is worth noting the points at which definitions of some of these constructs diverge from one another. Abusive supervision has been defined as the "sustained display of hostile verbal and non-verbal behaviors, excluding physical contact" (Tepper, 2000, p. 178). Workplace bullying has been described as exposure to repeated negative acts from supervisors, coworkers, or subordinates (e.g., teasing, social exclusion, or ridicule; Einarsen, 2000). Social undermining refers to repeated behaviors over time, which are intended to interfere with the ability to establish and maintain positive relationships, work-related success, and a positive reputation (Duffy, Ganster, \& Pagon, 2002). Workplace incivility refers to low intensity deviant acts (e.g., rude verbal and nonverbal behaviors) with ambiguous intent to harm (Andersson \& Pearson, 1999). Finally, workplace interpersonal conflict has been 
described as an organizational stressor which can be either overt or covert, involving disagreements between employees (Spector \& Jex, 1998). Hershcovis (2011) identified several distinguishing characteristics of these definitions and called for their examination as moderators in future studies of workplace aggression. These potential moderators include: intent, intensity, frequency, perceived invisibility, perpetrator-victim relationship (e.g., power).

Though often generally defined, workplace aggression may come from a number of different sources within the work context. As Barling, Dupre, and Kelloway (2009) describe, there are four forms of aggression. Type I aggression refers to those acts in which the perpetrator has no legitimate relationship with the target. Type II aggression occurs when the perpetrator has a legitimate relationship with the organization. Aggression from customers and clients fall into this category. Type III aggression refers to those acts in which the perpetrator is an organizational insider, which would include aggressive acts from either current or former employees. Finally, Type IV aggression refers to acts in which the perpetrator has an ongoing or previous relationship with an employee of the organization. This includes aggressive acts from family, friends, or spouses.

In the present studies, the term workplace aggression is used to refer broadly to negative acts in the workplace that harm other employees or the organization, and includes terms such as interpersonal conflict, psychological aggression, and workplace incivility. When reviewing relevant literature, the specific construct name used by the original researchers is used to enhance clarity where necessary. 
Measuring aggression. Similar to concerns of multiple labels that refer to the construct of workplace aggression, there are also a number of ways of measuring workplace aggression. Specifically, researchers have noted the necessity of measuring the source from which workplace aggression occurs, though for a number of reasons, this is often not done in practice. While several constructs specify the source of workplace aggression, such as abusive supervision (e.g., Tepper, 2000), a number of workplace aggression constructs refer to experienced aggression from anyone in the organization. Researchers have stressed the importance of identifying the source of aggression when surveying individuals, as recent evidence suggests that some outcomes of workplace aggression may vary by source, suggesting a different nomological network for each, and hence their conceptual distinctiveness. For example, Hershcovis and Barling (2010) meta-analytically reviewed attitudinal, behavioral, and health-related outcomes of aggression based on perpetrator source (i.e., supervisors, coworkers, and outsiders). The results of their analyses suggest that supervisor aggression has the most serious detrimental effects across both attitudinal (i.e., job satisfaction, affective commitment, turnover intentions) and behavioral outcomes (i.e., interpersonal deviance, organizational deviance), while coworker aggression exerted stronger effects than outsider aggression on attitudinal and behavioral outcomes. In general, no significant differences were found between sources of aggression for health-related outcomes (e.g., psychological distress, emotional exhaustion, depression, and physical well-being).

Workplace aggression researchers have been called to examine sources of aggression (Hershcovis \& Barling, 2010). Although Studies 1 and 2 of this dissertation do not include information on the source of aggression, Study 3 seeks to remedy this 
limitation by gathering information on the source of workplace aggression (i.e., supervisors and coworkers). The hypothesized effects in Study 3 are not theorized to be different based on source of aggression, nevertheless, the effects of both supervisor and coworker aggression will be examined.

\section{Relevance to the field of Occupational Health Psychology}

Occupational health psychology (OHP) refers to "the application of psychology to improving the quality of work life, and to protecting and promoting the safety, health, and well-being of workers" (NIOSH, 2010). The study of workplace aggression has been viewed as falling under the realm of OHP, as the experience of workplace aggression is directly associated with employee health and well-being (Herschovis \& Barling, 2010). Reducing the prevalence of workplace aggression speaks directly to improving the quality of work life for employees as well. Recently, the Centers for Disease Control and Prevention has identified a focus on Total Worker Health ${ }^{\mathrm{TM}}$ (TWH; CDC, 2015), which refers to a strategy aimed at the integration of occupational safety and health protection and health promotion practices in an effort to both prevent employee injuries and illnesses, as well as to advance employee health and well-being. In a recent review, Hammer and Sauter (2013) discussed the importance of implementing workplace interventions at both the organizational and individual levels in an effort to reduce negative effects of work-life stress. The enclosed three studies are directly relevant to this aim, as each provides implications for interventions at the organizational and individual levels, with the goal of reducing the negative effects of workplace aggression on work and nonwork outcomes. A recent overview of TWH interventions found that TWH interventions were more effective in reducing risk factors for injuries and chronic 
illnesses than workplace interventions with more narrow foci (Anger et al., 2014). Through the study of the antecedents, outcomes, mechanisms, and moderators of workplace aggression, researchers can identify strategies for both reducing the occurrence of workplace aggression, as well as strategies for reducing the negative effects of workplace aggression.

\section{Statement of Purpose}

The proposed dissertation makes several contributions to the literature surrounding workplace aggression. First, while a few studies have noted a relationship between workplace aggression and increased work-family conflict, Study 1 was the first to identify psychological detachment from work as a specific mechanism through which workplace aggression may be negatively associated with the nonwork domain (Demsky et al., 2014). Secondly, little is currently known about how workplace aggression may be associated with detrimental outcomes for family members other than the target employee or spouse (e.g., Restubog et al., 2011), a gap this dissertation seeks to fill. Specifically, Study 2 employs a longitudinal approach to examine how workplace aggression may influence employee parents' interactions with their children. Third, while workplace aggression has been associated with a number of detrimental work outcomes, few studies have addressed the relationship between workplace aggression and safety outcomes, a gap in the literature that will be addressed by the third and final study. Finally, Study 3 addresses possible moderators of the relationship between workplace aggression and safety outcomes. The moderators, mindfulness and safety climate, are individual and organization-level resources that are capable of being influenced and trained (KabatZinn, 1994; Zohar, 2003). 
Through three independent studies, the proposed dissertation seeks to examine several work and nonwork consequences of workplace aggression, mechanisms through which these relationships occur, and both personal and work-related resources that may buffer the negative influence of workplace aggression on employees. By gaining an increased understanding of the process through which workplace aggression is associated with potential outcomes, in addition to key factors that may lessen the negative impact of workplace aggression, interventions may be designed and implemented to address this workplace stressor. The ensuing studies are motivated by the following related research questions:

1) Does psychological detachment from work serve as a potential mechanism through which workplace aggression is associated with increased work-family conflict?

2) Does workplace aggression spill over to the nonwork domain to impact parent-child interactions?

3) Does workplace aggression influence safety in the workplace? By what mechanisms does this occur? What moderators may inhibit this process?

\section{Summary and Proposed Studies}

Although a great deal of research has focused on examining antecedents and outcomes of workplace aggression over the last decade, there are still several key gaps remaining in the literature, which the current dissertation seeks to fill. Namely, we still do not have a clear understanding of how workplace aggression may spill over to the nonwork domain to impact employee outcomes outside of work. Studies 1 and 2 explore 
this process in more detail. Study 3, discussed below, addresses the relationship between workplace aggression and safety outcomes, as well as potential individual and organizational-level moderators of this relationship. A summary model of the proposed relationships tested in the following dissertation can be found in Figure 1.1.

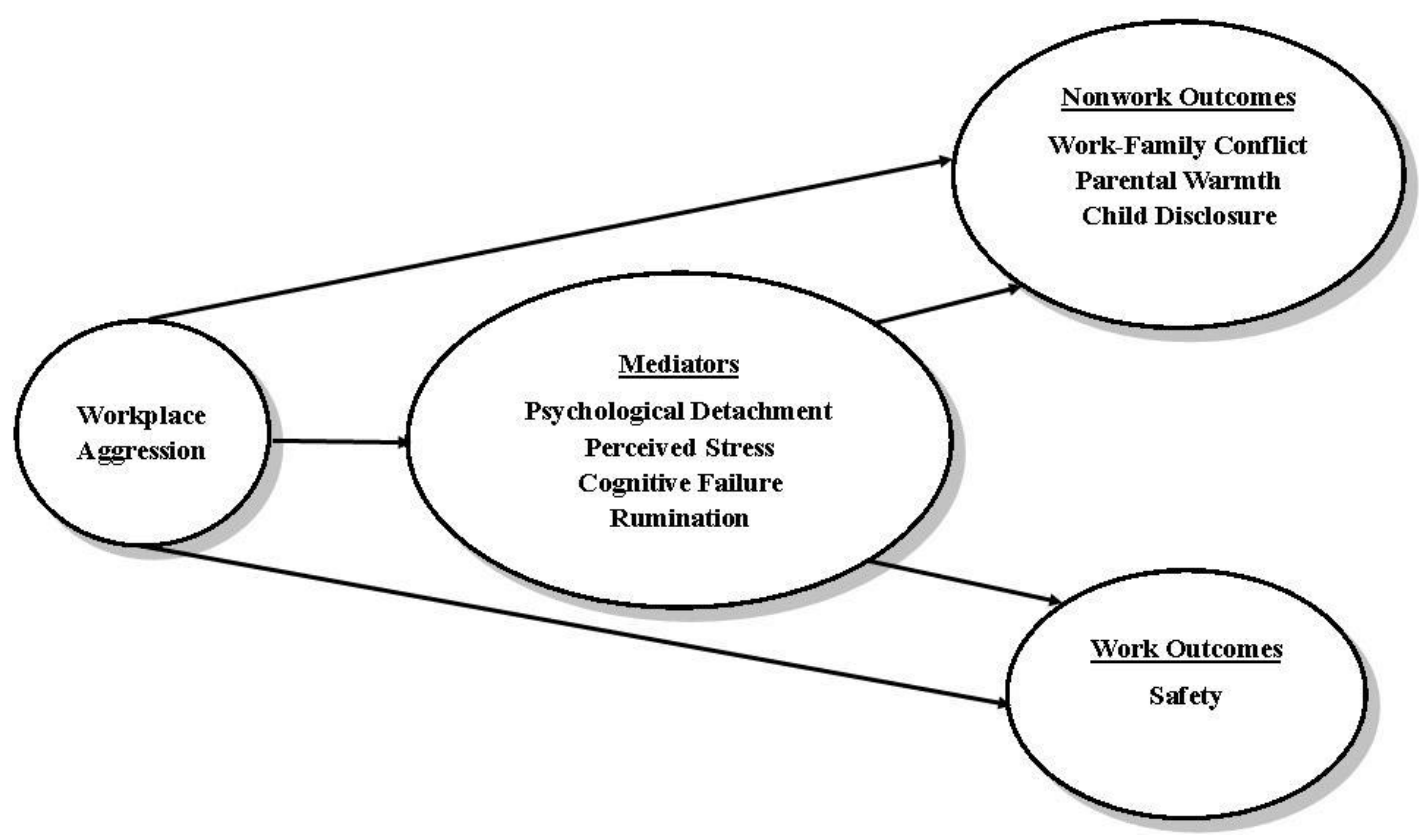

Figure 1.1 Summary model of the proposed relationships examined in the enclosed dissertation.

In Study 1, which was published in the Journal of Occupational Health Psychology (Demsky et al., 2014), my colleagues and I examine the process through which workplace aggression is associated with increased work-to-family conflict through decreased psychological detachment from work during nonwork time. Utilizing a multisource, cross-sectional sample of university employees, the relationship between coworker-reported workplace aggression and both self-reported and significant-otherreported work-family conflict was found to be mediated by self-reported psychological detachment. Participants in this sample were employed in a variety of academic roles, 
including administrative assistants, web developers, program coordinators, directors, and library associates. University employees interact with a variety of individuals, including supervisors, coworkers, and members of the public, and as such, are likely to experience workplace aggression (Björkqvist, Österman, \& Hjelt-Bäck, 1994). Thus, this study addresses the first research question by examining one potential mechanism through which workplace aggression is associated with nonwork outcomes.

Study 2 (Demsky\& Hammer, in progress) extends this spillover process to examine the association between workplace aggression and parent-child interactions. Much of the research surrounding workplace aggression has, rightfully so, focused on the detrimental effects of workplace aggression on both the target employee (e.g., Hershcovis \& Barling, 2010) and a significant other (Green, Schaefer, MacDermid, \& Weiss, 2011; Repetti, 1989; Story \& Repetti, 2006). However, children may also be negatively impacted by the workplace demands parents face each day. This study utilized longitudinal data from the Work, Family, and Health Network (Bray et al., 2013) to address the second proposed research question. That is, does workplace aggression spill over to the nonwork domain to influence parent-child interactions? Hypotheses are tested using a sample of long-term healthcare employees (e.g., licensed practical nurses, registered nurses, and licensed or certified nurse assistants). Healthcare employees are exposed to high levels of workplace aggression - both psychological and physical - and as such, have been the focus of much workplace aggression research (Bureau of Labor Statistics, 2010; Gerberich et al., 2004). Results indicate significant indirect effects of workplace aggression on parental warmth twelve months post-baseline via increased perceived stress. 
As a final contribution, Study 3 of this dissertation addresses the third proposed research question by examining the relationship between workplace aggression and safety outcomes, as well as possible mediators and moderators of this relationship (Demsky \& Fritz, in progress). Specific related hypotheses were tested utilizing a large-scale crosssectional sample of United States Department of Agriculture (USDA) Forest Service employees through a funded two-year project. While workplace aggression has been associated with a number of detrimental employee and organizational outcomes (e.g., Hershcovis \& Barling, 2010), much less is known about the potential effects of workplace aggression on workplace safety. Study 3 proposes that experiences of workplace aggression may be associated with increased accidents and injuries, and decreased safety behavior (i.e., safety participation and compliance) through increased cognitive failure and rumination. Additionally, it is proposed that the presence of individual and workplace resources may mitigate the negative relationship between workplace aggression, cognitive failure and rumination, and workplace safety. Specifically, mindfulness is proposed as an individual resource that may weaken the negative relationship between workplace aggression and resource loss, while safety climate is proposed as an organizational resource that may weaken the relationship between resource loss and safety outcomes.

USDA Forest Service employees are a particularly well-suited group of participants with which to examine the hypotheses proposed in Study 3. Many jobs within the Forest Service are inherently dangerous, particularly in the area of wildland firefighting. Since 2000, 137 federal or volunteer wildland firefighters have died in the line-of-duty, with 35 of those fatalities occurring in the states our present sample are 
employed in. Fatal incidents have included crashes (i.e., aviation or vehicle), heart attacks, entrapment, and being struck by a tree. Limited data on non-fatal injuries suggest that the most common injuries associated with wildland firefighting include sprains, slips/trips/falls, and contact with equipment (Butler, Scott, \& Sussell, 2014).

Additionally, the Forest Service has recently drawn negative attention in the media for claims of harassment and abuse by female employees (Nixon, 2014). Finally, the USDA Forest Service has invested in employee well-being trainings and safety initiatives (Rocky Mountain Research Station Human Factors \& Risk Management, n.d.) that indicate an environment ripe for examining some of the personal and organizational resources that may attenuate the negative relationship between workplace aggression and safety. 
Chapter 2: Theoretical Background

A number of theories have been used to explain the occurrence of workplace aggression, as well as the relationships between workplace aggression and work and health outcomes. The current dissertation draws primarily from three theoretical frameworks: Conservation of Resources theory (Hobfoll, 1989), the Work-Home Resources Model (ten Brummelhuis \& Bakker, 2012), and Job Demands-Resources theory (Demerouti, Bakker, Nachreiner, \& Schaufeli, 2001). Below, I discuss these theories as they relate to the research questions described in Chapter 1.

Conservation of Resources (COR) theory. COR theory suggests that individuals are motivated to maintain, build, and protect personal resources (Hobfoll, 1989). These resources are said to be objects, conditions, personal characteristics, or energies that an individual values or that serve as a means for obtaining additional resources. According to COR theory, individuals experience stress when resources are threatened, lost, or fail to be regained after an investment of resources. Additionally, the two driving principles of COR theory are that 1) resource loss is more salient than resource gain, and 2) resources must be invested to gain further resources, to protect from losing resources, or to recover from resource loss (Hobfoll, 1998, 2001). In this regard, the experience of workplace aggression can be seen as a form of resource loss, or a threat to one's existing resources.

The ongoing experience of such aggression may contribute to the actual loss of resources, and to a chronic degree, a resource loss spiral (Demerouti, Bakker, \& Bulters, 2004; Hobfoll, 2001). Loss spirals are theorized to occur when individuals do not have sufficient resources to stop further resource loss, or to protect remaining resources 
(Hobfoll, 1989). This loss of resources due to workplace aggression becomes evident in reduced health and well-being, as well as decreased functioning in the work and nonwork domains. Those individuals with a deficit of resources may be more likely to employ unsuccessful coping strategies in an attempt to halt resource loss. However, interrupting these loss spirals is critical in halting further resource loss.

In a recent review of COR theory, a number of specific psychological resources were reviewed as having been reported in the organizational literature (Halbesleben, Neveu, Paustian-Underdahl, \& Westman, 2014). Two relevant and commonly studied psychological resources are social support and recovery experiences. In the context of the current proposed dissertation, workplace aggression may be seen as a threat to one's resources, such as social support, information, or self-esteem. One such mechanism that may halt the process of resource loss is psychological detachment from work. Psychological detachment from work has been defined as one's "sense of being away from the work environment" (Etzion, Eden, \& Lapidot, 1998, p. 579). Engaging in psychological detachment refers to both a physical and mental separation from one's work. Psychological detachment from work is one of four recovery experiences previously identified by Sonnentag \& Fritz (2007). Recovery from work demands refers to a process in which an individual's systems that were called upon while addressing work demands are allowed to return to prestressor levels (Meijman \& Mulder, 1998). While experiencing workplace aggression may deplete one's resources (e.g., emotional, cognitive), engaging in recovery from work - specifically, psychological detachment may halt this process of resource loss. However, it is likely that chronic exposure to workplace aggression may inhibit one's ability to psychologically detach from work, as 
workplace aggression has previously been associated with rumination and increased activation (Keenan \& Newton, 1985). Failure to halt the process of resource loss may be associated with the spillover of negative workplace experiences (i.e., workplace aggression) to the nonwork domain.

In addition to psychological detachment, Study 3 in the current dissertation proposes other resources that may prevent resource loss as a result of workplace aggression: mindfulness and safety climate. Mindfulness, defined as "paying attention in a particular way: on purpose, in the present moment, nonjudgmentally" (Kabat-Zinn, 1994, p. 4), is proposed as an individual-level resource that may mitigate the negative association between workplace aggression and resource loss, conceptualized as cognitive failure and rumination. Recent research has identified mindfulness as a psychological resource that is negatively associated with vicarious trauma and psychosomatic symptoms in firefighters (Setti \& Argentero, 2014). Additionally, meta-analytic evidence suggests that mindfulness interventions have positive effects on both mental and physical health (Grossman, Niemann, Schmidt, \& Walach, 2004).

Safety climate refers to employee perceptions of safety-related practices, procedures, and policies, and the types of safety-related behaviors that are rewarded, supported, and expected by management (Zohar, 1980). High levels of safety climate have been linked to fewer accidents and injuries, and to increased safety behaviors (i.e., safety compliance and safety participation; Nahrgang, Morgeson, \& Hofmann, 2011). In Study 3, I propose that experiencing workplace aggression may be associated with diminished resources that are needed to attend to important workplace safety practices. In the event of diminished personal resources, I propose that a strong safety climate may 
serve as an organizational resource that serves as a reminder of the importance of engaging in critical workplace safety behaviors.

Work-Home Resources Model. Building on COR theory, ten Brummelhuis and Bakker (2012) recently proposed the Work-Home Resources Model. This model was proposed to address several limitations of the existing work-family literature, including “(a) What are the causal processes behind work-family conflict and enrichment? (b) Is work-family enrichment or conflict more likely under certain macro conditions and among employees with certain personality traits? and (c) How do work-home processes develop over time?” (p. 546). In their description of the Work-Home Resources Model, ten Brummelhuis and Bakker draw upon categories of resources described by Hobfoll (2002) to explain the process by which work may enrich or conflict with the nonwork domain.

As the concepts of resources and resource loss are directly relevant to the enclosed manuscripts, it is worthwhile to review the definitions of resources as outlined by these researchers. ten Brummelhuis and Bakker (2012) present a typology of resources which combine two types of previously identified resources - key resources and macro resources. Key resources have been described as those resources that are responsible for the selection, alteration, or implementation of other resources (Thoits, 1994). These include resources such as personality traits, social power, or status. Macro resources, on the other hand, refer to "characteristics of the larger economic, social, and cultural system in which a person is embedded" (ten Brummelhuis \& Bakker, 2012, p. 548). This typology is arranged on two dimensions, that of source and transience. Sources of resources can be contextual or personal, while the transience of resources can be either 
volatile or structural. As described by Hobfoll (2002), contextual resources refer to those which are external to the individual. Examples include social support, marriage, or a home. Personal resources, however, are internal to the individual and include such resources as personality traits and energy. The second dimension of resource transience includes both volatile and structural resources. Volatile resources refer to those resources which can only be used once and for one purpose (e.g., time, physical energy), or which are fleeting in nature (e.g., mood). Structural resources, however, lie on the opposite spectrum, and refer to those resources which are of a more durable nature and can be drawn upon multiple times. Examples include a social network, home, skills, and mental resilience.

The Work-Home Resources Model extends COR theory to depict an explicit model of the work-to-home process (and conversely, the home-to-work process). In this model, work demands, resources, and outcomes are associated with personal resources, which are in turn associated with home outcomes, demands, and resources. These particular propositions are directly relevant to Study 1, in which workplace aggression, a workplace stressor, is found to be associated with psychological detachment, which can be assumed to generate individual resources. Lack of psychological detachment, in turn, is associated with increased levels of work-family conflict. Additionally, in Study 2, workplace aggression is conceptualized as a workplace demand which depletes employees of resources, which is seen in increases of perceived stress. This lack of personal resources in turn spills over to influence the nonwork domain, in the form of reduced parental warmth. 
Job Demands-Resources Model. The Job Demands-Resources Model posits that each occupation consists of both demands and resources. Job demands are "physical, psychological, social, or organizational aspects of the job that require sustained physical and/or psychological (cognitive and emotional) effort or skills and are therefore associated with certain physiological and/or psychological costs" (Demerouti \& Bakker, 2011, p. 2). Job resources, on the other hand, are also seen as being physical, psychological, social, or organizational job aspects that either serve a purpose in accomplishing work goals, reduce the impact of job demands, or generate growth, learning, and development.

The Job Demands-Resources Model suggests four main tenets (Demerouti \& Bakker, 2011). The first of which is that job demands lead to a health impairment process, whereby chronic demands diminish resources, leading to reduced health outcomes (e.g., increased burnout). Second, job resources contribute to a motivational process, such that employees with access to job resources experience higher levels of motivation and work engagement. Third, the buffering hypothesis suggests that the presence of job resources can diminish the negative influence of chronic job demands. Finally, the presence of job resources are said to be particularly influential on motivation and engagement in the face of high job demands (Bakker, Hakanen, Demerouti, \& Xanthopoulou, 2007). The JD-R Model shares similarities with COR theory as described above. Namely, both theories are grounded in the concept of resources. Unlike COR theory, however, much of the research on the JD-R Model to date focuses on the role of job-related demands and resources on work-related outcomes. 
Study 3 of this dissertation draws on both COR theory and the JD-R Model to explain both the depleting effects of workplace aggression as well as the proposed buffering effect of job-related resources (i.e., safety climate) in mitigating the negative association between workplace aggression and safety. Workplace aggression is conceptualized as a job demand that depletes employees of cognitive and emotional resources, leading to increased levels of burnout. Additionally, meta-analytic evidence has demonstrated that job demands are negatively associated with the absorption dimension of work engagement (Cole, Walter, Bedeian, \& O’Boyle, 2012). Relevant to the Study 3 of this document, workplace aggression may deplete individual resources necessary for engagement in safety behaviors (e.g., wearing personal protective equipment, following all relevant safety rules).

In addition to the main effects of workplace aggression on work outcomes, the current research also draws on the JD-R's proposition of the buffering effects of resources on job demands. In Study 3, two individual and organizational resources are proposed to attenuate the negative relationship between workplace aggression and safety outcomes. Mindfulness, a personal resource, in the presence of job-related demands may be particularly important for reducing this negative association. As Bakker and colleagues (2007) have noted, job-related resources are particularly salient in the face of high job demands. At the organization level, safety climate is proposed as a resource that may attenuate the relationship between resource loss associated with workplace aggression and safety outcomes. In the current context, the existence of job-related resources may contribute to a motivational process by which employees still engage in safety behaviors, even in the face of high levels of workplace aggression. 
Chapter 3: Shrugging it Off: Does Psychological Detachment from Work Mediate the Relationship between Workplace Aggression and Work-Family Conflict? ${ }^{1}$

Caitlin A. Demsky, Allison M. Ellis, and Charlotte Fritz

Portland State University

${ }^{1}$ Copyright $@ 2014$ by the American Psychological Association. Reproduced with permission. The official citation that should be used for referencing this material is: Demsky, C. A., Ellis, A. M., \& Fritz, C. (2014). Shrugging it off: Does psychological detachment mediate the relationship between workplace aggression and work-family conflict? Journal of Occupational Health Psychology, 19, 195-205. doi: $10.1037 / \mathrm{a} 0035448$ 


\section{Author Note}

Caitlin A. Demsky, Department of Psychology, Portland State University; Allison M. Ellis, Department of Psychology, Portland State University; Charlotte Fritz, Department of Psychology, Portland State University.

The writing of this manuscript was support by the National Science Foundation under Grant No. DGE-1057604. Any opinions, findings, and conclusions or recommendations expressed in this material are those of the authors and do not necessarily reflect the views of the National Science Foundation. The authors would like to thank Drs. Talya Bauer and Robert Wright for their thoughtful feedback on earlier drafts of this manuscript.

Correspondence concerning this article should be address to Caitlin A. Demsky, Department of Psychology, Portland State University, P.O. Box 751, Portland, OR, 97207-0751, USA. E-mail: cademsky@pdx.edu 


\begin{abstract}
The current study investigates workplace aggression and psychological detachment from work as possible antecedents of work-family conflict. We draw upon Conservation of Resources theory and the Effort-Recovery Model to argue that employees who fail to psychologically detach from stressful events in the workplace experience a relative lack of resources that is negatively associated with functioning in the nonwork domain. Further, we extend prior research on antecedents of work-family conflict by examining workplace aggression, a prevalent workplace stressor. Utilizing multi-source data (i.e., employee, significant other, and coworker reports), our findings indicate that selfreported psychological detachment mediates the relationship between coworker-reported workplace aggression and both self- and significant other-reported work-family conflict. Findings from the current study speak to the value of combining perspectives from research on recovery from work stress and the work-family interface, and point towards implications for research and practice.
\end{abstract}

Keywords: workplace aggression, recovery from work, psychological detachment, work-family conflict 
Shrugging it Off: Does Psychological Detachment Mediate the Relationship between Workplace Aggression and Work-Family Conflict?

Over the past fifty years, several demographic changes (e.g., an influx of women in the workplace, the aging of the workforce) have altered both the way employees work and the boundaries between home and work-life (e.g., flexibility of work hours, telecommuting; Hammer \& Zimmerman, 2011), signaling an increased need to examine the interface between work and nonwork. Work-family conflict (WFC), refers to "a form of interrole conflict in which the role pressures from the work and family domains are mutually incompatible in some respect" (Greenhaus \& Beutell, 1985, p. 77) and is associated with a number of important outcomes, including negative employee attitudes, and reduced performance, health, and well-being (Allen, Herst, Bruck, \& Sutton, 2000; Amstad, Meier, Fasel, Elfering, \& Semmer, 2011; Ford, Heinen, \& Langkamer, 2007). Given the potential for such deleterious effects, understanding the processes that drive WFC is critical for the well-being of employees and organizations alike.

Workplace aggression, a job stressor that may have serious implications for worklife balance, refers to "efforts by individuals to harm others with whom they work, or have worked, or the organizations in which they are currently, or were previously, employed" (Neuman \& Baron, 1997, p. 38). While traditional theoretical explanations of WFC (i.e., role theory; Kahn, Wolfe, Quinn, Snoek, \& Rosenthal, 1964) are useful for explaining how some work stressors (e.g., overtime) impede individuals from meeting demands in the nonwork domain (e.g., childcare responsibilities), these explanations are somewhat less intuitive when investigating relationships between psychosocial stressors, such as workplace aggression, and functioning in the nonwork domain. The current study 
argues that recovery from work stress (i.e., a process in which individuals are able to restore and rebuild resources lost due to prolonged exposure to work stressors; Sonnentag \& Fritz, 2007) represents a potential vehicle that can help explain the association between workplace aggression and WFC. No studies to-date have empirically examined this association, an important gap in the literature this study seeks to address.

Thus, this study contributes to past research in the following ways: First, workplace aggression has only recently begun to draw the attention of work-family researchers. This gap in the literature is surprising given the prevalence of aggression in the work context, as well as the serious employee and organizational outcomes associated with its occurrence (Bruk-Lee \& Spector, 2006; Hershcovis \& Barling, 2010; Hershcovis, 2011; Spector \& Jex, 1998).

Second, the current study integrates the work-family and recovery from work stress literatures to better understand one potential mechanism (i.e., psychological detachment) through which work stressors - in this case, workplace aggression — may be associated with higher levels of WFC. While recovery from work has been linked to a number of well-being indicators (e.g., sleep quality, vigor, burnout, general well-being; Fritz \& Sonnentag, 2005; Sonnentag, Binnewies, \& Mojza, 2008; Sonnentag \& Niessen, 2008), we know much less about the role of recovery as a mechanism through which work stressors relate to the nonwork domain.

Third, our study contributes to a growing body of literature examining the processes by which workplace stressors can transfer into the nonwork domain (Bolger, DeLongis, Kessler, \& Wethington, 1989). For example, work characteristics, such as low levels of support, have been associated with increased negative spillover (Grzywacz \& 
Marks, 2000). While a growing area of interest, previous studies of work-family spillover have rarely empirically identified the processes by which stressors or resources in the workplace are associated with the nonwork domain (e.g., Story \& Repetti, 2006; ten Brummelhuis \& Bakker, 2012). Accordingly, the current study aims to build on previous spillover research by illuminating potential mechanisms that underlie this process.

\section{Theoretical Background}

Work-family conflict has traditionally been viewed through the lens of role theory (Kahn et al., 1964). From this perspective, WFC arises when participation in one role makes it more difficult to engage in another role (Katz \& Kahn, 1978). Accordingly, stressors (e.g., workplace aggression) in the work domain are thought to impact WFC when addressing those stressors impedes one's ability to adequately address or fulfill one's role in the nonwork domain (e.g., increased rumination and lack of energy associated with psychological stress makes attending to a significant other's concerns at home more difficult). Inherent in this explanation is the idea that successful functioning in different roles requires the availability of resources (i.e., time, energy, affect). Building on this notion, we argue that it is useful to consider resource-based theoretical explanations employed in the research on recovery from work stress, particularly, conservation of resources theory (COR; Hobfoll, 1989) and the effort-recovery model (Meijman \& Mulder, 1998).

COR theory posits that individuals are motivated to maintain, build, and protect valued personal resources, which are defined as objects, conditions, personal characteristics, or energies that an individual values or that serve as a means for obtaining additional resources (Hobfoll, 1989). A key tenet of COR theory is that individuals 
experience stress when there is a threat of resource loss, an actual loss of resources, or a failure to regain resources after a loss. In the organizational setting, COR theory suggests that "work demands... are perceived as losses since meeting these demands requires the investment of valued resources" (Hobfoll \& Shirom, 2000, p. 69). On the other hand, opportunities to relax between stressors "allows for a regrouping of resources... and a chance to bolster resources for the future" (Hobfoll \& Shirom, 2000, p. 63). Building on this framework, research on recovery from work stress has argued that engaging in specific recovery experiences during nonwork time (e.g., psychological detachment), can both halt resource loss associated with work stressors, as well as build new resources (e.g., energy, positive mood), helping to restore depleted reserves (cf. Sonnentag \& Fritz, 2007).

While COR theory has been used to explain the relationship between threatened resources and individual stress, the effort-recovery model has been used to explain how recovery experiences can halt the process of resource loss (Meijman \& Mulder, 1998). Drawing on this model, Sonnentag and Fritz (2007) argued that recovery from work occurs when demands are no longer made on the same functional systems that are called upon during work. Psychological detachment, a recovery experience that allows employees to mentally remove themselves from work stressors and all work related demands, is one such process through which resources can be replenished. The presence of work stressors have been associated with decreased psychological detachment (e.g., Sonnentag \& Bayer, 2005). Specifically, when work stressors continue to impact employees outside of the work domain, employees are less likely to psychologically detach from work, leading to a failure to replenish resources that were lost during the 
workday. This lack of replenishment in turn may negatively impact the nonwork domain, as individuals have insufficient resources with which to approach nonwork-related tasks and interactions (i.e., fulfill nonwork roles).

We argue that role theory on the one hand and COR and the effort-recovery model on the other hand complement one another. Specifically, while role theory focuses on the demands and expectations of particular roles, COR theory and the effort-recovery model utilize a resource-based perspective to explain the relationship between work and nonwork domains. Utilizing both perspectives, we seek to examine the relationship between workplace aggression and WFC, as well as explore psychological detachment from work as an underlying mechanism of that relationship.

\section{Workplace Aggression}

Past research indicates that aggression at work is a frequently experienced work stressor, that, when encountered, typically elicits feelings of anger, annoyance, and frustration (Keenan \& Newton, 1985; Narayanan, Menon, \& Spector, 1999; Nixon, Mazzola, Bauer, Krueger, \& Spector, 2011). Prevalence statistics suggest that nearly $41 \%$ of U.S. employees report having experienced psychological aggression (i.e., nonphysical forms of aggression) at work over the last 12 months (Schat, Frone, \& Kelloway, 2006). A recent meta-analysis found that workplace aggression was associated with a number of negative health outcomes (e.g., sleep disturbances, backaches, headaches, fatigue, alcohol use and gastrointestinal problems; Frone, 1999; Nixon et al., 2011). Thus, continued investigation of the construct remains an important avenue for occupational health research. 
A number of similar, overlapping constructs have proliferated within the workplace aggression literature over the past 15 years. For instance, constructs examined under this umbrella term include workplace bullying (Einarsen, 1999), mobbing (Leymann, 1990), and harassment (Brodsky, 1976). Hershcovis (2011) noted conceptual and measurement overlap among constructs and demonstrated similar patterns of relationships between five forms of workplace aggression (i.e., incivility, abusive supervision, bullying, social undermining, and interpersonal conflict) and relevant employee outcomes. She recommended future research utilize a general perspective on workplace aggression. Hence, we refer here to the general construct of workplace aggression, but, where relevant, review literature pertaining to more specific constructs (e.g., incivility, interpersonal conflict).

\section{Workplace Aggression and WFC}

In the current study, we argue that increases in coworker-reported workplace aggression are associated with increases in $\mathrm{WFC}^{2}$. Role theory proposes that a lack of energy may be one source of difficulty in participating in multiple roles. In the context of the current study, resources consumed through experiences of workplace aggression may contribute to increased difficulties in managing the nonwork domain (i.e., higher levels of WFC). For example, and in line with COR theory, resources depleted through the experience of workplace

\footnotetext{
${ }^{2}$ WFC has been identified as bi-directional, such that work stressors can interfere with family demands, and vice versa (Greenhaus \& Beutell, 1985). Although our primary theoretical interest is with the work-tofamily direction of WFC, we included an assessment of family-to-work conflict and conducted additional analyses in order to rule out the possibility that the latter construct was driving the observed relationships.
} 
aggression (e.g., cognitive, emotional) are then unavailable for investment in the nonwork domain.

Accordingly, research suggests work stress can impact functioning in the nonwork domain (e.g., increased marital withdrawal and anger; Brodsky 1976; Repetti, 1989; Saxbe, Repetti, \& Graetsch, 2011; Story \& Repetti, 2006). More specifically, some research has begun to examine the relationship between various forms of workplace aggression and WFC (e.g., Carlson, Ferguson, Hunter, \& Whitten, 2012; Hoobler \& Brass, 2006; Lim \& Lee, 2011; Restubog, Scott, and Zagenczyk, 2011; Tepper, 2000). For example, daily job stressors - including negative social interactions - have been associated with increases in marital withdrawal and marital anger (Story \& Repetti, 2006). Restubog et al. (2011) found that abusive supervision was associated with increased spouse undermining, and that this relationship was mediated via increased psychological distress. Finally, a more recent examination of abusive supervision found that its effects on WFC and FWC were transmitted through surface acting and burnout (Carlson et al., 2012). Together, these studies point to potential associations between workplace aggression and WFC. Accordingly, we propose that individuals who experience higher levels of workplace aggression will also experience higher levels of WFC.

H1. Workplace aggression will be positively related to WFC.

\section{The Role of Psychological Detachment}

Psychological detachment from work has been defined as one's "sense of being away from the work environment" (Etzion, Eden, \& Lapidot, 1998, p. 579). 
In addition to refraining from taking work-related calls or answering work-related emails, psychological detachment also implies a mental disengagement from work during nonwork time (Sonnentag \& Fritz, 2007). In line with COR theory, the experience of workplace aggression depletes an individual's resources (e.g., emotional, cognitive). In turn, prolonged or repeated exposure to workplace aggression may result in strain reactions in the absence of recovery from work, as suggested by the effort-recovery model. Extended activation in the form of affective and cognitive reactions to instances of workplace aggression may make it more difficult for employees to mentally disengage from work and engage in recovery experiences during nonwork time. In the context of the current study, experiencing workplace aggression may make it more difficult to engage in psychological detachment after work, which may lead to a lack of resource replenishment. Drawing on role theory, one can argue that failing to psychologically detach from work may make it more difficult to transition between roles, increasing the amount of experienced interrole conflict.

Prior research indicates that stressors at work can impede employees' psychological detachment outside of work. For example, increased workload and emotional dissonance are negatively related to psychological detachment (Sonnentag \& Bayer, 2005; Sonnentag, Kuttler, \& Fritz, 2010). One recent study showed that social conflicts with customers were also negatively associated with employees' psychological detachment and that this relationship was mediated by increased negative affect (Volmer, Binnewies, Sonnentag, \& Niessen, 2012). Similarly, experiencing workplace aggression is a stressor that arguably draws upon a number of individual resources (e.g., emotional, 
cognitive), and has been related to increases in negative emotions (e.g., anger, frustration) and subsequent rumination (Keenan \& Newton, 1985). It is important to note that a failure to psychologically detach is distinct from rumination (e.g., Donahue et al., 2012). A lack of psychological detachment due to workplace aggression may consist of any one of several experiences, including ruminating about the conflict, venting to family members or friends, engaging in conflict resolution, or catching up on work due to time lost to managing conflicts. Thus, it is likely that employees will have difficulty mentally distancing themselves from experiences of workplace aggression when they leave work. For instance, employees may have difficulty detaching from negative workplace interactions and instead may be left wondering how to resolve them, or assessing the impact the conflict may have on future interactions, workplace dynamics, or their own performance levels. Therefore, we argue that workplace aggression will be negatively related to psychological detachment from work.

\section{H2. Workplace aggression will be negatively related to psychological} detachment from work during nonwork time.

Based on the effort-recovery model (Meijman \& Mulder, 1998), psychological detachment provides an opportunity to halt work-related resource loss and promote resource replenishment. These resources, in turn, should be available once again for use in the nonwork domain, allowing employees to meet nonwork demands. Conversely, a preoccupation with work stressors (i.e., workplace aggression) may be associated with increased distraction or negative emotions at home, which may increase the likelihood of WFC. Furthermore, failing to psychologically detach from work may increase employees' perceptions of their work interfering with their home life. For example, time 
spent worrying about one's work may detract from time that could otherwise be spent with a significant other, children, or friends. Employees who are able to psychologically detach upon leaving the workplace may be more likely to reduce negative emotions and thoughts associated with workplace stressors, including workplace aggression.

While a growing body of research demonstrates positive associations between psychological detachment and a number of nonwork outcomes (e.g., well-being and performance; Fritz, Yankelevich, Zarubin, \& Barger, 2010; Sonnentag, 2012), few studies so far have linked the experience of psychological detachment to reduced WFC. One recent study found that work-interfering-with-home behaviors such as worrying about work and doing work at home, akin to low psychological detachment, were positively related to higher partner reports of WFC (Green, Schaefer, MacDermid, \& Weiss, 2011). Additionally, daily psychological detachment from work has been associated with lower levels of home-to-work interference for those with low work role salience (Sanz-Vergel, Demerouti, Bakker, \& Moreno-Jimenez, 2011). Finally, research has found that psychological detachment is negatively associated with WFC and moderates the relationship between WFC and psychological strain (Moreno-Jimenez et al., 2009). Thus, we propose that higher levels of psychological detachment from work will be associated with lower levels of WFC.

H3. Psychological detachment from work during nonwork time will be negatively related to WFC.

So far, we have provided arguments for the relationships between workplace aggression and WFC, workplace aggression and psychological detachment, and psychological detachment and WFC. In addition, we propose psychological detachment 
as a mechanism that may explain the relationship between workplace aggression and WFC. Specifically, we hypothesize that experienced workplace aggression will be associated with a lowered ability to psychologically detach from work. In line with the effort-recovery model, when employees fail to psychologically detach from work, they will fail to halt the resource loss initiated by workplace aggression. This resource deficit will in turn impact the nonwork domain, which, we propose, manifests in higher levels of WFC. A model of these proposed relationships can be seen in Figure 3.1. Thus, we hypothesize the following:

H4. The effects of workplace aggression on WFC will be mediated by psychological detachment from work during nonwork time. 


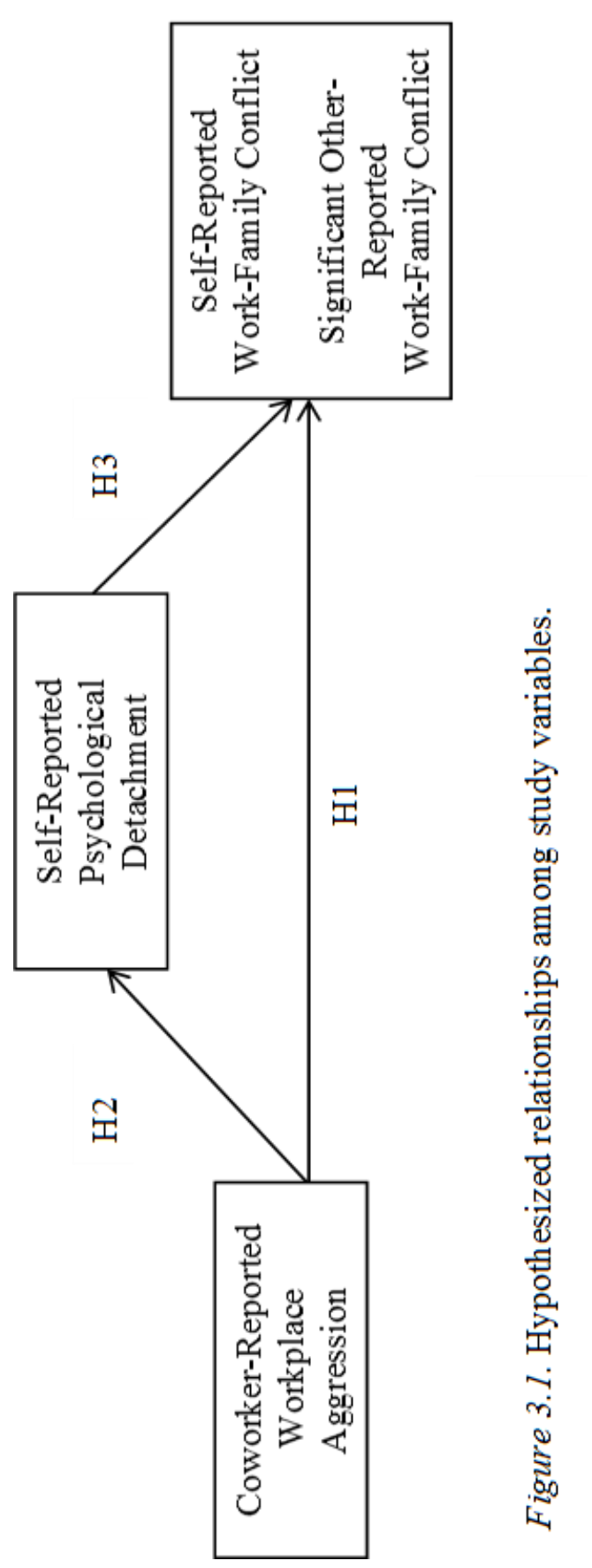




\section{Method}

\section{Procedure}

Participants were recruited from several U.S. colleges and universities. Once schools agreed to participate in the study, recruitment e-mails were sent to potential participants. Survey packets were sent out to 299 non-academic employees and included an introduction letter and survey materials.

For the current study, self-reported, coworker-reported, and significant otherreported data was collected to examine the research hypotheses. The target employee reported information on their experience of psychological detachment and WFC in reference to the past few weeks. At the same time, the target employee was asked to choose one coworker who knew his or her work well and ask this coworker to fill out a survey pertaining to the target employee's work environment (including experienced workplace aggression). In addition, the target employee was asked to have a significant other or partner fill out a survey pertaining to the target employees' nonwork domain (including WFC).

Data were collected from multiple sources (i.e., target employee, coworker, and significant other) to address potential issues regarding common method bias (Podsakoff, MacKenzie, Lee, \& Podsakoff, 2003). Common method variance can have a sizeable effect on observed relationships between constructs, potentially artificially inflating the relationship between key variables. Podsakoff et al. (2003) suggest several procedural remedies for addressing the issue of common method biases, one of which is to obtain measures of the predictor and criterion variables from different sources, an approach we 
adopted for the current study. While the majority of empirical research on workplace aggression focuses on the victim's perceptions of aggression, several studies have demonstrated that others in the workplace can reliably report on the frequency of coworkers' experienced aggression (Bruk-Lee \& Spector, 2006; Penney \& Spector, 2005). Additionally, utilizing significant other reports of WFC contributes to more recent work in the work-family domain, which has called for the inclusion of significant otherreports of WFC (e.g., Casper, Eby, Bordeau, Lockwood, \& Lambert, 2007; Green et al., 2011).

\section{Participants}

Of the 299 participants who were solicited for participation, 173 surveys (58\% response rate) were completed and returned. Sixty-six of these were left out of the analyses due to missing data or missing other reports (i.e., coworker or significant other surveys). The final 107 participants consisted of 91 women (85\%) and 16 men (15\%). The average age of the participants was 44.65 years $(S D=10.71)$. Mean job tenure was 10.02 years $(S D=8.96)$ and $45 \%$ of the participants held supervisory positions. Some of the jobs included in the sample were administrative assistant, coordinator of programs, director, web developer, and library associate. Regarding educational level, 39\% were college graduates, 30\% held a master's degree, and 5\% had earned a doctoral degree.

Nonresponse analyses comparing employees whose data were removed due to missing coworker or significant other reports to those who returned all three reports (i.e., self, significant other, and coworker) using independent-samples $t$ tests and chi-square tests showed that the two groups did not differ significantly on any demographic variables (i.e., age, gender, marital status, living with children). Employees with missing 
data were significantly higher in self-reported WFC $(M=3.59, S D=1.74)$ and selfreported FWC $(M=2.53, S D=1.37)$ than those employees who returned all three surveys (WFC: $M=3.04, S D=1.64$; FWC: $M=1.99, S D=1.18), t(171)=-2.06, p=.04$, $t(171)=-2.77, p=.01$, respectively). No significant differences were found between the groups regarding workplace aggression $t(123)=-.25, n s$, or psychological detachment $t(171)=1.04, n s$. A chi-square test showed no significant differences in gender $\chi^{2}(1 ; N=$ $173)=.02, n s$, marital status $\chi^{2}(1 ; N=172)=.01, n s$, or living with children $\chi^{2}(1 ; N=$ $172)=.01, n s$ between both groups.

Coworkers reporting on target employees included 82 women (77\%) and 23 men $(22 \%)$. The average age of coworkers was 43.63 years $(S D=10.54)$. On average, coworkers had worked a total of 10.65 years $(S D=8.59)$ in their current position, and had worked with the target employee for an average of 5.32 years $(S D=5.26)$.

Significant others included 34 women (32\%) and 71 men (68\%). On average, significant others were 43.95 years old $(S D=10.92)$ and had known the target employee for 19.18 years $(S D=13.20)$.

\section{Measures}

\section{Self-Reports}

Psychological detachment. We used four items from the Recovery Experiences Questionnaire (Sonnentag \& Fritz, 2007) to measure target employees' level of psychological detachment during nonwork time. Response options were given on a 5point scale $(1=$ not at all to $5=$ always $)$. A sample item was, "During nonwork time, I didn't think about work at all.” Cronbach's alpha was .84. 
Work-family conflict. WFC was assessed with a five-item scale (Netemeyer, Boles, \& McMurrian, 1996). Responses were given on a 7-point scale ( 1 = strongly disagree to $7=$ strongly agree). The target employees and significant others were asked to rate the target employees' level of WFC over the past few weeks. A sample item was, "The demands of my work interfered with my home and family life." Cronbach's alpha was .92 and .91 for self-reported and significant other-reported WFC, respectively.

Given the bi-directional nature of conflict (Edwards \& Rothbard, 2000;

Greenhaus \& Beutell, 1985), and in order to allow for tests of reverse relationships, we assessed family-work conflict (FWC) in addition to WFC. As with WFC, FWC was measured with the five-item scale by Netemeyer et al. (1996). A sample item included, "I had to put off doing things at work because of demands on my time at home" (self-report $\alpha=.90$, significant other-report $\alpha=.85$ ).

\section{Coworker-Reports}

Workplace aggression. Three items from the Interpersonal Conflict at Work Scale (ICAWS; Spector \& Jex, 1998) were administered to target employees' coworkers to measure the target employee's level of experienced workplace aggression. Items included, "How often did other people yell at him/her at work?", "How often were people rude to him/her at work?", and "How often did other people do nasty things to him/her at work?" We chose to omit one item from the original scale ("How often did you get into arguments with others at work?") due to wording that may blur the distinction between the target employee as a perpetrator or victim of workplace aggression. All items were measured on a 5 -point Likert scale $(1=$ rarely to $5=$ very often $)$. Cronbach's alpha for coworker reports of this three-item scale achieved acceptable levels of reliability at .78. 


\section{Control Variables}

Demographic variables. We controlled for age, gender, marital status, and whether or not the participants were living with children. Control variables were chosen based on findings from previous research indicating potential relationships with key study variables (Byron, 2005; Hershcovis et al., 2007). For example, as employees age, they are more likely to have a greater number of family roles to juggle (e.g., childcare, eldercare), and in turn experience greater WFC. Gender has also been a focus of past WFC research, suggesting that females tend to report higher levels of WFC and experience a more negative impact on the quality of work life due to conflict between work and home (Duxbury \& Higgins, 1991). Finally, employees who are married and living with children are also expected to experience more frequent conflict between work and nonwork domains (Byron, 2005).

\section{Results}

\section{Preliminary Analysis}

Means, standard deviations, variable intercorrelations and alpha coefficients are presented in Table 3.1. The key study variables were all related to one another in the expected directions. Specifically, workplace aggression was associated with lower levels of psychological detachment $(r=-.34, p<.001)$ and higher levels of self- and significant other-reported WFC $(r=.29, p<.01 ; r=.33, p<.01$, respectively). Psychological detachment was, in turn, associated with lower levels of both self- and significant otherreported WFC $(r=-.51, p<.001 ; r=-.44, p<.001$, respectively $)$. 


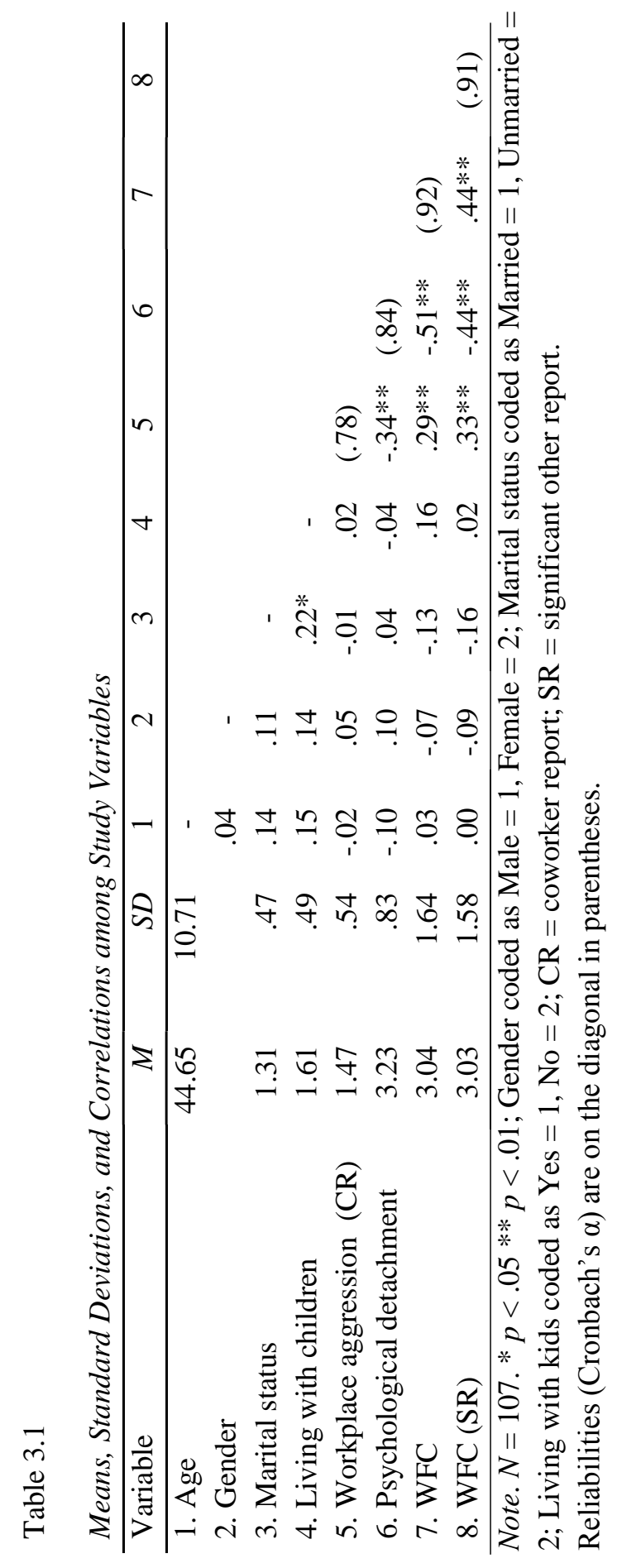




\section{Hypothesis Testing}

Hypothesis 1 proposed that workplace aggression would be positively related to WFC. To test Hypothesis 1 , we entered control variables ${ }^{3}$ (i.e., age, gender, marital status, living with children) in the first step of the regression equation followed by the independent variable (i.e., workplace aggression) in the second step. Hierarchical regression analyses showed that coworker-reported workplace aggression ${ }^{4}$ was significantly positively related to both self-reported and significant other-reported WFC $(\beta=.29, t(104)=3.10, p=.003 ; \beta=.31, t(103)=3.28, p=.001)$, providing full support for Hypothesis 1 (see Table 3.2). Specifically, higher levels of workplace aggression were associated with higher levels of both self-reported and significant other-reported WFC.

Hypothesis 2 stated that workplace aggression would be negatively related to psychological detachment during nonwork time. To test Hypothesis 2, we entered control variables (i.e., age, gender, marital status, living with children) in the first step of the regression equation followed by the independent variable (i.e., workplace aggression) in the second step. Results show that coworker-reported workplace aggression was significantly negatively related to self-reported psychological detachment $(\beta=-.36$, $\left.t(104)=-3.86, p<.001, \Delta R^{2}=.10, \Delta F=11.46\right)$, supporting Hypothesis 2. Specifically, higher levels of workplace aggression were associated with lower levels of psychological detachment during nonwork time.

\footnotetext{
${ }^{3}$ Controlling for negative affectivity did not substantially change the results reported in-text. These results can be obtained from the first author upon request.

${ }^{4}$ A similar pattern of results were found after cross-validating our proposed model using self-reports of workplace aggression. These results can be obtained from the first author upon request.
} 
Hypothesis 3 predicted that psychological detachment from work would be negatively related to WFC. Results from these hierarchical regression analyses (see Table 3.2) showed that self-reported psychological detachment was significantly negatively associated with both self-reported WFC and significant other-reported WFC $(\beta=-.45$, $t(104)=-4.94, p<.001 ; \beta=-.37, t(103)=-3.92, p<.001)$, providing full support for Hypothesis 3. Specifically, higher levels of psychological detachment were associated with lower levels of both self and significant other-rated WFC. 


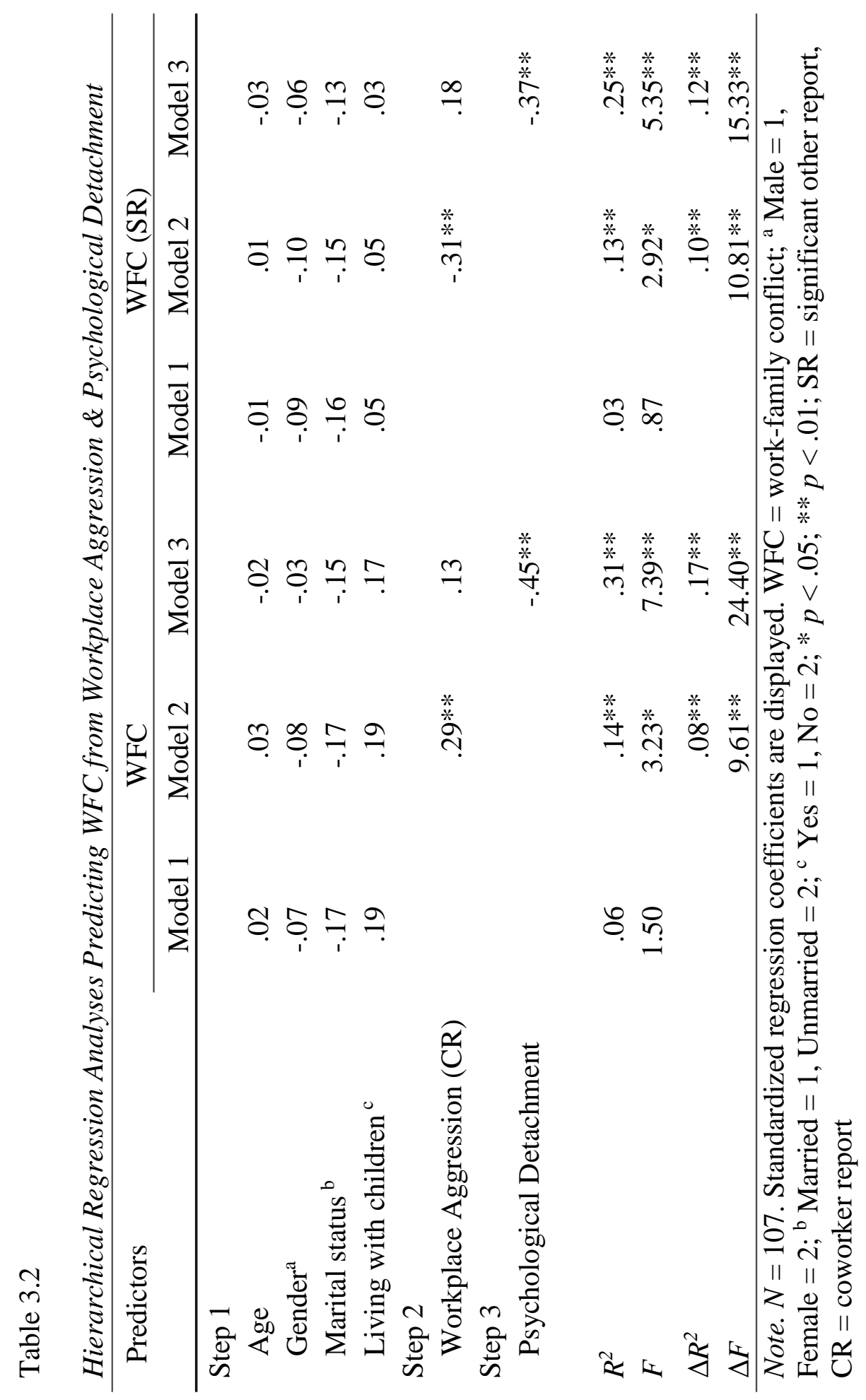


Hypothesis 4 proposed that psychological detachment would mediate the relationship between workplace aggression and WFC. We tested this hypothesis using the bias-corrected bootstrapped mediation tests recommended by Preacher and Hayes (2004, 2008). This test has been recommended as a more accurate test of mediation (i.e., the Sobel test), particularly in the case of smaller sample sizes (Shrout \& Bolger, 2002). Additionally, this test does not assume a normal sampling distribution of indirect effects (MacKinnon, Lockwood, \& Williams, 2004; Preacher \& Hayes, 2008; Shrout \& Bolger, 2002). We conducted Preacher and Hayes' (2008) approach using the INDIRECT macro for SPSS to request 5,000 bootstrapped resamples from the obtained data. We also derived the $95 \%$ bias-corrected confidence intervals (CIs). The mediation effect is considered statistically significant when the upper and lower bound of the corrected CIs do not contain zero.

As seen in Table 3.3, the mediation tests following Preacher and Hayes' (2008) guidelines provided support for Hypothesis 4. The bootstrapped 95\% CIs do not contain zero, confirming the presence of statistically significant indirect effects of coworkerreported workplace aggression on self- and significant other-reports of WFC via selfreported psychological detachment. 


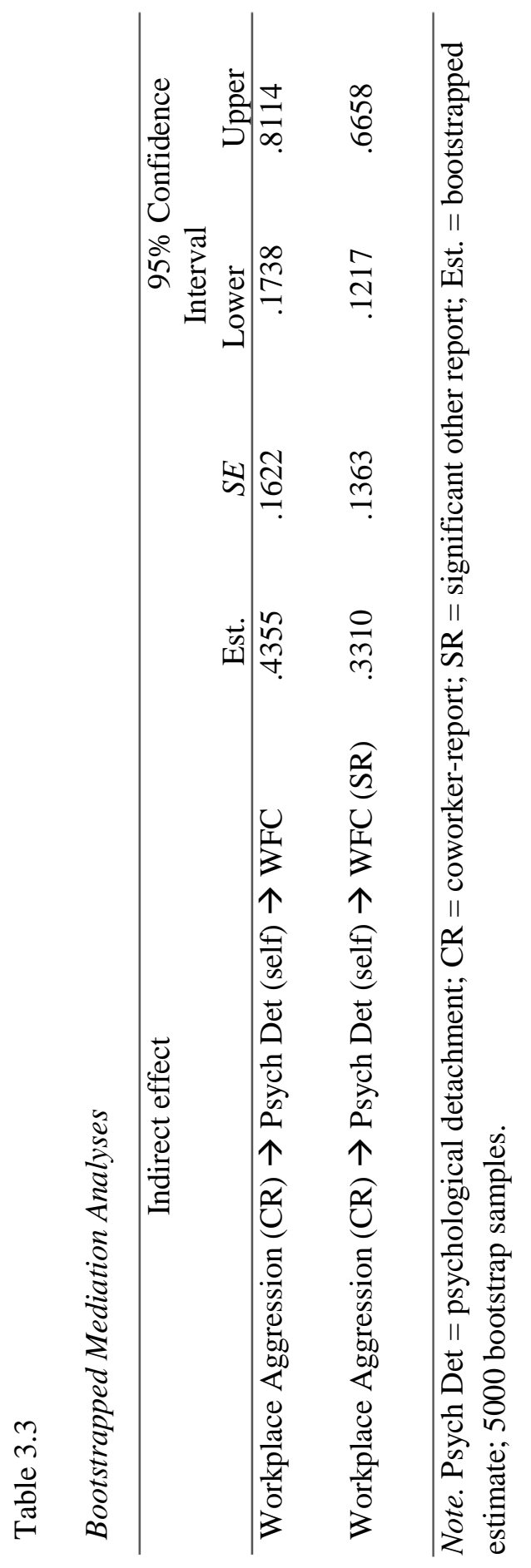




\section{Additional Analyses}

As WFC has been defined as a bi-directional construct (Edwards \& Rothbard, 2000; Greenhaus \& Beutell, 1985), work-family researchers often collect and analyze data on both WFC and family-to-work conflict (FWC). To rule out alternative explanations, in which experiencing FWC may be associated with higher levels of workplace aggression, we tested FWC as a predictor of workplace aggression, controlling for age, gender, marital status, and living with children.

Accounting for control variables, self-reported FWC conflict was associated with increased coworker-reported aggression $(\beta=.25, t(104)=2.36, p=.02)$, though significant other-reported FWC was not significantly related to coworker-reported workplace aggression $(\beta=.16, t(103)=1.49, n s)$. Further, bootstrapping results indicated a weak significant indirect effect of self-reported FWC on coworker-reported workplace aggression via psychological detachment $($ Est. $=.0537, S E=.0243$, CI Lower $=.0138, \mathrm{CI}$ Upper $=.1128$ ). These results are consistent with previous work-family research, which suggests that work stressors are associated with higher levels of both WFC and FWC, though relationships with FWC are typically weaker (Byron, 2005).

\section{Discussion}

The results of our study indicate positive relationships between workplace aggression and both self- and significant other-reported WFC, such that as employees experience more workplace aggression, WFC increases. This finding is in line with past research indicating that specific stressors in the work context can be associated with higher WFC (Byron, 2005; Michel et al., 2011). Furthermore, our results suggest that employees who experience higher levels of workplace aggression are less likely to 
psychologically detach from work, and possibly, from the very conflicts themselves.

These findings support recent research by Volmer and colleagues (2012) that showed that social conflicts with customers were negatively related to psychological detachment.

Finally, our results indicate that psychological detachment acts as a mediator of the relationship between workplace aggression and WFC.

\section{Potential Limitations}

As with any study, there are limitations of the current research that should be addressed in future studies. First, our data were collected at a single point in time which introduces the potential for inflation of correlations among the study variables, as well as making inferences of causality inappropriate. However, we attempted to reduce concerns related to common method bias (Podsakoff et al., 2003) by collecting both self- and other-reports of key study variables. For example, due to the observable nature of workplace aggression (Porath \& Pearson, 2010), we collected co-worker reports of workplace aggression. Furthermore, both self and significant other reports of WFC were obtained, making it possible to compare our results across sources. Findings were consistent across both reports, providing increased confidence that the results obtained were meaningful. Additionally, in order to address potential alternative explanations for our results, we conducted additional analyses to test the relationship between FWC and workplace aggression. While our findings generally support our theoretical assumption that workplace factors are associated with outcomes in the family domain, we do acknowledge that the pathways may be bi-directional, and are not the first to find evidence for such relationships (e.g., Byron, 2005). Future research should explore the strengths and directionality of these pathways in more detail. 
Second, there may be limitations with regard to the generalizability of the current findings. Our study was conducted within a single industry, and was primarily comprised of female participants; however, given the prevalence of workplace aggression and WFC across industries and organizations (Frone, Russell, \& Cooper, 1992; Fujishirio, Gee, \& de Castro, 2011; Quine, 1999; Schat et al., 2006), we expect that these are important and relevant constructs despite the organizational context. Furthermore, it is worth noting the results of our nonresponse analyses, which indicated significant differences between selfreports of WFC and FWC, such that those individuals who returned all three sources of data (i.e., self, significant other, and coworker) reported lower levels of WFC and FWC than those who were removed from the dataset due to missing significant other or coworker reports. As such, our findings may not fully reflect those individuals with higher levels of WFC and FWC. It may be that at high levels of WFC and FWC, additional mechanisms (e.g., social support, coping styles) serve to explain the relationship between work stressors (e.g., workplace aggression) and WFC.

\section{Implications for Research}

Our findings contribute to prior research in a number of ways. First, they provide evidence for one mechanism (i.e., lack of psychological detachment) through which workplace aggression may negatively impact the nonwork domain. While correlational evidence supports the relationship between stressors in the workplace and nonwork constructs like WFC (e.g., Byron, 2005; Ford et al., 2007; Michel et al., 2011), little research has been devoted to understanding the underlying mechanisms that explain these associations. Our findings demonstrate the utility of integrating the literatures on 
recovery from work stress and work-family dynamics in order to illuminate these potential mechanisms.

For example, while WFC is often explained through the lens of role theory, the results of the current study demonstrate the utility of more resource-based perspectives commonly employed in the recovery from work stress literature. Nevertheless, it will be important for future research to employ time-lagged studies that separate measurement of focal variables and allow for the direct comparison of alternative models in order to support such theoretical assumptions. For example, designs that are able to assess variation in reported workplace aggression, psychological detachment, and WFC on a daily basis can provide valuable insight into the relationships among these constructs.

Second, in examining differential outcomes it will be important for future research to consider the source of the conflict. Prior research indicates that aggression experienced by different sources, either within or outside the organization, may impact how, and to what extent, aggression negatively affects employee outcomes (Hershcovis $\&$ Barling, 2010). Conversely, the primary research question in the current study concerned whether or not aggression - as an overall stressor-would interfere with employees' ability to psychologically detach from work and in turn, their ability to meet nonwork demands. We assumed that while the source of aggression may provide information with regard to the intensity of the stressor (and thereby the magnitude of the relationship between aggression and WFC), the mechanisms through which workplace aggression impacts WFC would remain constant (i.e., a failure to psychologically detach from work-related thoughts and demands). However, extending this work to examine potential differences in the severity of experienced WFC as a result of different sources 
of workplace aggression would be a logical next step. Moreover, as future research examines alternative mediating mechanisms, beyond psychological detachment, and additional outcomes, it will be important to investigate whether the source of workplace aggression affects the manifestation of these relationships.

Finally, our study is one of the first to examine workplace aggression as it relates to experiences in the nonwork domain. Recent reports indicate that workplace aggression, in varying forms, is experienced by as many as $96 \%$ of workers (Porath \& Pearson, 2010), and is acknowledged as a prevalent workplace stressor (Herschovis \& Barling, 2010). Nevertheless, workplace aggression has received very little attention in terms of its potential impact on nonwork outcomes. Our study builds on recent findings (e.g., Moreno-Jimenez et al., 2009; Volmer et al., 2012) by examining further outcomes in the nonwork domain; namely, the indirect effects of workplace aggression on WFC. In examining psychological detachment as a potential mediator of these indirect effects, our study contributes to the growing body of literature on positive and negative spillover (Bolger et al, 1989; Grzywacz \& Marks, 2002). Extending our understanding of how the experience of workplace aggression impacts individual employees as they transition to their lives away from work is an important contribution of the current study and a building block for future occupational health research.

\section{Practical Implications}

Our study indicates that psychologically detaching from work may be most important when it is also the most difficult (i.e., on days that have been particularly stressful or emotionally charged). Previous research indicates workplace aggression is associated with a host of negative health-related outcomes for employees, including 
emotional exhaustion and impaired physical well-being (Hershcovis \& Barling, 2010). As individuals transition from the work to the home domain, they are likely to carry these strains with them (Edwards \& Rothbard, 2000), creating a downward spiral that is perpetuated by demands in both domains. The results of our study suggest that at least a portion of this process may occur through a failure to psychologically detach from the work domain. This has important implications for individual interventions. Our results suggest that employees who fail to detach themselves from thoughts and activities related to negative experiences at work may continue to experience the negative effects of workplace aggression, extending into the nonwork domain. Employees can be trained on the benefits of psychological detachment, and utilize goal-setting techniques to focus on segmenting their work and nonwork lives (e.g., Hahn, Binnewies, Sonnentag, \& Mojza, 2011). Furthermore, previous research has identified a host of beneficial effects that occur as a result of psychological detachment (e.g., increased positive mood, decreased exhaustion; Sonnentag et al., 2008; Sonnentag, Binnewies, \& Mojza, 2010), meaning that a reduction in WFC is only one of many potential positive effects of this psychological experience.

Kossek, Lewis, and Hammer (2010) argued that as changing technologies make 24/7 work possible, organizations may need to focus less attention on helping employees integrate their work and home domains, and more attention on helping them segment the two. While we did not examine the moderating effects of psychological detachment, previous research (e.g., Moreno-Jimenez et al., 2009; Sonnentag \& Bayer, 2005) has found that psychologically detaching from work can buffer the negative effects of stressors on employee outcomes. Taking this into account, in conjunction with our 
findings, managers can play a more active role in making sure their employees have adequate opportunities for psychological detachment from work. For example, creating a norm of segmentation (Edwards \& Rothbard, 2000) within the work group by limiting communications during nonwork time is a signal to employees that they can fully transition from work to home during their time away from work (Park, Fritz, \& Jex, 2011). Therefore, employers should encourage their employees to detach from work by creating norms and/or policies related to nonwork time, thereby enabling employees to appropriately allocate their resources outside of work.

Perhaps more importantly, these findings speak to the need for organizations to take measures to effectively manage and mitigate aggression in the workplace. Building on prior research which has suggested a relationship between workplace aggression and impaired functioning and well-being in the work context (e.g., Hershcovis \& Barling, 2010), our findings suggest that aggression is also negatively associated with functioning in the nonwork context. Thus, it behooves the responsible employer to take steps to prevent workplace aggression before it occurs; previous discussions surrounding the prevention of incivility have been presented by Pearson and Porath (2005) and are similarly applicable to this discussion. For instance, these authors suggest managers set zero-tolerance policies related to aggression and be aware of potential problem employees in order to address concerns head on. Herschovis and Barling (2006) point to the necessity of eliminating situational predictors of aggression and creating transparent and non-threatening work environments in order to prevent aggressive acts. It is also critical that employers react supportively to employees who report being victims of workplace aggression, and take complaints of such acts seriously (e.g., Duffy \& Sperry, 
2007, Lewis \& Orford, 2005). Other research suggests employees’ perceptions of organizational policies and norms related to workplace aggression can be important deterrents for those inclined to engage in aggressive acts (Dupre \& Barling, 2006). Thus, a primary implication of the current research is that organizations should take measures to prevent workplace aggression before it occurs.

\section{Conclusion}

In the current study, we hypothesized, and found, full support for psychological detachment as a mediator of the relationship between coworker-reported workplace aggression and WFC. Our findings generalized across both self- and significant otherreports of WFC, suggesting that psychological detachment may be a theoretically and practically important mechanism to explain how work stressors negatively impact engagement in nonwork roles. In addition to contributing to both the work-family and recovery from work stress literatures, our study offers several important practical implications, including suggestions for the development of individual and organizational interventions, as well as for management practices that help reduce aggression in the workplace and are supportive of employee psychological detachment during nonwork time. 
Chapter 4: Extending the Examination of Nonwork Outcomes of Workplace Aggression:

\section{A Longitudinal Examination}

The results of the first study of this dissertation indicate that workplace aggression may spillover to the nonwork domain in the form of increased work-family conflict via decreased psychological detachment from work during nonwork time. This first study relied on multi-source cross-sectional reports to examine the first of three general research questions posed by this dissertation: Does psychological detachment from work serve as a potential mechanism through which workplace aggression is associated with increased work-family conflict? While the results offer several contributions to the literature on workplace aggression and work-family conflict, the findings also introduce several additional questions for examination. For example, though workplace aggression may spill over to the nonwork domain to influence the employee and their significant other (e.g., Demsky, Ellis, \& Fritz, 2014; Green, Schaefer, MacDermid, \& Weiss, 2011), it may also be possible that experiences of workplace aggression may influence other members of the family, including children of employees. In order to overcome some of the limitations posed by the first enclosed empirical study (e.g., cross-sectional design), the following study was proposed. The hypothesized relationships can be seen in Figure 4.1. 


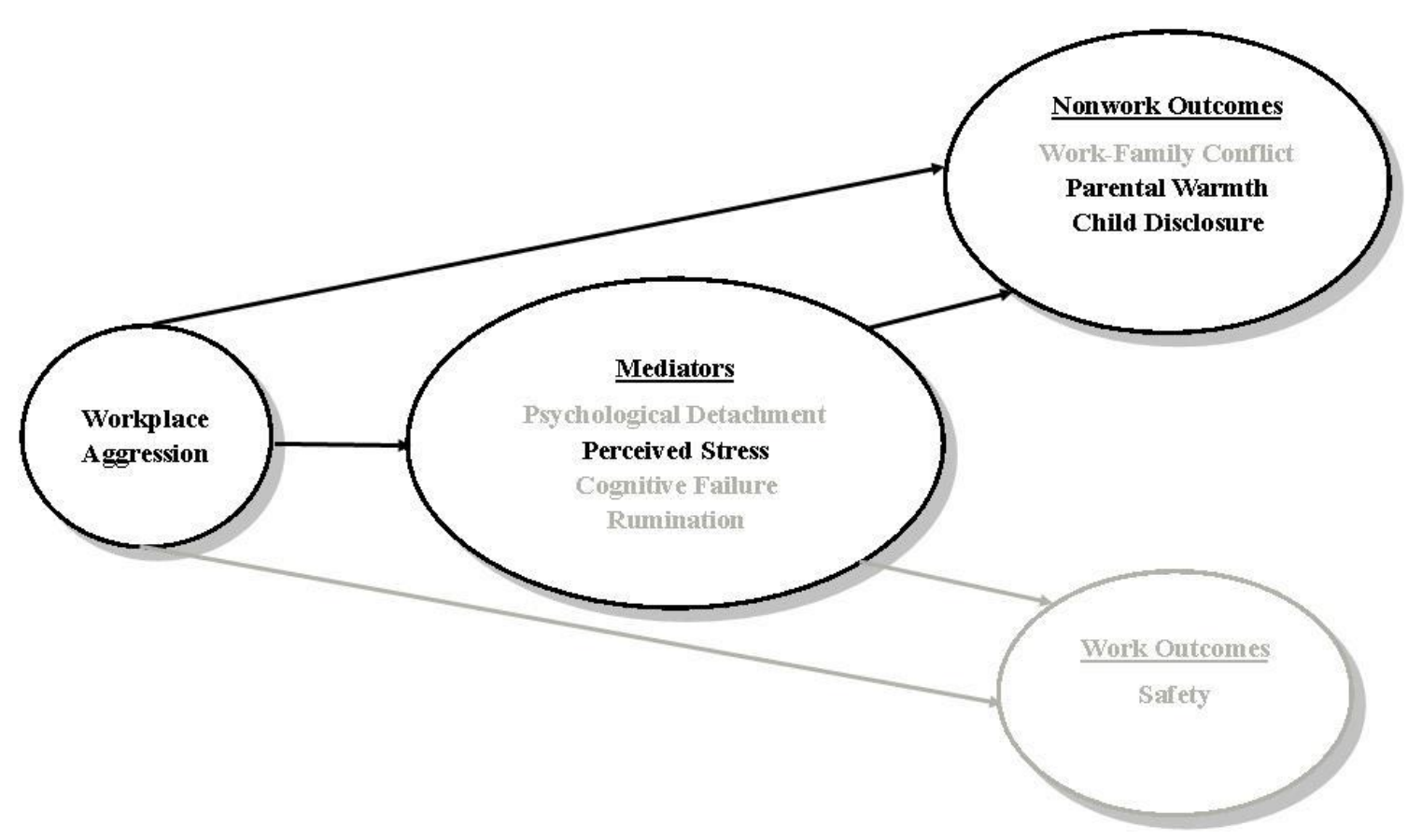

Figure 4.1 Summary model of the proposed relationships examined in Study 2. 
Chapter 5: The Role of Perceived Stress in the Relationship between Workplace Aggression and Parenting: Evidence from the Work, Family, \& Health Network

Caitlin A. Demsky Leslie B. Hammer

Portland State University

(in preparation for submission to Human Relations) 


\section{Author Note}

Caitlin A. Demsky, Department of Psychology, Portland State University. Leslie B. Hammer, Department of Psychology, Portland State University.

This research was conducted as part of the Work, Family, and Health Network (www.WorkFamilyHealthNetwork.org), which is funded by a cooperative agreement through the National Institutes of Health and the Centers for Disease Control and Prevention: Eunice Kennedy Shriver National Institute of Child Health and Human Development (Grant \# U01HD051217, U01HD051218, U01HD051256, U01HD051276), National Institute on Aging (Grant \# U01AG027669), the National Heart, Lung and Blood Institute (R01HL107240), Office of Behavioral and Science Sciences Research, and National Institute for Occupational Safety and Health (Grant \# U01OH008788, U01HD059773). Grants from the William T. Grant Foundation, Alfred P Sloan Foundation, and the Administration for Children and Families have provided additional funding. The contents of this publication are solely the responsibility of the authors and do not necessarily represent the official views of these institutes and offices. Special acknowledgement goes to Extramural Staff Science Collaborator, Rosalind Berkowitz King, Ph.D. and Lynne Casper, Ph.D. for design of the original Workplace, Family, Health and Well-Being Network Initiative.

The first author was supported by the National Science Foundation under Grant No. DGE-1057604 during the writing of this manuscript. Any opinions, findings, and conclusions or recommendations expressed in this material are those of the authors and do not necessarily reflect the views of the National Science Foundation. 


\begin{abstract}
This study extends literature on workplace aggression and nonwork outcomes by examining the association between workplace aggression and employees' interactions with their children (i.e., parental warmth and child self-disclosure). Drawing on the Work-Home Resources model, the authors argue that experiencing workplace aggression drains employees' resources for effectively managing interactions with family members. Utilizing a longitudinal sample of long term healthcare workers with children aged 9-17 $(N=195)$, results of bias-corrected bootstrapped mediation tests provide support for a model in which perceived stress mediates the association between workplace aggression and parental warmth one year later. Theoretical and practical implications are discussed. Keywords: workplace aggression, perceived stress, parent-child relationships, spillover, work-family
\end{abstract}


The Role of Perceived Stress in the Relationship between Workplace Aggression and Parenting: Evidence from the Work, Family, \& Health Network Workplace aggression has been defined as actions or behaviors that cause harm, regardless of whether harm was intended (Edward, Ousey, Warelow, \& Lui, 2014). Workplace aggression can include both physical violence (e.g., pushing, bumping, grabbing, hitting, kicking, scratching, pulling hair, or biting) and psychological aggression (e.g., yelling, demeaning, swearing, threatening, false accusations of stealing or elder abuse; Åström et al., 2004). Estimates suggest that 40 - 90\% of U.S. employees experience workplace aggression on a yearly basis (Porath \& Pearson, 2010; Schat, Frone, \& Kelloway, 2006), and annual organizational costs associated with workplace aggression have been estimated at $\$ 100,000$ to $\$ 64$ billion (Farrell, 2002; Level Playing Field Institute, 2007; Leymann, 1990).

To date, most research on the implications of workplace aggression has focused on the work domain, including negative outcomes ranging from decreased job satisfaction and organizational citizenship behaviors, to increased turnover intentions, alcohol use, physical symptoms, burnout, and depression (Frone, 1999; Hershcovis \& Barling, 2010; Nixon, Mazzola, Bauer, Krueger, \& Spector, 2011). Recently, however, researchers have begun to identify negative impacts that extend beyond the workplace. For example, recent research has identified a positive association between workplace aggression and work family conflict (WFC; Carlson, Ferguson, Hunter, \& Whitten, 2012; Demsky, Ellis, \& Fritz, 2014; Lim \& Lee, 2011; Liu et al., 2014; Restubog, Scott, \& Zagenczyk, 2011). Such findings are consistent with the tenets of the Work-Home Resources model (ten Brummelhuis \& Bakker, 2012) which hold that work demands like 
workplace aggression can also influence functioning at home. Previous research has linked several work demands to parenting (Bass, Butler, Grzywacz, \& Linney, 2009; Bumpus, Crouter, \& McHale, 1999; Repetti \& Wood, 1997), however, we could find no study that examined the implications of workplace aggression for parenting. As such, this study responds to the call to investigate how the influence of workplace injustices (e.g., discrimination, harassment, abuse, and bullying) on employee health can spill over to influence employees’ family experiences (Okechukwu, Souza, Davis, \& de Castro, 2014).

Using a longitudinal sample of employed parents of children aged 9-17, the current study was designed to contribute to the literature on workplace aggression and nonwork outcomes in three ways. First, we build on recent literature that has identified nonwork outcomes of workplace aggression (Carlson et al., 2012; Demsky et al., 2014; Lim \& Lee, 2011; Liu et al., 2014; Restubog et al., 2011; Tepper, 2000), to examine the links between workplace aggression and characteristics of employees' parent-child relationships. In doing so, we also contribute to a growing body of research which links work stress and parenting behaviors (Bass et al., 2009; Bumpus et al., 1999). Second, we tested one possible mechanism through which this linkage may emerge - namely, employee-parents' perceived stress. Although much of the research on workplace aggression focuses on identifying antecedents and outcomes of workplace aggression (Hershcovis et al., 2007; Hershcovis \& Barling, 2010), relatively few studies have examined the processes through which workplace aggression is transmitted to work or nonwork outcomes. Finally, we examined our hypotheses with a longitudinal study design, in which workplace aggression and perceived stress were measured at baseline, 
while parenting outcomes were measured 12 months later. This design allows us to control for baseline levels of parenting behaviors as a way of examining the effects of workplace aggression and perceived stress above and beyond initial levels of parenting behaviors. In these ways we contribute to the literature by testing tenets of the WorkHome Resources Model (ten Brummelhuis \& Bakker, 2012), which hold that demands in either the work or home spheres deplete personal resources, with negative implications for the other domain (i.e., nonwork).

\section{Theoretical Background}

Work-Home Resources Model. Hobfoll (1989) proposed that stress occurs when an individual perceives a threat to existing resources, a loss of resources, or a failure to gain new resources after an investment of resources. Resources have been defined as objects, energies, conditions, or personal characteristics that an individual values, or that serve as a means of obtaining further resources (Hobfoll, 1989). Conservation of Resources (COR) theory is commonly drawn on to explain the link between threatened resources and individual levels of stress. In the current study, the experience of workplace aggression can be considered a threat to one's resources (i.e., emotional, cognitive), which in turn may be associated with higher levels of perceived stress. Building on COR theory, ten Brummelhuis and Bakker (2012) introduced the WorkHome Resources Model to explain the processes through which work can either enrich or conflict with the nonwork domain — or vise-versa. From this perspective work demands and resources have implications for individuals' personal resources, which are in turn associated with outcomes at home. This spillover process has been described as one in which either work-related or home-related stress results in stress in the other domain for 
the same individual, and is thus an interpersonal phenomenon (Bolger, DeLongis, Kessler, \& Wethington, 1989; Hanson, Hammer, \& Colton, 2006).

The first proposition of the Work-Home Resources Model states that "contextual work demands diminish home outcomes through a loss in personal resources (work-tohome conflict)" (ten Brummelhuis \& Bakker, 2012, p. 549). In the current study, we tested whether workplace aggression, a workplace demand, is associated with a depletion of personal resources (i.e., perceived stress), which is in turn associated with negative parent-child interactions at home. Workplace aggression can be conceptualized as an emotional demand, and given its highly interpersonal nature, can be seen as directly threatening one's social support network at work. In the current study, if the coping mechanisms used to address workplace aggression are unsuccessful or if many resources need to be invested to address this workplace demand, stress develops (Hobfoll, 2002). Experiencing aggression on the job may be seen as a threat to an employee's sense of connectedness, belonging, and respect.

\section{Workplace Aggression}

Workplace aggression has been defined as actions or behaviors that cause harm, regardless of whether harm was intended (Edward et al., 2014). Additionally, workplace violence is described as "any act or threat of physical violence, harassment, intimidation, or other threatening disruptive behavior that occurs at the worksite. It ranges from threats and verbal abuse to physical assaults and even homicide. It can affect and involve employees, clients, customers, and visitors" (OSHA, 2015). Although a number of related constructs have been examined in the organizational literature, including workplace violence, abusive supervision (Tepper, 2000), workplace bullying (Einarsen, 2000), 
social undermining (Duffy, Ganster, \& Pagon, 2002), and workplace incivility (Andersson \& Pearson, 1999), researchers have called for incorporating these similar constructs under the umbrella term of workplace aggression (Hershcovis, 2011) In the current study, we measure employee-parents' exposure to both psychological and physical forms of workplace aggression and refer to this range of experiences as workplace aggression. Occupations within the healthcare sector are a common focus of the workplace aggression literature, due to the high prevalence of aggression within this work context.

Workplace aggression in long-term care. Providing direct care to long-term care residents is associated with a high risk of experiencing workplace aggression (Gerberich et al., 2004). Healthcare industries account for $48 \%$ of all non-fatal injuries from assaults and violent acts. Staff at long-term care facilities have one of the highest annual rates of injury from assaults and violent acts, with 25 per 10,000 workers, compared to a rate of 2 per 10,000 among workers in the private sector overall (OHSA, 2004). Almost all (96\%) of long-term health care workers report experiencing some form of workplace aggression over their career (Zeller, Dassen, Kok, Needham \& Halfens, 2012). When asked about all forms of workplace aggression, 40-82\% of workers in the long term health care industry report experiencing workplace aggression in the last year (Åström et al., 2004; Morgan, Stewart, D’Arcy, Forbes \& Lawson, 2005; Zeller et al., 2012), and 38-77\% in the last week (Snyder, Chen \& Vacha-Haase, 2007; Zeller et al, 2012). Psychological aggression is also more common (76\%; Zeller et al., 2012) than physical violence (50-73\%; Miranda, Punnett, Gore \& Boyer, 2010; Miranda, Punnett, \& 
Gore, 2014). Reports of the prevalence of workplace violence vary as a function of methodological differences (Zeller et al., 2009).

Although the sources of workplace aggression toward direct care staff include patients, relatives and friends of patients, other direct care staff, and supervisors, the majority is perpetrated by residents (Taylor \& Rew, 2009). Studies indicate that patients are responsible for $65-99 \%$ of the workplace aggression reported by direct care staff (Gerberich, 2004; Taylor and Rew, 2009). When only physical violence is considered, patients are the source of 90-99\% of reports (Gerberich, 2004; Islam, Edla, Mujuru, Doyle, \& Ducatman, 2003). One contributing factor is the high prevalence of residents with dementia, physical limitations, and debilitating medical conditions which are associated with agitation, aggression, and combativeness (Geriatric Mental Health Foundation, 2014). For example, up to $86 \%$ of long-term care residents with dementia become aggressive during care (Beck, Rossby \& Baldwin, 1991; Beck et al., 1998; Ryden, Bossenmaier \& McLachlan, 1991). In addition, residents without cognitive disabilities may behave in aggressive ways toward workers (Gates, Fitzwater \& Meyer, 1999). Combined, these prevalence rates and contributing factors indicate that long-term healthcare workers are at high risk for experiencing workplace aggression, and as such, may be more likely to experience the negative outcomes associated with workplace aggression as well. In line with previous research, we propose that experiences of physical and psychological workplace aggression will be associated with increased perceived stress. A model of all hypothesized relationships can be seen in Figure 1.

H1: Workplace aggression is associated with increased perceived stress.

\section{Perceived Stress as a Mediator}


Perceived stress refers to an individual's subjective appraisal of the degree to which experiences in their life are appraised as being unpredictable, uncontrollable, and overloading (Cohen, Kamarck, \& Mermelstein, 1983). Measuring an individual's perceived stress has advantages over the use of objective life event lists, given that individuals' appraisals of those events are most closely related to health and well-being outcomes (Lazarus, 1966). In line with the Work-Home Resources Model (ten Brummelhuis \& Bakker, 2012), perceived stress can be conceptualized as a result of depleted personal resources; stress is proposed to occur when an individual's resources are threatened or lost. The experience of resource depletion as indicated by perceived stress will likely have negative implications for functioning at home. For example, parents may perceive higher levels of stress as a result of experiencing either physical or psychological aggression in the workplace over time. In turn, this perceived stress may manifest outside of the work domain at home, through reduced resources available for interacting and connecting with family members.

\section{Work Demands and Parenting}

In this study we focus on the implications of workplace aggression for workers' family experiences, specifically their interactions with their children. Previous research has demonstrated a link between various work demands and employees' parenting behaviors; however, we know little about the associations between workplace aggression and parent-child outcomes. For example, earlier research has examined associations between mothers' and fathers' work experiences, parenting, and child behaviors (MacEwen \& Barling, 1991; Stewart \& Barling, 1996). Fathers' work experiences (i.e., decision latitude, job demands, job insecurity, and interrole conflict) have been associated 
with children's behavior through the mechanisms of job-related affect and parenting behaviors (i.e., punishing \& rejecting behaviors; Stewart and Barling, 1996). Mothers' work experiences (i.e., interrole conflict and satisfaction) have also been associated with personal strain (i.e., cognitive difficulties, negative mood) and parenting behaviors (i.e., rejecting and punishing; MacEwen \& Barling, 1991).

The current study focuses on parental warmth and child disclosure as indicators of parent-child relationship quality. Parental warmth is typically viewed as an aspect of parenting characterized by supportiveness, affection, and sensitivity to the child's needs (Darling \& Steinberg, 1993). Child disclosure refers to a child spontaneously offering information about activities (Stattin \& Kerr, 2000). These two constructs were chosen as they provide insights into interactions between employee parents and children. Further, work-related stress has been associated with higher levels of both WFC and family-towork conflict (FWC; Allen, Herst, Bruck, \& Sutton, 2000; Amstad, Meier, Fasel, Elfering, \& Semmer, 2011; Byron, 2005). Of relevance to the current study's sample (i.e., extended care employees), meta-analytic evidence has also indicated that caregivers experience significantly greater levels of perceived stress than noncaregivers (Pinquart \& Sörenson, 2003).

Previous research has also linked parents' work experiences with similar parenting and relationship quality characteristics. Work demands such as long hours, restrictive organizational norms, nonstandard hours, work pressure, low supervisor support, and supervisor criticism have been associated with the disturbance of parentchild activities and reduced relationship quality (Gassman-Pines, 2011; Ransford, Crouter, \& McHale, 2008; Roeters, van der Lippe, \& Kluwer, 2010). Conversely, 
positive aspects of work (i.e., complexity, challenge, and stimulation) have been associated with developmentally sound parenting behaviors such as increased parental warmth and responsiveness (Greenberger, O’Neil, \& Nagel, 1994).

H2a-b: Perceived stress is associated with reduced levels of parental warmth (a) and child disclosure (b).

Accordingly, we hypothesize the following mediational process:

H3a-b: Workplace aggression will have an indirect effect on (a) parental warmth and (b) child disclosure via perceived stress. 


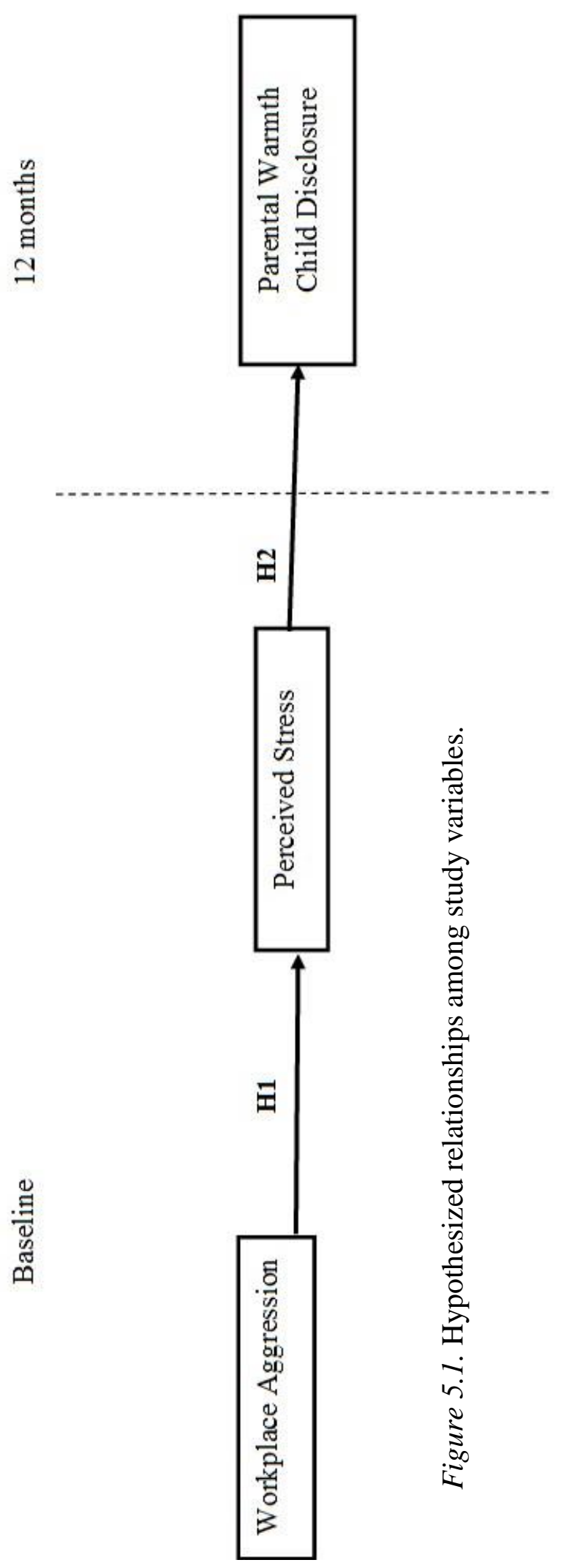




\section{Method}

\section{Participants and Procedure}

This study utilized baseline and 12 month follow-up data from the Work, Family, and Health Study (WFHS; Bray et al., 2013). The present study includes data from a sample of employees in the extended health care industry. Employees were eligible to participate in the study if they normally worked 22.5 or more hours per week in a direct patient care position, or a relevant position in the nursing department, and they worked on the day or evening shifts. Examples of job titles included licensed practical nurses (LPNs), registered nurses (RNs), and licensed or certified nurse assistants (CNAs).

Trained field interviewers administered face-to-face computer-assisted interviews (CAPI) with employees at their worksite and at home. Employee data collection at the worksite included a 60-minute interview and additional health assessments. Employees

received up to $\$ 60$ for participation in all work site collections at each wave of the study. For employees with a child aged 9-17, 25-minute home interviews were conducted at baseline and 12 months with a $\$ 30$ incentive at each wave. If participants had more than one age-eligible child, the child closet to age 13 was selected. Target children included biological, step-, and adopted children who lived with the employee four or more days per week. Of the 1783 eligible employees, 1524 employees completed the workplace CAPI (85.5\% response rate). For the home data collection, of 373 eligible employees, 257 completed the home CAPI (68.9\% response rate). After the 12-month follow-up, the final sample size for the current study was 195. 
On average, employees were 38.43 years of age $(S D=6.67)$ and worked 36.94 hours per week $(S D=8.41)$. The majority $(93.8 \%)$ of participants were female, while $6.2 \%$ were male. Most participants had finished some college or technical school $(52.1 \%)$, while $30.9 \%$ were high school graduates, $9.8 \%$ were college graduates, and 6.7\% finished some high school. Nearly half (48.5\%) were currently married, while an additional $17.5 \%$ currently lived with a romantic partner. The remaining $34 \%$ of the sample were currently single. The average target child age was $12.91(S D=2.14) ; 50.6 \%$ were female, while $49.4 \%$ were male. Participants had an average of 2.4 children $(S D=$ 1.14) living at home three or more days per week.

\section{Measures}

Workplace aggression. Workplace aggression was measured at baseline via two items (Dierdorff \&Ellington, 2008): (1) "How often is dealing with unpleasant, angry, or discourteous people a part of your current job? This includes both coworkers and residents" and (2) "How often is dealing with violent or physically aggressive people a part of your current job?" Items were rated on a scale from 1 (never) to 5 (every day) and averaged to create a mean score of workplace aggression. Given the two-item scale, we calculated the Spearman-Brown reliability coefficient which was .60 (Eisinga, te Grotenhuis, \& Pelzer, 2013).

Perceived stress. Employees' perceived stress was measured at baseline with the four-item Perceived Stress Scale (PSS; Cohen et al., 1983). An example item includes, "During the past 30 days, how often have you felt confident about your ability to handle your personal problems?" Items were scored on a scale of 1 (very often) to 5 (never). 
After reverse coding two items so that higher scores indicate a higher level of perceived stress, all scale items were summed. Cronbach's alpha for this scale was .73.

Parental warmth. Employees' warmth towards their children was measured at baseline and 12 months with an eight-item parent's version of the Child's Report of Behavior Inventory (CRPBI; Schaefer, 1965; Schwarz, Barton-Henry, \& Pruzinsky, 1985). An example item is, "You almost always speak to [Target Child] in a warm and friendly voice." Items were scored on a 1 (not at all) to 5 (very much) scale and were averaged so that higher scores indicate higher levels of parental warmth. Cronbach's alpha for this scale at baseline was .82 , and at 12 months was .88 .

Child disclosure. Parents reported on child disclosure at baseline and 12 months using four items developed by Stattin and Kerr (2000), including, "How often does [Target Child] tell you about how his/her day went without being asked?" Items were measured on a five-point scale, from 1 (almost never) to 5 (almost always) and were averaged so that higher scores indicate higher levels of disclosure. Cronbach's alpha at baseline was .80, and at 12 months was .83 .

Control variables. Several control variables were considered on the basis of previous research as well as theoretical rationale. Both age and gender $(1=$ male, $2=$ female) have been associated with workplace aggression (Barling, Dupre, \& Kelloway, 2009; Hershcovis et al., 2007). Age may be also be associated with length of experience as a parent, while gender has been consistently associated with parenting behavior (McKee et al., 2007; Wang, Repetti, \& Campos, 2011). Finally, data in the current study were collected as part of a larger intervention study, in which employees were assigned to either the intervention or control arm of an intervention aimed at reducing work-family 
conflict, thereby improving the health and well-being of employees, their families, and their workplaces (Kossek, Hammer, Kelly, \& Moen, 2014). Intervention status (1 = intervention, $2=$ control) was examined as a predictor and moderator of the proposed relations, though no significant effects emerged. Only gender emerged as a significant covariate during preliminary analyses, so all other proposed control variables (i.e., condition and employee age) were removed from subsequent models for the sake of parsimony (Aiken \& West, 1991).

\section{Results}

Table 1 shows descriptive statistics and inter-correlations among all study variables. On average, younger and single employees reported higher frequencies of exposure to workplace aggression. In line with the proposed hypotheses, workplace aggression was positively associated with perceived stress $(r=.20, p<.001)$ and 12 month reports of parental warmth $(r=-.15, p<.05)$. Perceived stress was correlated with all dependent variables in the expected directions, with the exception of baseline parental warmth. 


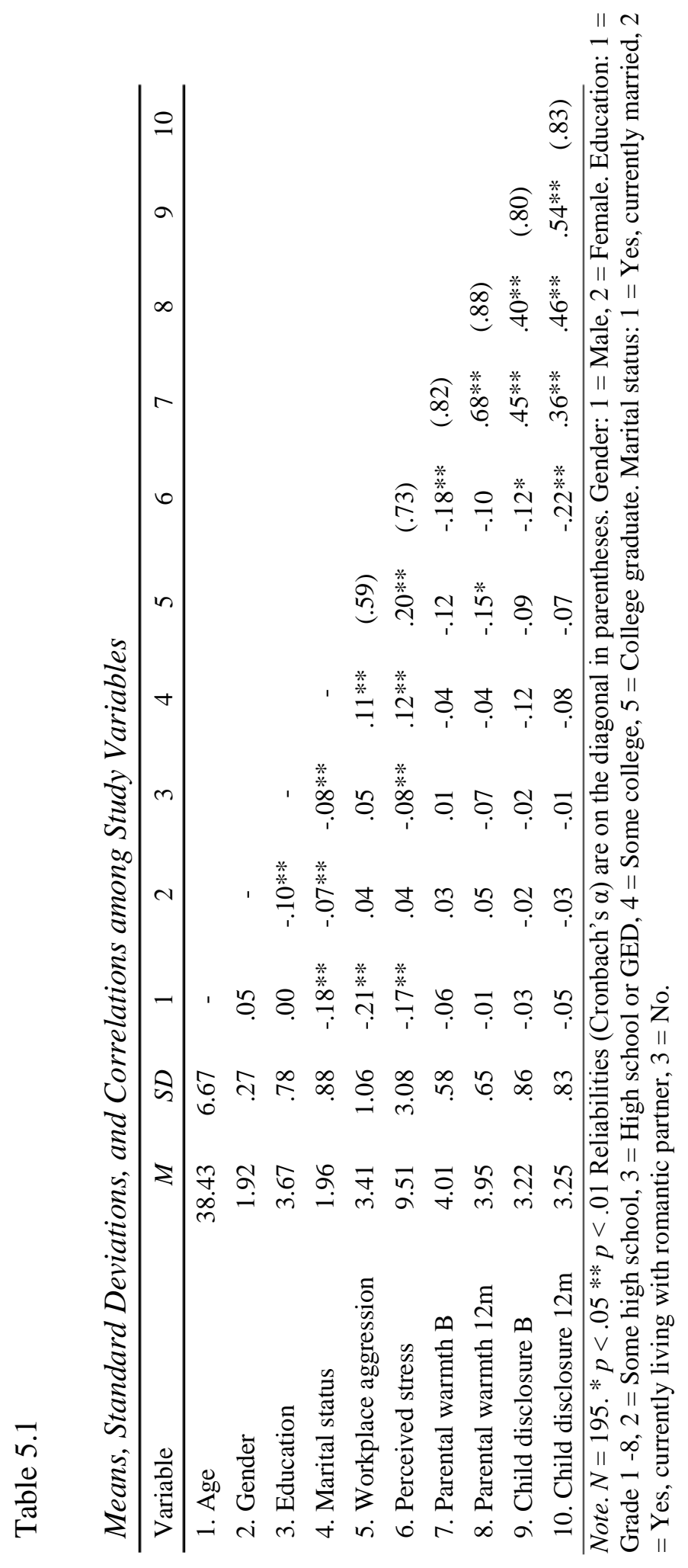


The hypothesized relationships were analyzed using MPlus 6.0 (Muthén \& Muthén, 1998 - 2010). Employee gender, baseline parental warmth, and baseline child disclosure were controlled for in all analyses. Our hypotheses were tested via the use of bias-corrected bootstrapped mediation tests as recommended by Preacher and Hayes (2004, 2008). Hypothesis 1 was supported, as the association between workplace aggression and perceived stress was significant $($ Est. $=.15, \mathrm{SE}=.13, p<.001,95 \% \mathrm{CI}$ : $.30, .82)$. Hypothesis 2 was partially supported, as perceived stress was significantly associated with parental warmth at 12 months (Est. $=-.30, \mathrm{SE}=.06, p<.001,95 \% \mathrm{CI}$ : $.48,-.23$; Hypothesis $2 \mathrm{a}$ ), but not with child disclosure at 12 months $($ Est. $=.02, \mathrm{SE}=.10$, $p=.67,95 \%$ CI: -.15, .24; Hypothesis $2 b)$. Finally, results indicate a significant indirect effect from baseline employee-reported workplace aggression on 12 month follow-up reports of parental warmth via baseline employee perceived stress $($ Est. $=-.20$, S.E. $=.06$, $p=.001,95 \%$ CI: $-.34,-.10)$. The indirect effect of baseline workplace aggression on 12 month reports of child disclosure was not significant (Est. $=.02$, S.E. $=.06, p=.67,95 \%$ CI: -.09, .15). These results indicate partial support for Hypothesis 3 (see Table 5.2). 


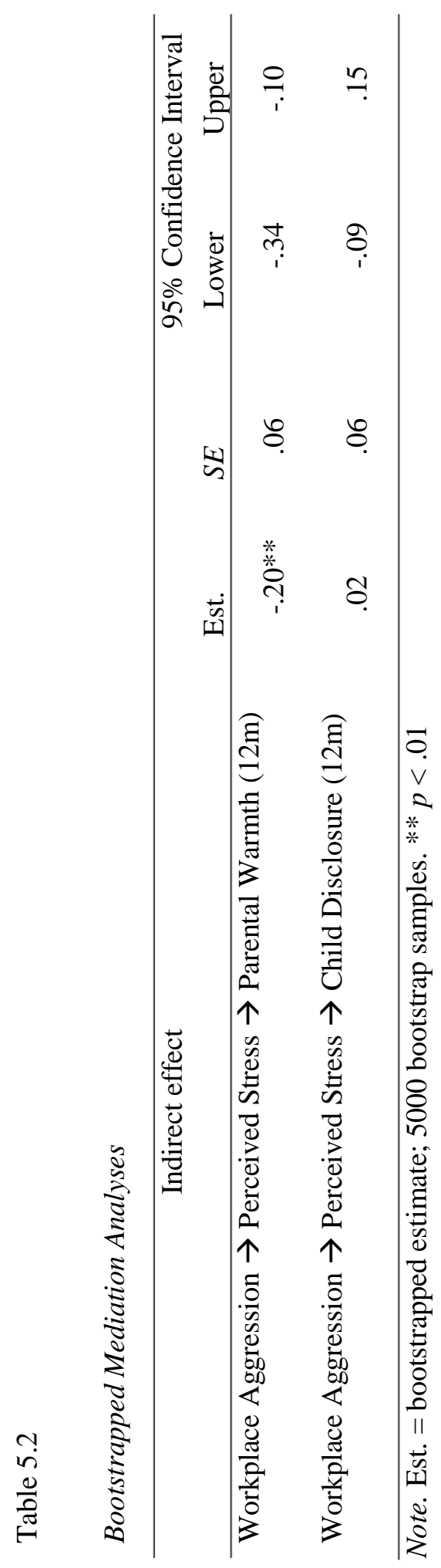




\section{Discussion}

Overall, the results of the present study suggest that workplace aggression is related to employee-parents' perceived stress, and in turn, diminished parental warmth. Evidence for longitudinal associations was found. These findings are in line with previous research on the associations between parents' work experiences and their parenting behaviors (e.g., Bass et al., 2009; MacEwen \& Barling, 1991; Stewart \& Barling, 1996). Based in the Work-Home Resources Model, these results provide important information on the negative impact that workplace aggression can have on family outcomes, and specifically on parenting outcomes. Research findings on the effects of workplace risk factors such as aggression on nonwork outcomes are still limited, and the present study adds to this growing body of literature.

The current study contributes to the literature on workplace aggression and parenting behaviors in several ways. Specifically, to date we know that workplace aggression has a number of negative effects for employees, ranging from negative job outcomes to poor health and well-being. Recently, several nonwork implications of workplace aggression have been identified as well, though parenting behaviors have previously not been included in these nonwork outcomes. Our results indicate significant downstream impacts of workplace aggression on parental warmth, even after controlling for baseline levels of parental warmth. These results suggest that the effects of workplace aggression may extend beyond the employee and their significant other.

Perceived stress was identified as one potential mechanism through which these downstream effects may occur; however, perceived stress did not mediate the relationship 
between workplace aggression and 12 month reports of child disclosure. There may be other mechanisms which explain this relationship - for example, parental mood may be a better predictor of a child's disclosure. Finally, our analyses allowed us to examine the effects of workplace aggression on parenting behaviors via perceived stress over a oneyear timespan, which adds to the literature on organizational stress (Zapf, Dormann, \& Frese, 1996) as well as recent theoretical developments (i.e., Work-Home Resources Model; ten Brummelhuis \& Bakker, 2012) calling for the explicit examination of resource depletion and generation between the work and nonwork domains.

\section{Practical Implications}

As previous research has shown, workplace aggression has wide-reaching implications for both employees and organizations. The present study contributes to our knowledge surrounding the association of workplace aggression with additional nonwork outcomes - specifically, how employee parents interact with their children during nonwork time. Organizations can make employees aware of the likelihood of spillover between the work and nonwork domains, and encourage employees to engage in activities that may diminish negative spillover. For example, recent research has identified a lack of psychological detachment as a mechanism through which workplace aggression is associated with work-to-family conflict (Demsky et al., 2014). Providing training on recovery experiences may be an opportunity to improve employees' wellbeing, both in the work and nonwork domains (Hahn, Binnewies, Sonnentag, \& Mojza, 2011).

It is important to note that in addition to providing employees with the resources to effectively manage workplace aggression, organizations should also actively seek to 
prevent workplace aggression from occurring in the first place. There are a number of steps management can take to prevent and address workplace aggression, including setting zero-tolerance policies, actively teaching civility, refusing to make excuses for powerful perpetrators, and taking the time to conduct exit interviews (Pearson \& Porath, 2005). Civility interventions have been shown to positively influence employee outcomes such as respect, job satisfaction, management trust, and absences, and can be a worthwhile endeavor for organizations seeking to reduce or prevent workplace aggression (Leiter, Laschinger, Day, \& Oore, 2011; Osatuke, Leiter, Belton, Dyrenforth, \& Ramsel, 2013). Additionally, we would propose that workplace interventions in the healthcare context move toward patient-centered care, which has been associated with less aggression (Hanson, Perrin, Moss, Laharnar, \& Glass, 2015). Offering training in deescalation techniques to residents as well as employees may also prove beneficial. Our current findings suggest the possibility that workplace interventions aimed at reducing workplace aggression may extend beyond the employee to also reach family members.

\section{Implications for Research}

The current study provides several important avenues for future research on workplace aggression and parenting behaviors. Our findings add to previous research on the long-term effects of workplace aggression (Carlson et al., 2012; Restubog et al., 2011; Trépanier, Fernet, \& Austin, 2015). To our knowledge, no studies have examined the association between workplace aggression and parent behaviors. In our current study, we find significant associations between workplace aggression and parental warmth twelve months later above and beyond the effects of baseline parental warmth, via increased perceived stress. This represents a major contribution to the burgeoning 
literature on workplace aggression and nonwork outcomes. It would be worthwhile to continue this line of inquiry, as workplace aggression may be associated with additional parenting behaviors or child adjustment outcomes.

While we found support for the indirect effect of workplace aggression on 12month follow-up reports of parental warmth via perceived stress, we did not find support for indirect effects on child disclosure. It is possible that these relations may unfold over a shorter timeline, and future research should consider examining these relations with different time lags, including at the day-level. As previous researchers have pointed out, time lags used in occupational health psychology often vary widely, and are often chosen due to organizational and design constraints rather than theory (Zapf et al., 1996). The Work-Home Resources Model proposes that short-term and long-term work-home conflict and enrichment processes will develop differentially over time, such that volatile demands and resources (i.e., fleeting, only able to be used once) will influence short-term processes, and structural demands and resources (i.e., stable, long-lasting) will influence long-term processes.

Additionally, although the current study sought to test a portion of the WorkHome Resources Model, the hypothesized relations only focused on the negative process of work-to-home conflict, and only in the direction of work-to-home (not home-to-work). Future tests of this theoretical model should be expanded to include an examination of the positive process of work-to-home enrichment (and conversely, home-to-work enrichment). The Work-Home Resources Model also posits that macro resources (i.e., characteristics of the larger environment in which a person is embedded) and key resources (i.e., management resources which facilitate the use of other resources, such as 
personality traits including optimism and intensity of goal purpose) may moderate the relationships between contextual demands and resources and personal resources. Future research should consider examining the moderating role of these resources (e.g., selfesteem, civility climate, etc.).

\section{Potential Limitations}

Although the current study offers several contributions to the literature, it is important to acknowledge its weaknesses as well. First, the mediator was assessed at the same time as the independent variable, which means we are unable to disentangle the directionality of this association (Selig \& Preacher, 2009). Although theoretically consistent, these relations should be explored using cross-lagged designs in future research studies. Second, the sample was long-term healthcare employees, an occupational group with its own set of work demands and resources including high levels of workplace aggression (Bureau of Labor Statistics, 2010; Gerberich et al., 2004), in addition to high levels of emotional and physical demands (Clausen, Nielsen, Carneiro, \& Borg, 2012; Van Der Heijden, Demerouti, Bakker, \& The NEXT Study Group, 2008). These factors may uniquely influence employees' stress and in turn, parenting. As such, future research should examine these processes in other occupational groups. Finally, although perceived stress may be one particular mechanism through which workplace aggression is transmitted to home outcomes, future studies should consider exploring additional mechanisms such as parent mood or parental time with children. Further, though we conceptualized perceived stress an indicator of resource depletion, it would be worthwhile to measure specific resources that may be depleted as a result of exposure to workplace aggression (e.g., positive affect, attentiveness, etc.). 


\section{Conclusion}

The current study identified perceived stress as a mechanism through which workplace aggression was associated with decreased parental warmth at 12 months, even after controlling for baseline levels of parental warmth. Our findings suggest that workplace aggression may have far-reaching implications for employees' interactions in the family domain. In addition to testing the Work-Home Resources model, our study contributes to the literature on workplace aggression by exploring the association between this workplace stressor and parenting. We also described several important theoretical and practical implications of this study, including suggestions for future research on the unfolding of these processes at the day-level. 
Chapter 6: Examining Understudied Work Outcomes of Workplace Aggression: Associations with Safety

In Study 1 and Study 2, workplace aggression was associated with negative outcomes in the nonwork domain - namely, increases in work-family conflict (WFC; Demsky, Ellis, \& Fritz, 2014) and decreases in parental warmth (Demsky \& Hammer, in progress). In addition to linking workplace aggression to these nonwork outcomes, the prior two studies examined two underlying mechanisms of this process (i.e., psychological detachment and perceived stress). Though this research contributes to the body of knowledge on outcomes of workplace aggression, much remains to be discovered regarding potential buffers of these relationships, such as personal and organizational resources. These resources may be particularly useful to researchers and practitioners alike when designing workplace interventions aimed at reducing the negative influence of workplace aggression. In line with Conservation of Resources theory (COR; Hobfoll, 1989) and the Job Demands-Resources model (Demerouti, Bakker, Nachreiner, \& Schaufeli, 2001), these resources may be able to protect against the negative effects of job demands such as workplace aggression. The third and final study of this dissertation seeks to examine the role of mindfulness, conceptualized as a personal resource, and safety climate, conceptualized as an organizational resource, as buffers of the relationship between workplace aggression and safety outcomes. Additionally, Study 3 continues the approach of Studies 1 and 2 by examining the underlying process through which workplace aggression is proposed to be associated with workplace safety (via cognitive failure and rumination). The mediation model tested in Study 3 can be seen in Figure 6.1. 


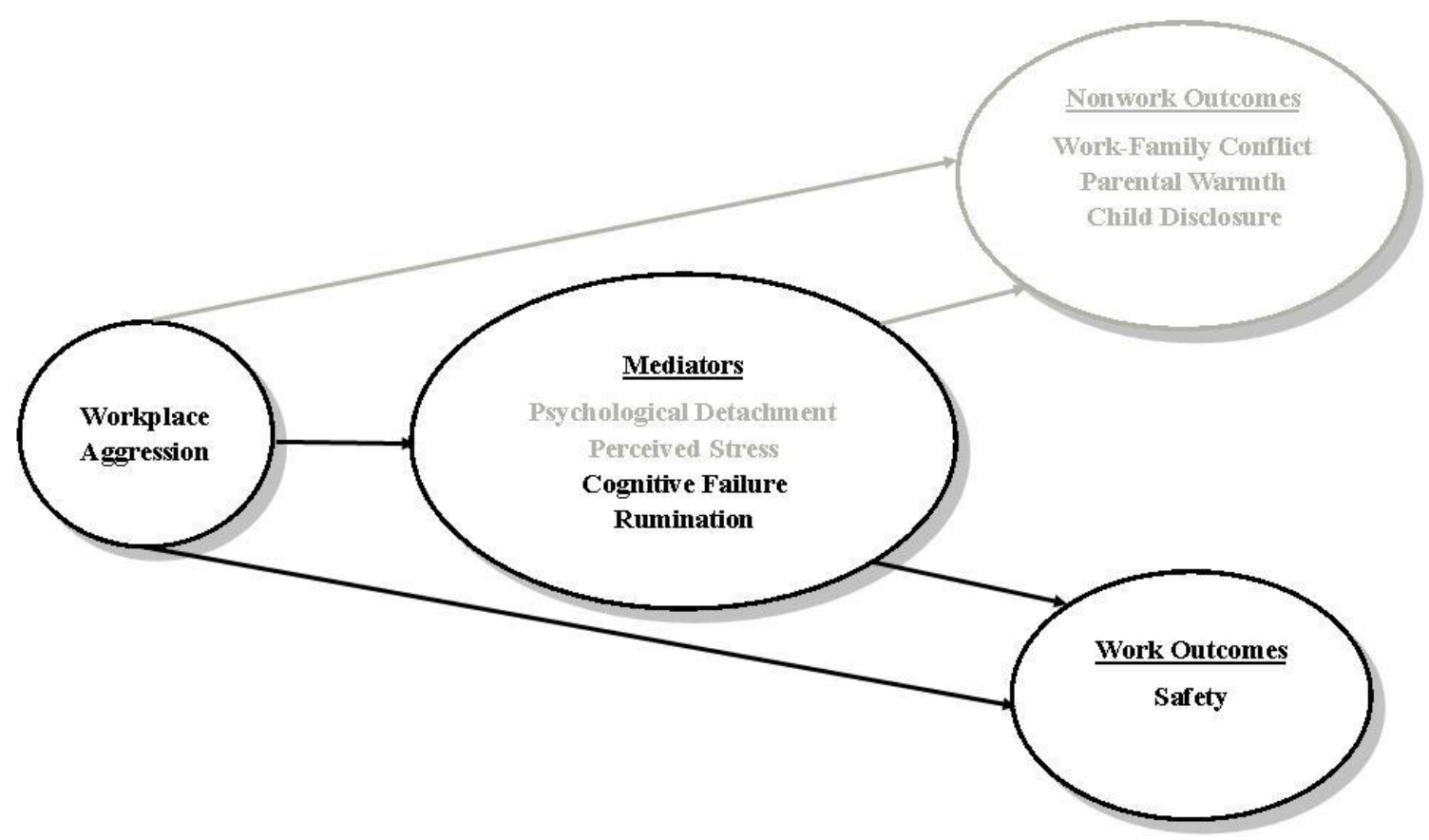

Figure 6.1. Summary model of the proposed relationships examined in Study 3. 
Chapter 7: Workplace Aggression and Occupational Safety: A Moderated Mediation

Model

Caitlin A. Demsky

Charlotte Fritz

Portland State University

(in preparation) 


\section{Author Note}

Caitlin A. Demsky, Department of Psychology, Portland State University; Charlotte Fritz, Department of Psychology, Portland State University.

This project was funded through a Research Joint Venture Agreement (\#14-JV11221611-109) between the United States Department of Agriculture Forest Service and Portland State University. All opinions expressed in this document are those of the authors' and do not necessarily represent the opinions of the USDA Forest Service. The first author was also supported during the course of this project by a National Science Foundation Graduate Research Fellowship under Grant No. DGE-1057604. Any opinions, findings, and conclusions or recommendations expressed in this material are those of the authors and do not necessarily reflect the views of the National Science Foundation.

Correspondence concerning this article should be addressed to Caitlin A. Demsky, Department of Psychology, Portland State University, P.O. Box 751, Portland, OR, 97207-0751, USA. E-mail: cademsky@pdx.edu 


\begin{abstract}
The current study examines a moderated mediation model in which the relationship between workplace aggression and employee safety is mediated by resource depletion (i.e., cognitive failure and rumination). Mindfulness, conceptualized as a personal resource, was hypothesized to moderate the first stage of the mediation, while safety climate, an organizational resource, was proposed to moderate the second stage of the mediation. A sample of 699 employees of the USDA Forest Service were utilized to examine the proposed moderated mediation. Overall, limited support was found for the current hypotheses. Both supervisor- and coworker-initiated workplace aggression were found to be associated with increased resource depletion, workplace accidents, and decreased safety compliance. Indicators of resource depletion were found to be differentially associated to safety, as cognitive failure was associated with safety behaviors, while rumination was associated with workplace injuries. Workplace aggression was indirectly associated with safety participation and workplace injuries via cognitive failure and rumination, respectively. Support was found for safety climate as a significant buffer of the workplace aggression and safety relationship, as it was found to moderate the relationship between rumination and safety behaviors. Additionally, the indirect effect coworker aggression on safety compliance via rumination and the indirect effect of supervisor aggression on safety participation via rumination was found to be conditional on low levels of safety climate. The findings of this study contribute to the literature on workplace aggression, as relatively little is known about the mechanisms through which workplace aggression is associated with employee safety. Additionally, support for the associations between mindfulness and safety climate and resource
\end{abstract}


depletion and employee safety provides promising avenues for future research and workplace interventions.

Keywords: workplace aggression, safety, resources, mindfulness, moderated mediation 
Workplace Aggression and Occupational Safety: A Moderated Mediation Model Recent prevalence estimates suggest that anywhere from $40-96 \%$ of U.S. employees have experienced psychological aggression in the workplace within the past year (Porath \& Pearson, 2010; Schat, Frone, \& Kelloway, 2006). Workplace aggression has been defined by researchers as efforts made by individuals to either harm others in the workplace, or the organizations by which they are currently or were previously employed (Neuman \& Baron, 1997). A wide range of similar constructs have been identified under the umbrella of workplace aggression, including abusive supervision (Tepper, 2000), mobbing (Duffy \& Sperry, 2007), workplace incivility (Lim \& Cortina, 2005), interpersonal conflict (Spector \& Jex, 1998), and workplace harassment (Bowling \& Beehr, 2006).

Research has indicated that workplace aggression has negative effects on a number of work and well-being outcomes - however, much less is known about the association between workplace aggression and safety outcomes. In the current study, we propose that workplace aggression is associated with decreased on-the-job safety through increased resource depletion, and further, that available personal and organizational resources may buffer these relationships. In addition to being a prevalent workplace phenomenon, meta-analytic evidence suggests that workplace aggression can have serious and detrimental outcomes for both employees and organizations. For example, workplace aggression is associated with decreased job satisfaction, affective commitment, physical well-being, and performance and increased turnover intentions, emotional exhaustion, depression, interpersonal deviance, and organizational deviance (Hershcovis \& Barling, 2010). For organizations, the effects of workplace aggression can 
be costly. Organizational costs associated with various forms of workplace aggression have been estimated as ranging from $\$ 100,000$ to $\$ 180$ million annually (Farrell, 2002; Leymann, 1990), depending on the severity of the aggression being examined.

While workplace aggression has been linked to a number of employee and organizational outcomes, few studies have examined the potential association between workplace aggression and safety outcomes, though other job stressors have been associated with decreased safety (Christian, Bradley, Wallace, \& Burke, 2009). This study will contribute to the workplace aggression literature by identifying an association between workplace aggression and safety outcomes (i.e., accidents, injuries, and safety behaviors). Safety is a particularly important outcome to examine, given its high impact on human and financial outcomes. In 2012, 4,628 fatal work injuries occurred in the United States, and 1,153,980 cases of occupational injuries and illnesses requiring days away from work were reported (Bureau of Labor Statistics, 2013, 2014). Worldwide, more than 2.3 million workplace fatalities are recorded each year. The economic costs of poor safety and health practices has been estimated at $4 \%$ of the yearly global Gross Domestic Product (International Labour Organization, n.d.). For these reasons, it is all the more important for researchers to understand and address the antecedents of unsafe work behaviors and practices.

Second, the current study will also examine two potential mechanisms by which this relationship occurs. Specifically, we hypothesize that cognitive failure and rumination will mediate the relationship between workplace aggression and safety outcomes. Finally, while a great deal of the research surrounding workplace aggression has focused on identifying antecedents and outcomes, fewer studies examine potential 
protective factors that may mitigate the impact of workplace aggression (see Schat \&

Kelloway, 2003 for one exception). The current study proposes two individual-level and organizational-level resources that may reduce the negative associations between workplace aggression and safety outcomes. Mindfulness is conceptualized as an individual-level resource, while safety climate is proposed as a relevant organizational resource. These resources are of particular importance as research has demonstrated the trainability of each (e.g., Kabat-Zinn, 1990; Zohar \& Polachek, 2014), making them particularly useful as targets for employers looking to reduce the negative effects of aggression in the workplace.

\section{Theoretical Background}

The theoretical framework for this study is drawn primarily from the Job Demands-Resources Model (Demerouti \& Bakker, 2011) and the Conservation of Resources theory (Hobfoll, 1989). In the hypothesized model (see Figure 7.1), a lack of resources is proposed to be one of the underlying mechanisms through which workplace aggression and safety outcomes are associated. Furthermore, we propose that the presence of personal and organizational resources (i.e., mindfulness, safety climate) will moderate the indirect effects of workplace aggression on safety outcomes. More specifically, we propose that workplace aggression indirectly affects safety outcomes through increased cognitive failure and rumination, while mindfulness (conceptualized here as a personal resource) moderates the first stage of this process, and safety climate (an organizational resource) moderates the second stage of this process. 
Job Demands-Resources Model. The Job Demands-Resources model (JD-R; Demerouti, Bakker, Nachreiner, \& Schaufeli, 2001) defines job demands as "physical, psychological, social, or organizational aspects of the job that require sustained physical and/or psychological (cognitive and emotional) effort or skills that are therefore associated with certain physiological and/or psychological costs" (Demerouti \& Bakker, 2011, p. 2). In occupations in which employees engage in a great deal of interpersonal communication, workplace aggression can become a particularly negative social aspect of the workplace, which may require sustained psychological effort to address. Therefore, in the current study, we conceptualize workplace aggression as a job demand. Job demands, whereas not inherently negative, can become stressful when meeting those demands requires sustained high effort. Job demands in general have been associated with increased burnout, which in turn can lead to poor health and organizational functioning (Schaufeli \& Bakker, 2004). More specifically, the literature surrounding workplace aggression has identified a number of potential costs for employees who find themselves on the receiving end of aggression, including decreased health and well-being and poor work outcomes (Hershcovis \& Barling, 2010).

In addition to examining the influence of workplace aggression on safety outcomes, the current study also examines two resources which may mitigate the influence of workplace aggression on safety outcomes. The JD-R model defines resources as physical, psychological, social, or organizational job aspects that can either serve a role in accomplishing work goals, minimize the impact of job demands, or generate growth, learning, and development (Demerouti \& Bakker, 2011). Research on the JD-R model has typically associated the presence of job resources with work 
engagement, which in turn can lead to increased well-being and positive organizational functioning (Bakker, Demerouti, \& Sanz-Vergel, 2014).

One of the propositions of the JD-R model is an interactive effect between job demands and resources. Job resources have been shown to attenuate the influence of job demands on strain outcomes (e.g., Bakker, Demerouti, \& Euwema, 2005; Xanthopoulou, Bakker, Demerouti, \& Schaufeli, 2007). Extensions of the JD-R model have included personal resources, which are typically linked to resiliency, and refer to an individual's sense that they are able to control and change their environment, particularly during stressful times (Hobfoll, Johnson, Ennis, \& Jackson, 2003). Personal resources (e.g., core-self evaluations, emotional stability) have been associated with a number of favorable work outcomes, including job performance and satisfaction (e.g., Judge, van Vianen, \& de Pater, 2004). In the current study, mindfulness is proposed as a personal resource that may offset the negative influences of workplace aggression. Additionally, safety climate is proposed as an organizational resource that may weaken the association between resource loss and safety outcomes. These hypotheses are in line with previous research proposing interactive effects between job demands and resources.

Conservation of Resources Theory. Conservation of Resources theory (COR) posits that individuals experience stress when resources are threatened, lost, or not gained after an investment of resources (Hobfoll, 1989). COR theory defines resources as objects, energies, personal characteristics, or conditions that are valued, or that which are used to obtain further resources. COR theory also posits that resource loss is more salient than resource gain, and that resources must be invested in order to gain additional resources, prevent the loss of resources, or recover from resource loss (Hobfoll, 1998, 
2001). In the context of the current study, workplace aggression is seen as a threat to one's resources. Exposure to this workplace stressor may deplete employees' resources (e.g., cognitive, emotional), in turn leading to detrimental workplace outcomes.

In the current study, we propose that resource loss will manifest as cognitive failure and rumination. As employees experience resource loss, they have fewer resources to invest in work-related responsibilities, which may include attending to safety-related roles and responsibilities. With fewer resources to invest in safety-related behaviors, it is expected that employees will experience higher levels of work-related accidents and injuries, and engage in fewer safety behaviors. It is hypothesized that both personal resources (i.e., mindfulness) and organizational resources (i.e., safety climate) that are available for investment may mitigate the associations between workplace aggression, resource loss, and safety-related outcomes. Both mindfulness and safety climate can be conceptualized as resources in accordance with COR theory - specifically, as personal and contextual resources. Hobfoll (2002) described personal resources as those that are proximal to the self, such as mood, attention, or resilience. Mindfulness may be described as a more stable personal resource, as it is has been identified in the literature as a dispositional state that is susceptible to intervention (Brown \& Ryan, 2003). Contextual resources are described as being outside the self and within the social contexts an individual is located within (Hobfoll, 2002). Safety climate, in this instance, would be a stable characteristic of one's work environment that could be drawn upon repeatedly to promote safe work behaviors. 


\section{Workplace Aggression and Resource Depletion}

Workplace aggression, a workplace stressor consisting of negative acts by employees aimed at either directly or indirectly harming coworkers or the organization (Neuman \& Baron, 1997), has been identified as having a number of detrimental outcomes for those who experience it. Several researchers have attempted to explain the processes through which workplace aggression leads to poor health, well-being, and work outcomes, with several potential mechanisms having been proposed in the literature. One such explanation describes the process through which exposure to certain triggering events (e.g., abusive supervision, rigid policies) may lead to cognition-initiated processing, and in turn to further aggressive acts (Douglas et al., 2008). The current study focuses on two potential mechanisms of resource loss: cognitive failure and rumination. In line with the JD-R model, workplace aggression is seen as a job demand that is stressful and depleting for employees to the degree to which the demand exceeds their resources. As workplace aggression depletes an employee's resources, we expect that this will manifest itself as higher levels of cognitive failure and rumination.

Cognitive failure has been defined as a "cognitively based error that occurs during the performance of a task that the person is normally successful in executing" (Martin, 1983, p. 97). Generally, cognitive failure is conceptualized as execution lapses in the areas of attention, memory, and action (Wallace \& Chen, 2005). The current study will focus on the attention aspect of cognitive failure, which refers to failures in perception. Research has demonstrated that occupational stress is positively associated with cognitive failure. For example, one study found that burnout symptoms were significantly positively related to the number of cognitive failures in daily life (van der Linden, 
Keijsers, Eling, \& van Schaijk, 2005). Task stressors (i.e., time pressure, concentration demands, uncertainty, interruptions, and performance constraints) have also been positively associated with the attention subdimension of workplace cognitive failure (Elfering, Grebner, \& Dudan, 2011). We propose that high levels of workplace aggression will be positively associated with cognitive failures.

Work-related rumination refers to perseverative thinking about work-related problems, concerns, or issues during nonwork time (Cropley, Michalianou, Pravettoni, \& Milward, 2012). Although some employees may find themselves thinking positively about work-related successes or attempting to solve work-related problems during nonwork time, others may find themselves unable to cease thinking about stressful or negative aspects of the work environment. As researchers have identified unwinding from work as a critical component in preventing work demands from causing health problems, it is worth exploring characteristics of the work environment that may hinder or facilitate this unwinding process (Zijlstra \& Sonnentag, 2006). Experimental research has shown that rumination mediates the relationship between interpersonal provocation, provocation-induced negative affect, and displaced aggression, such that those who were instructed to ruminate on a provocation experienced higher levels of provocation-induced negative affect and engaged in higher levels of displaced aggression (Bushman, Bonacci, Pedersen, Vasquez, \& Miller, 2005). In another recent study, supervisors' history of family aggression was associated with higher levels of angry rumination, which was in turn associated with higher levels of abusive supervision in the workplace (Garcia, Restubog, Kiewitz, Scott, \& Tang, 2014). Workplace bullying, a form of workplace aggression, is also positively correlated with anger rumination (Moreno-Jimenez, 
Rodriguez-Munoz, Pastor, Sanz-Vergel, \& Garrosa, 2009). As such, we hypothesize that workplace aggression will be positively associated with rumination.

Hypothesis $1 a-b$ : Workplace aggression will be positively associated with resource depletion (i.e., cognitive failure, rumination).

\section{Workplace Aggression and Safety Outcomes}

To date, very little research has examined the potential link between experienced workplace aggression and workplace safety outcomes. In general, occupational stressors have been associated with increased accidents and injuries, typically through the employee's response to the stressors. These responses to occupational stressors may include a preoccupation with the event, distraction, or an emotional response, which interferes with an employee's ability to safely conduct their job (Mandler, 1979). The decreased attentional resources available during these responses may lead to increased errors, which can be particularly problematic in occupations that are already at high risk for accidents and injuries on the job (Wadsworth, Moss, Simpson, \& Smith, 2003).

Theoretically, workplace aggression can be conceptualized as a hindrance stressor (e.g., situational constraints, hassles, role conflict and interpersonal conflict, organizational politics), which are viewed as being difficult or unlikely for an employee to overcome (Podsakoff, LePine, \& LePine, 2007). Generally, research has found that hindrance stressors are negatively associated with performance (Wallace, Edwards, Arnold, Frazier, \& Finch, 2009). Meta-analytic evidence has demonstrated that hindrance stressors are associated with reduced safety behaviors (i.e., safety compliance, safety participation), as well as higher levels of occupational injuries and near-misses. Safety behaviors were found to fully mediate the relationship between hindrance stressors and 
occupational injuries (Clarke, 2012). Additionally, in line with COR theory, addressing the source of workplace aggression may deplete employees of resources (e.g., attentional) needed to address safety concerns in the environment, particularly in a dynamic work environment.

Research has generally linked occupational stressors to increased workplace accidents and injuries (Abbe, Harvey, Ikuma, \& Aghazadeh, 2011; Ahlberg-Hulten, Theorell, \& Sigala, 1995; Bigos et al., 1991; Goldenhar, Williams, \& Swanson, 2003; Hemingway \& Smith, 1999; Spector, Coulter, Stockwell, \& Matz, 2007; Yang, 2009), however fewer studies have focused on the specific link between workplace aggression and safety outcomes, and those that have report mixed findings. For example, Bruk Lee (2006) failed to find a significant relationship between interpersonal conflict and accidents in a sample of participants from diverse occupations. Other researchers have identified significant relationships between physical violence and psychological aggression and injuries among nurses (Spector et al., 2007), as well as accidents resulting in contagious disease exposure and musculoskeletal injuries (Nixon, 2011; Yang, 2009). Based on the existing research, it is likely that workplace aggression may be more strongly related to safety outcomes in occupations with a high risk of accidents and injuries.

Hypothesis $2 a$-d: Workplace aggression will be negatively associated with employee safety.

\section{Resource Depletion and Safety Outcomes}

Engaging in safety behaviors at work requires attention, forethought, and the capacity to distinguish between safe and unsafe workplace behaviors. Employees who are 
lacking the necessary cognitive, emotional, or physical resources to recognize potential safety concerns may be at greater risk for experiencing accidents and injuries on the job. Drawing on COR theory, a deficit of resources may in turn leave employees without resources to invest in work-related safety behaviors (e.g., following safety rules, wearing personal protective equipment). The JD-R model can also be used to explain this process, such that job demands may deplete an employee's resources, which in turn increases burnout. Job resources, on the other hand, are associated with higher levels of work engagement. Employees lacking resources may be more likely to injure themselves or experience an accident on the job, while those employees high in resources and work engagement may be more likely to actively engage in safety behaviors on the job. Metaanalytic evidence has found some support for this mediational process (Nahrgang, Morgeson, \& Hofman, 2011). Of relevance to the current study, burnout was found to partially mediate the relationship between job demands and adverse events. Additionally, safety compliance and burnout partially mediated the relationship between safety climate and adverse events.

Though limited research has been conducted on the association between cognitive failure and safety outcomes, existing findings note a significant association between the two. For example, Wallace and Vodanovich (2003a) found that individuals' proneness to experiencing cognitive failures was positively associated with self-reported work-related accidents. Other existing research has found positive associations between proneness to cognitive failure and automobile accidents (Larson \& Merrit, 1991; Larson, Alderton, Neideffler, \& Underhill, 1997). Cognitive failures have also been significantly positively associated with workplace accidents and unsafe work behaviors (Wallace \& Vodanovich, 
2003b). In their development of a measure of workplace cognitive failure, Wallace and Chen (2005) found positive associations between workplace cognitive failure and days away from work, work-related injuries, and restricted work days, and negative associations with safety compliance. More recent research found that cognitive failure scores mediated the relationship between individuals' level of general health and workplace accident occurrence (Day, Brasher, \& Bridger, 2012). These findings suggest that individuals who are stressed are more likely to experience an accident in the workplace due to cognitive failures. While much less research has focused on the mediating role of rumination as a predictor of safety outcomes, research has shown that ruminating about one's work during nonwork time can impede the process of recovery (Demerouti, Bakker, Geurts, \& Taris, 2009), which may result in fewer resources being available for investment at work the following day. Research has demonstrated that ruminative thinking exacerbates the effects of workplace violence on employee health and well-being, suggesting that rumination may play a role in the association between workplace aggression and employee outcomes (Niven, Sprigg, Armitage, \& Satchwell, 2013).

Hypothesis 3a-h: Resource depletion (i.e., cognitive failure, rumination) will be negatively associated with employee safety.

In summary, we propose: 1) that workplace aggression will be associated with resource depletion; 2) that workplace aggression will be associated with workplace safety; and 3) that resource depletion will be associated with workplace safety. Further, we propose that the relationship between workplace aggression and safety will be partially mediated by resource depletion, which we conceptualize in the current study as 
cognitive failure and rumination. We hypothesize a partial mediation and not a full mediation as it is possible that other variables not measured in the current study may also mediate the relationship between workplace aggression and workplace safety. For example, Yang (2009) found that emotional strain partially mediated the relationship between workplace aggression and workplace injuries in a sample of nurses.

Additionally, as previous research has indicated that workplace aggression may spill over to the nonwork domain via different mechanisms (e.g., negative affect, lack of psychological detachment, perceived stress; Volmer, Binnewies, Sonnentag, \& Niessen, 2012; Demsky, Ellis, \& Fritz, 2014; Demsky \& Hammer, in progress), it is likely that multiple mechanisms are also at play when examining the relationship between workplace aggression and work-related outcomes.

Hypothesis $4 a-h$ : The relationship between workplace aggression and employee safety will be partially mediated by resource depletion (i.e., cognitive failure, rumination).

\section{The Role of Mindfulness}

The concept of mindfulness has roots in Buddhist and related contemplative traditions, and has been defined as "the state of being attentive to and aware of what is taking place in the present" (Brown \& Ryan, 2003, p. 822). Brown and Ryan found that individuals naturally vary in their levels of mindfulness, and are predisposed to either mindful or mindless states. Individuals scoring higher on dispositional measures of mindfulness also experienced higher states of mindfulness in their everyday lives. In the current study, mindfulness is proposed to moderate the relationship between workplace aggression and resource loss (i.e., cognitive failure, self-regulation failure). Drawing on 
both COR and JD-R theory, mindfulness is conceptualized here as a personal resource (Bakker et al., 2014; Hobfoll, 2002), which may buffer against the negative outcomes associated with workplace aggression. In line with COR theory, mindfulness can be conceptualized as a personal resource that can be drawn upon to protect against resource loss.

Evidence for a moderating effect of mindfulness is particularly useful for workplace interventions, as research has suggested that mindfulness-based stress reduction therapy (MBSR) and mindfulness cognitive therapy are effective in reducing problematic outcomes such as depression and suicidal behaviors among predisposed individuals (Kabat-Zinn, 1990; Kingston, Dooley, Bates, Lawlor, \& Malone, 2007; Linehan et al., 2006; Segal, Williams, \& Teasdale, 2002). MBSR interventions have also been shown to increase levels of trait mindfulness, however, MBSR participants who have higher levels of mindfulness prior to the intervention see larger gains from such interventions (Shapiro, Brown, Thoreson, \& Plante, 2011).

Mindfulness practices and mindfulness based therapy have been associated with a number of positive effects, including effects on both mental and physical health (Grossman, Niemann, Schmidt, \& Walach, 2004). One meta-analysis by Eberth and Sedlmeier (2012) compared the effects of meditation practices and mindfulness based stress reduction programs (MBSR). In general, both practices were associated with positive effects, including decreased stress, anxiety, and negative emotions, and increased mindfulness, well-being, attention, positive emotions, self-realization, and emotion regulation. MBSR programs demonstrated stronger effects on well-being and negative emotions, though meditation showed stronger effects on mindfulness. 
Mindfulness has been associated with a number of positive outcomes, including improved psychological well-being (e.g., anxiety, depression, and positive affect), higher levels of autonomy, competence, and relatedness, and lower levels of physical symptoms and frequency of medical visits (Brown \& Ryan, 2003; Feldman, Hayes, Kumar, Greeson, \& Laurenceau, 2007). Mindfulness has also been indirectly associated with decreased emotional exhaustion and increased job satisfaction through the mechanism of surface acting (Hulsheger, Alberts, Feinholdt, \& Lang, 2013). In addition to directly influencing outcomes, mindfulness has also been shown to moderate the relationship between neuroticism and trait anger, as well as neuroticism and depressive symptoms (Feltman, Robinson, \& Ode, 2009). Trait mindfulness also moderates the association between MBSR techniques and outcomes such as mindfulness, subjective well-being, empathy, hope, and perceived stress (Shapiro et al., 2011). Mindfulness has also been shown to be negatively associated with rumination, a mediating variable in the current study (Brown \& Ryan, 2003; Feldman et al., 2007; Raes \& Williams, 2010). Similarly, trait mindfulness has been associated with fewer cognitive failures (Herndon, 2008).

Typically, workplace aggression is a stressor that elicits frustration and anger from those who experience it (Keenan \& Newton, 1985). Reacting to such workplace stressors is often associated with negative thought patterns, characteristic of rumination.

Individuals high in mindfulness, however, will be aware of their thoughts and reactions, but can avoid evaluating them as positive or negative (Glomb, Duffy, Bono, \& Yang, 2011). Accordingly, workplace aggression will pose less of a threat to one's resources, as it is not perceived as requiring high levels of effort to manage. Conversely, individuals with low levels of mindfulness may have a more difficult time acknowledging and 
detaching from negative thought patterns associated with experiencing workplace aggression. As such, it is hypothesized that dispositional mindfulness will weaken the association between workplace aggression and indicators of resource depletion.

Hypothesis 5a-b: The relationship between workplace aggression and resource depletion (i.e., cognitive failure, rumination) is moderated by mindfulness such that employees with higher levels of mindfulness will report lower levels of resource depletion, even under situations of high workplace aggression.

\section{The Role of Safety Climate}

Safety climate has been defined as a coherent set of shared organizational perceptions and expectations regarding safety (Zohar, 1980). Researchers have examined safety climate as a multi-dimensional construct, and measures of safety climate typically include some combination of the following dimensions: a) perceived management attitudes toward safety, b) perceived effects of safe behavior on promotion opportunities, c) perceived effects of safety behavior on social status within the organization, d) perceived organizational status of the safety officer, e) perceived importance and effectiveness of safety training, f) perceived risk level at the workplace, and g) perceived effectiveness of enforcement as compared to guidance in promoting safety (Zohar, 1980, p. 98). While safety climate is described as a coherent set of shared perceptions, there is also often variability in these perceptions within the same organization. Prior research has demonstrated that these variations may occur in part due to supervisor discretion in the implementation of formal procedures related to safety and productivity (Zohar \& Luria, 2005). 
COR theory would suggest that safety climate can be viewed as a structural contextual resource, as it is an ongoing part of one's environment that can be drawn upon repeatedly to protect against resource loss, or to invest in further resources (Hobfoll, 2002). JD-R would suggest that safety climate can be seen also as a job resource, as it is an organizational aspect of the work environment that can both serve a purpose in accomplishing work goals as well as generating growth, learning, and development (Demerouti \& Bakker, 2011).

A great deal of research has examined the role of safety climate in predicting and explaining occupational safety in the workplace over the last thirty years. Meta-analytic evidence has suggested that safety climate is associated with decreased burnout, accidents, injuries, and increased safety compliance, safety participation, safety knowledge, and safety motivation (Christian, et al., 2009; Clarke, 2006; Nahrgang et al., 2011). Additionally, researchers have examined safety climate as a moderator of several important relationships. For example, safety climate has been shown to moderate the effects of high-quality leader-member exchange (LMX) relationships on safety citizenship role definitions, such that role definitions were expanded under positive safety climates (Hofmann, Morgeson, \& Gerras, 2003). Work-unit level safety climate has also been shown to moderate the relationship between perceived colleagues' safety knowledge and safety behavior on employee safety behaviors. Under conditions of high unit-level safety climate, the relationship between perceived colleagues' safety knowledge and safety behavior and employees' safety behavior was stronger (Jiang, Yu, Li, \& Li, 2010). Importantly, recent research has identified that safety climate is capable of being changed via targeted organizational interventions (Zohar \& Polacheck, 2014). We propose that 
safety climate will moderate the relationship between resource depletion and safety

outcomes, as it can be drawn upon to protect against further resource loss (e.g., involvement in an accident).

Hypothesis 6a-h: The relationship between resource depletion (i.e., cognitive failure, rumination) and employee safety is moderated by safety climate, such that employees reporting high levels of safety climate will experience higher levels of safety, even under high levels of resource depletion.

\section{The Moderated Mediation Model}

While there is a very large literature on moderation and mediation analyses (e.g., Aiken \& West, 1991; Baron \& Kenny, 1986; Kenny, Kashy, \& Bolger, 1998), more recently, methodologists have begun to identify ways in which these analyses can be combined in theoretically valid and interesting ways. Moderated mediation occurs when "the mediating process that is responsible for producing the effect of the treatment [independent variable] on the outcome depends on the value of a moderator variable" (Muller, Judd, \& Yzerbyt, 2005, p. 854).

In the current study, it is hypothesized that the effect of workplace aggression on the proposed mediators (i.e., cognitive failure, rumination) will be moderated by mindfulness (Hypotheses 7a-b), while the effect of the mediators on the outcome variables are proposed to be moderated by safety climate (Hypotheses 8a-b). Hypotheses 7a-b propose that the mediated effect of workplace aggression will differ for individuals depending on their level of mindfulness. Specifically, those low in mindfulness should experience a stronger relationship between workplace aggression and resource depletion. 
Hypotheses 8a-b suggest that the mediated effect of resource depletion will be weaker for those experiencing high levels of safety climate.

Hypothesis 7a-h: Mindfulness will moderate the indirect effects of the proposed mediation. The effects of the mediation will be weaker for individuals high in mindfulness than for individuals low in mindfulness.

Hypothesis $8 a-h$ : Safety climate will moderate the indirect effects of the proposed mediation. The effects of the mediation will be weaker for those individuals reporting high levels of safety climate than for those individuals reporting low levels of safety climate. 

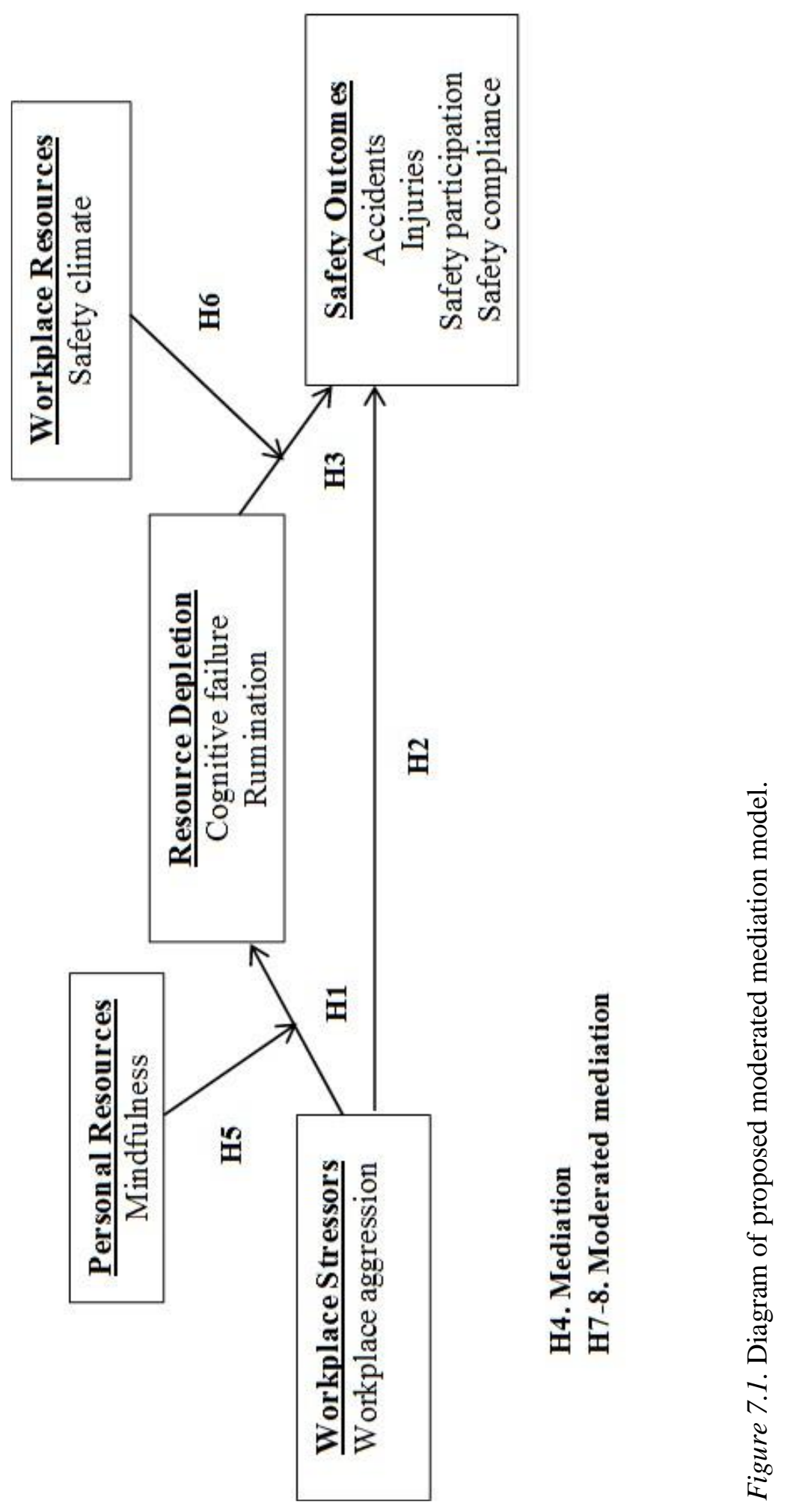


\section{Method}

\section{Participants and Procedure}

Employees of the United States Department of Agriculture (USDA) Forest

Service were recruited to participate in the current study. The present investigation was part of a larger survey-based examination of the occupational safety, health, and wellbeing of Forest Service employees. All full-time employees in the Southwest Region were contacted via email by a Deputy Regional Forester describing the project and expressing support for employee participation. The following week, participants received an email from the research team with additional information about the study, including a link to the online survey. Participants were asked to complete a one-time online survey, which took approximately 15-20 minutes to complete.

Of a potential 2,256 employees, 781 accessed the survey (34.6\% response rate). Of the 781 participants who accessed the survey, 699 provided useable data $(31 \%$ response rate). On average, participants were 48 years old $(S D=10.84)$, with 16.67 years $(S D=10.12)$ of experience with the USDA Forest Service and 6.95 years $(S D=6.23)$ of experience in their current position. $49.2 \%$ of the sample was female, while $47.2 \%$ was male. An additional $3.6 \%$ of the sample chose not to disclose their gender. The majority of participants (71.6\%) self-identified as White (non-Hispanic), while 16.5\% identified as Hispanic/Latino, 6.2\% identified as "Other," 3.8\% identified as Native American, 0.9\% identified as African American, 0.7\% identified as Asian, and 0.4\% identified as Native Alaskan or Pacific Islander. Regarding education, 45.9\% of participants had earned a 4year college degree, $28.5 \%$ had earned an advanced degree, $16.2 \%$ had attended some college, $5.8 \%$ had a 2 -year college degree, and 3.6\% had a high school diploma or GED. 
A total of $41 \%$ of participants classified their current jobs as supervisory, while $59 \%$ were in non-supervisory positions. On average, participants reported working five days a week $(S D=.42)$ and 41.77 hours per week $(S D=10.30)$. Participants reported working nearly four hours of overtime per week $(M=4.44 ; S D=13.37)$. Based on openended responses to this question, the amount of overtime employees worked varied largely during the fire season. $66.2 \%$ of participants were married, while $11.2 \%$ were single. A further $9.7 \%$ were divorced, $6.3 \%$ were currently in a relationship (not married), $5.4 \%$ were living with a partner, and $1.3 \%$ were widowed. Regarding children, $30.7 \%$ of the sample reported having one or more children under the age of 18 living at home at least half time.

\section{Measures}

Workplace aggression. Workplace aggression was measured using the Workplace Incivility Scale (Cortina, Magley, Williams, \& Langhout, 2001). Workplace incivility has been described as "low-intensity deviant behavior with ambiguous intent to harm the target, in violation of workplace norms for mutual respect" (Andersson \& Pearson, 1999, p. 457). Seven items refer to coworker-initiated incivility, and another seven items refer to supervisor-initiated incivility. Response options are on a 1 (never) to 5 (most of the time) point scale. Participants were asked how often coworkers or supervisors had engaged in uncivil behavior over the past six months (e.g., "put you down or were condescending to you," "made demeaning or derogatory remarks about you," and "ignored or excluded you from professional camaraderie"). This scale has previously been established as reliable ( $\alpha=.89$; Cortina et al., 2001). Cronbach's alpha was .93 for both supervisor incivility and coworker incivility in the current study. 
Cognitive failure. Three items developed by Wallace and Chen (2005) were used to assess the attentional dimension of cognitive failure. Respondents were asked to indicate how frequently they experienced each item in the past six months $(1=$ never to 5 =always). Example items include, "How often did you not focus your attention on work activities at work?" and "How often were you easily distracted by coworkers at work?" Wallace and Chen (2005) found acceptable reliability for this scale $(\alpha=.85)$; however Cronbach's alpha for this scale in the present study was .67. While the original scale developed by Wallace and Chen (2005) consisted of five items, the five-item scale demonstrated a poor level of reliability in the current sample $(\alpha=.62)$. In order to improve the reliability of the scale, two poorly performing items were removed from the scale. Further details on these items can be found in the Appendix.

Rumination. Affective rumination was measured with five items (Cropley et al., 2012). An example item is, "Are you troubled by work-related issues when not at work?" Response options ranged from 1 (very seldom or never) to 5 (very often or always). Prior research has found high levels of reliability for this scale $(\alpha=.90$; Querstret \& Cropley, 2012), while Cronbach's alpha in the current study was .96.

Mindfulness. Mindfulness was measured using seven items from the Five Facet Mindfulness Questionnaire which refer to nonreactivity to inner experiences (Baer, Smith, Hopkins, Krietemeyer, \& Toney, 2006). Example items include, “I perceive my feelings and emotions without having to react to them" and "In difficult situations, I can pause without immediately reacting." Response options ranged from 1 (never or very rarely) to 6 (very often or always true). Cronbach's alpha for the scale in the current study was .87 , which is higher than previously identified ( $\alpha=.75$; Baer et al., 2006). 
Safety climate. Safety climate was measured with three items developed by Neal, Griffin, and Hart (2000). Participants were asked to respond on a five-point scale $(1=$ strongly disagree to $5=$ strongly agree) the degree to which they agree with whether or not management emphasized safety. A sample item is "Management places a strong emphasis on workplace health and safety." Prior research indicates a high level of reliability for this scale ( $\alpha=.94-.95$; Neal \& Griffin, 2006). Cronbach's alpha for the scale in the current study was .93 .

Safety. Workplace accidents were assessed using a previously developed question (Hayes, Perander, Smecko, \& Trask, 1998), which asked participants to report how many accidents they had experienced in the last twelve months that were a) reported accidents, b) unreported accidents, and c) near accidents. Responses to this question were open ended. A composite accident variable was created by summing these three items. Workplace injuries were assessed with two questions (Barling, Kelloway, \& Iverson, 2003). These questions were: "Have you experienced an injury on the job in the last year?" and "If yes, how many days were you off of work as a result of your injury?" The first question was recorded as presence or absence of injury, while the second question indicates injury severity, with longer time off of work indicating increased injury severity. Safety compliance was measured using three items (Neal et al., 2000). An example item is, "I use the correct safety procedures for carrying out my job" $(\alpha=.90)$. Safety participation was also measured using three items (Neal et al., 2000), an example of which is, "I put in extra effort to improve the safety of the workplace" $(\alpha=.88)$. Response options ranged from 1 (strongly disagree) to 5 (strongly agree). Prior research indicates a high level of reliability for both safety compliance $(\alpha=.92-.93)$ and safety 
participation ( $\alpha=.86-.89$; Neal \& Griffin, 2006). Safety compliance and safety

participation are comparable to task performance and contextual performance,

respectively, as described by Borman and Motowidlo (1993). Safety compliance refers to

following established safety-related policies and procedures in line with one's job

description, while safety participation, like contextual performance, contributes to

creating a positive organizational environment for safety and often includes behaviors

that are outside of one's official job description.

Control variables. Several control variables were selected for inclusion based on theoretical rationale, empirical evidence, and relationships with both the independent and dependent variables. Specifically, in all analyses, we controlled for gender, supervisor status, and hours worked per week. Previous research has acknowledged that men tend to engage in and experience more workplace aggression than women (Schat et al., 2006). Additionally, individuals in supervisory roles may be less susceptible to experiencing workplace aggression from coworkers or subordinates, and may additionally have access to resources that buffer effects of workplace aggression (e.g., disciplinary procedures, flexible work assignments and schedules). Additionally, increased hours of work per week may be associated with more opportunity for exposure to workplace aggression, particularly in the current occupational context, when long hours frequently co-occur within stressful or dangerous working environments (e.g., wildland firefighting).

\section{Analytic Strategy}

As participants are employed within naturally-occurring work groups (e.g., National Forests and Ranger Districts), intraclass correlations (ICCs) were examined to determine the level of dependency within groups. For all predictor variables, ICCs ranged 
from .00 to .08 , indicating a lack of significant between group variance to warrant a multi-level analytic approach. However, larger ICCs at the Ranger District level for the outcome variable of workplace accidents (.19) suggested the need for multilevel modeling. In an effort to account for nesting within the data, multilevel models were specified with Ranger District as the nesting variable. We experienced convergence issues in a majority of the multilevel models. The moderated mediation model was tested in Mplus 6.0 (Muthén \& Muthén, 1998 - 2010). For the sake of parsimony, we report the results of the single-level moderated mediation models.

\section{Results}

Means, standard deviations, and inter-correlations are reported in Table 7.1. At the correlational level, study variables were largely associated in the expected directions. Supervisor aggression was positively associated with coworker aggression $(r=.41, p<$ $.001)$, affective rumination $(r=.41, p<.001)$, cognitive failure $(r=.13, p=.001)$, workrelated accidents $(r=.09, p=.03)$, and workplace injuries $(r=.09, p=.024)$, and negatively associated with safety climate $(r=-.36, p<.001)$, mindfulness $(r=-.15, p<$ $.001)$, and safety compliance $(r=-.09, p=.03)$. Similarly, coworker aggression was positively associated with affective rumination $(r=.28, p<.001)$, cognitive failure $(r=$ $.17, p<.001)$, work-related accidents $(r=.10, p=.016)$ and negatively associated with safety climate $(r=-.30, p<.001)$, mindfulness $(r=-.16, p<.001)$, and safety compliance $(r=-.13, p=.002)$. Neither supervisor aggression $(r=-.04, p=.30)$ nor coworker aggression $(r=.03, p=.54)$ was significantly associated with safety participation. 
Though mindfulness and safety climate were not hypothesized to be directly associated with any of the outcome variables, several associations were identified while examining preliminary results. Mindfulness was negatively associated with resource depletion (cognitive failure, $r=-.20, p<.001$; affective rumination, $r=-.24, p<.001$ ) and positively associated with employee safety: safety compliance $(r=.20, p<.001)$, and safety participation $(r=.22, p<.001)$. Safety climate also demonstrated significant associations with lower resource depletion (affective rumination, $r=-.20, p<.001$ ) and higher levels of safety: safety compliance $(r=.41, p<.001)$; safety participation $(r=$ $.30, p<.001)$; and workplace accidents $(r=-.09, p=.03)$. Mindfulness and safety climate were also significantly and positively associated with one another $(r=.16, p<$ $.001)$. 


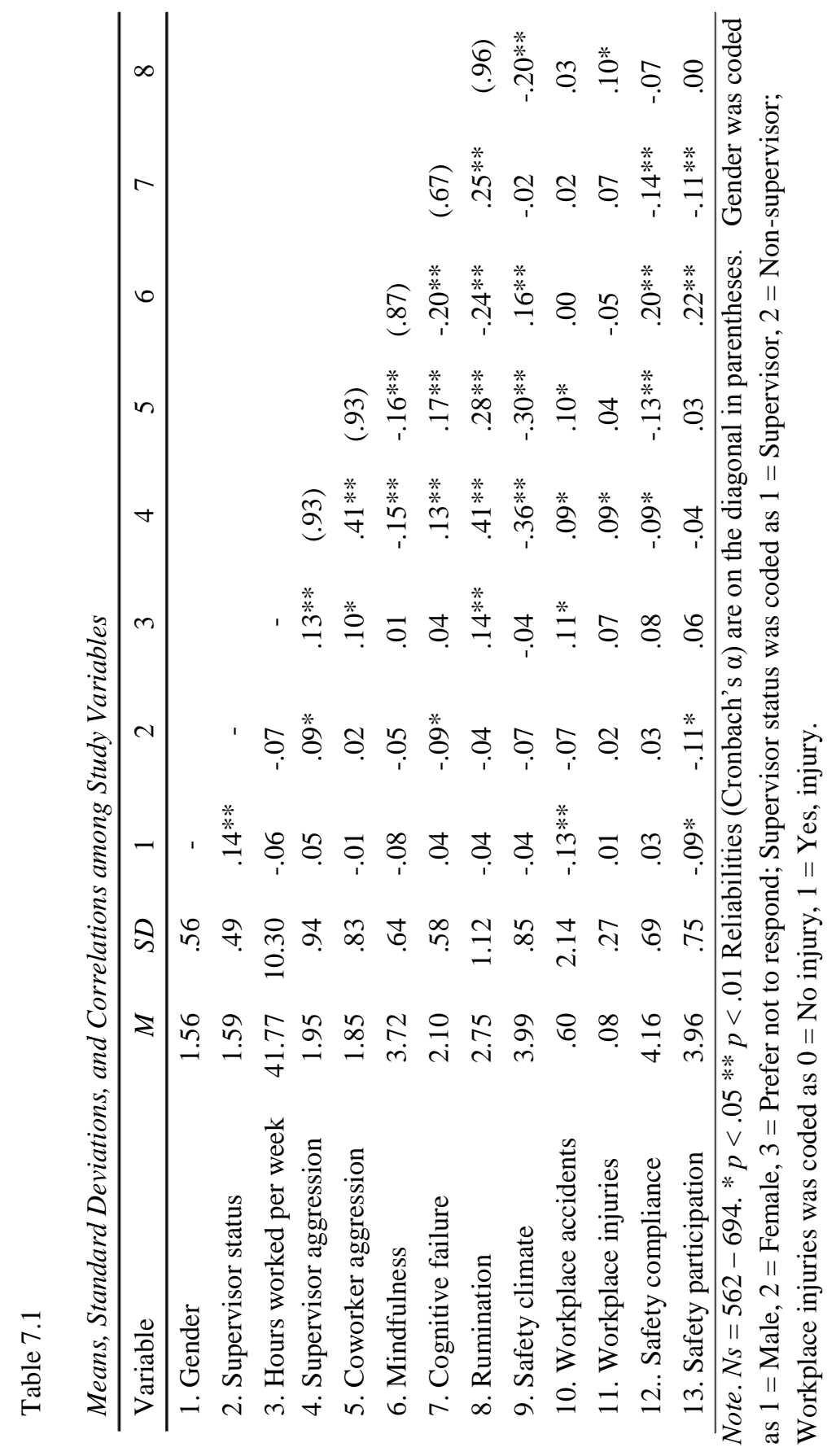




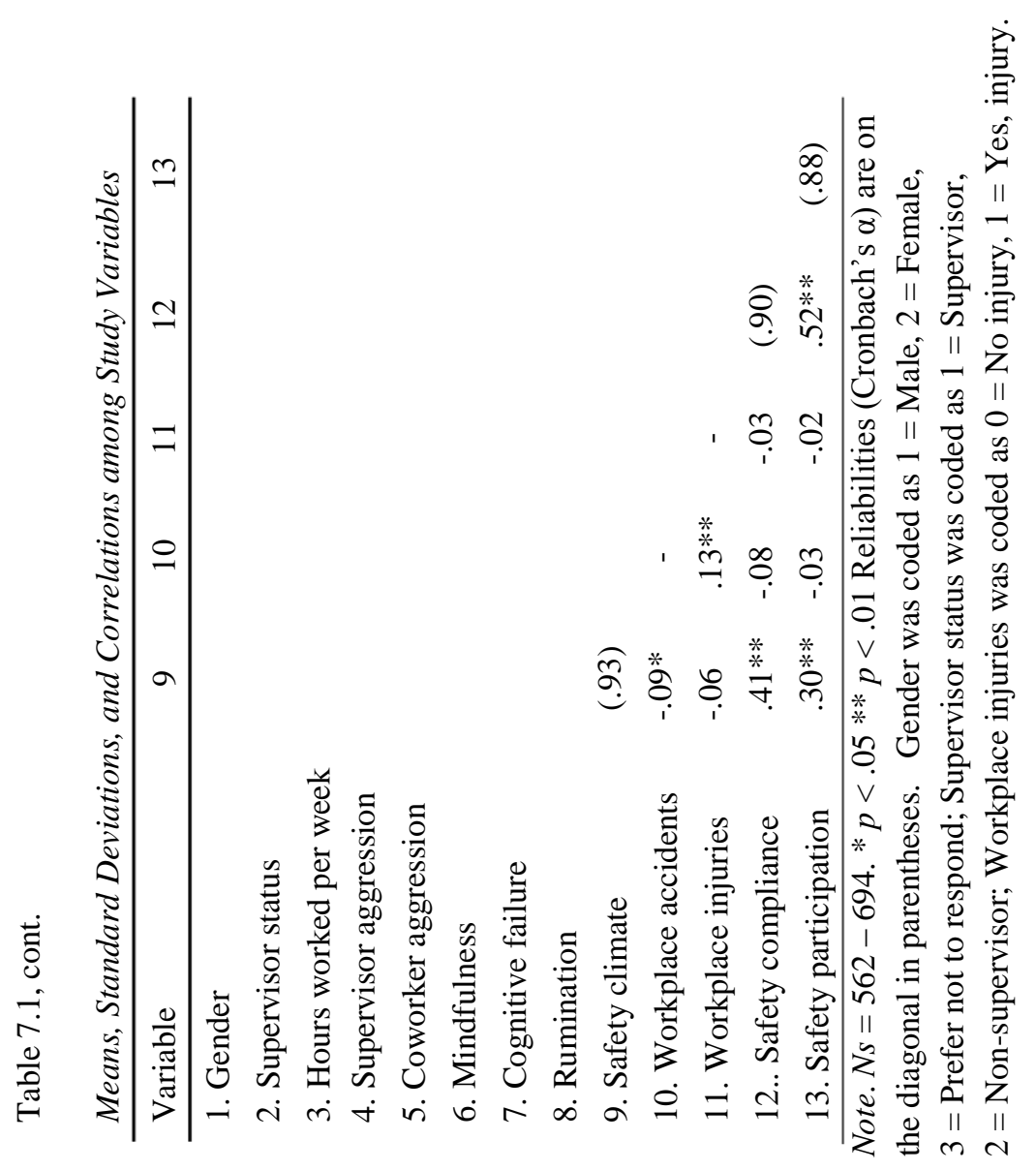




\section{Hypothesis Testing}

Main effects. Hypothesis 1 was tested using hierarchical multiple regression in

SPSS 17.0. Hypotheses $1 \mathrm{a}-\mathrm{b}$ proposed that workplace aggression would be positively associated with cognitive failure and rumination. After controlling for gender, supervisor status, and hours worked per week, supervisor aggression was significantly associated with cognitive failure $(\beta=.15, t=3.42, p=.001)$ and affective work-related rumination $(\beta=.40, t=10.14, p<.001)$. Coworker aggression displayed similar relationships with both cognitive failure $(\beta=.17, t=3.94, p<.001)$ and affective work-related rumination $(\beta=.27, t=6.57, p<.001)$, providing support for Hypothesis 1.

Hypotheses $2 \mathrm{a}-\mathrm{d}$ proposed that workplace aggression would be associated with increased accidents and injuries, and decreased safety compliance and safety participation. Poisson regression was used to test hypothesis $2 \mathrm{a}$, as workplace accidents represent a count variable of relatively infrequent events. Both supervisor aggression $(\beta=$ .32 , Wald Chi-square $=5.34, p=.021)$ and coworker aggression $(\beta=.32$, Wald Chisquare $=5.48, p=.019)$ were positively associated with work-related accidents, providing support for Hypothesis 2a. Logistic regression was used to test hypothesis $2 \mathrm{~b}$, as workplace injuries was a dichotomous variable $(0=$ No Injury; $1=$ Yes, Was Injured $)$. Controlling for gender, supervisor status, and hours worked per week, supervisor aggression was marginally associated with the log odds of experiencing a workplace injury, $\beta=.29, \mathrm{SE}=.15$, Wald $\chi^{2}$ statistic $=3.67, p=.056^{5}$. Coworker aggression was not

\footnotetext{
${ }^{5}$ When the control variables of gender, supervisor status, and hours worked per week were removed from the model, supervisor aggression was significantly associated with the log odds of experiencing a workplace injury, $\beta=.33, \mathrm{SE}=.15$, Wald $\chi^{2}$ statistic $=4.94, \mathrm{p}=.026$.
} 
significantly associated with the log odds of experiencing a workplace accident, $\beta=.17$, $\mathrm{SE}=.18$, Wald $\chi^{2}$ statistic $=.96, p=.33$, failing to provide support for Hypothesis $2 \mathrm{~b}$. Hierarchical regression analyses provided support for Hypothesis 2c, as both supervisor $\operatorname{aggression}(\beta=-.11, t=-2.44, p=.015)$ and coworker aggression $(\beta=-.13, t=-3.14, p$ $=.002$ ) were negatively associated with safety compliance. Neither supervisor aggression $(\beta=-.04, t=-.92, p=.36)$ nor coworker aggression $(\beta=.04, t=.92, p=.36)$ were associated with safety participation, failing to provide support for Hypothesis $2 \mathrm{~d}$.

Hypotheses 3a-b were not supported, as cognitive failure was not significantly associated with exposure to workplace accidents $(\beta=.15$, Wald Chi-square $=.39, p=$ $.53)$ or workplace injuries $\left(\beta=.41, \mathrm{SE}=.25\right.$, Wald $\chi^{2}$ statistic $\left.=2.71, p=.10\right)$.

Hypotheses 3c-d were supported, as cognitive failure was negatively associated with both safety compliance $(\beta=-.17, t=-3.24, p=.001)$ and safety participation $(\beta=-.16, t=-$ $2.87, p=.004)$. Regarding rumination, Hypothesis 3e was not supported, as rumination was not associated with workplace accidents $(\beta=.01$, Wald Chi-square $=.001, p=.98)$. Hypotheses $3 \mathrm{f}$ was supported, as rumination was significantly associated with the log odds of experiencing a workplace injury $\left(\beta=.30\right.$, Wald $\chi^{2}$ statistic $\left.=4.71, p=.03\right)$. Hypotheses $3 \mathrm{~g}$-h were not supported, as rumination was not significantly associated with safety compliance $(\beta=-.07, t=-1.71, p=.09)$ or safety participation $(\beta=.003, t=.07$, $p=.94)$.

Hypothesis 4: Mediation. Hypothesis 4 was addressed via the use of biascorrected bootstrapped mediation models, as recommended by Preacher and Hayes (2004, 2008). Tests of indirect effects were conducted via the use of MEDIATE and INDIRECT macros (Hayes \& Preacher, 2014; Preacher \& Hayes, 2008), which allow for 
the testing of multiple independent variables and mediators in one model, as well as the examination of dichotomous dependent variables. 5,000 bootstrapped resamples were requested from the obtained data, as well as $95 \%$ bias-corrected confidence intervals (CIs). The indirect effect is considered statistically significant when the upper and lower boundaries of the corrected CIs do not contain zero.

Results of these bias-corrected bootstrapped mediation models can be seen in Table 7.2. Overall, limited support was found for a significant indirect effect of workplace aggression on workplace safety via rumination or cognitive failure $($ Hypothesis 4a-h). Both supervisor aggression $($ Est. $=-.0105$, S.E. $=.0073,95 \%$ CI: $.0307,-.0003)$ and coworker aggression $($ Est. $=-.0161$, S.E. $=.0039,95 \%$ CI: $-.0159,-$ .0010) were found to be significantly indirectly associated with safety participation via cognitive failure, providing support for Hypothesis 4d. Further, coworker aggression was found to be significantly indirectly associated with the log odds of experiencing a workplace injury via increased rumination $($ Est. $=.1069$, S.E. $=.0580,95 \%$ CI: .0066 , .2370), providing partial support for Hypothesis $4 \mathrm{f}$.

A significant indirect effect was identified from both supervisor aggression and coworker aggression to safety compliance via cognitive failure (H4c). However, the test of homogeneity of regression was statistically significant, $F(2,529)=5.10, p=.006$, indicating the presence of a potential interactions between supervisor and coworker aggression and the mediator, cognitive failure. In this case, indirect effects of the independent variable on the dependent variable should not be interpreted (Hayes $\&$ Preacher, 2014). Though not initially proposed, these interactions were probed to determine potential significant relationships. Cognitive failure did not significantly 
moderate the relationship between supervisor aggression and safety compliance $(\beta=.07$, $t=-1.66, p=.10)$. However, cognitive failure did significantly moderate the relationship between coworker aggression and safety compliance $(\beta=-.10, t=-2.17, p=.03)$, such that safety compliance was lowest under conditions of high cognitive failure and high coworker aggression. A simple slopes test indicated that the slopes were significantly different from zero at both one standard deviation above $(t=-2.60, p=.01)$ and below $(t$ $=-2.92, p=.004)$ the mean of the moderator (cognitive failure). This interaction can be seen in Figure 7.2.

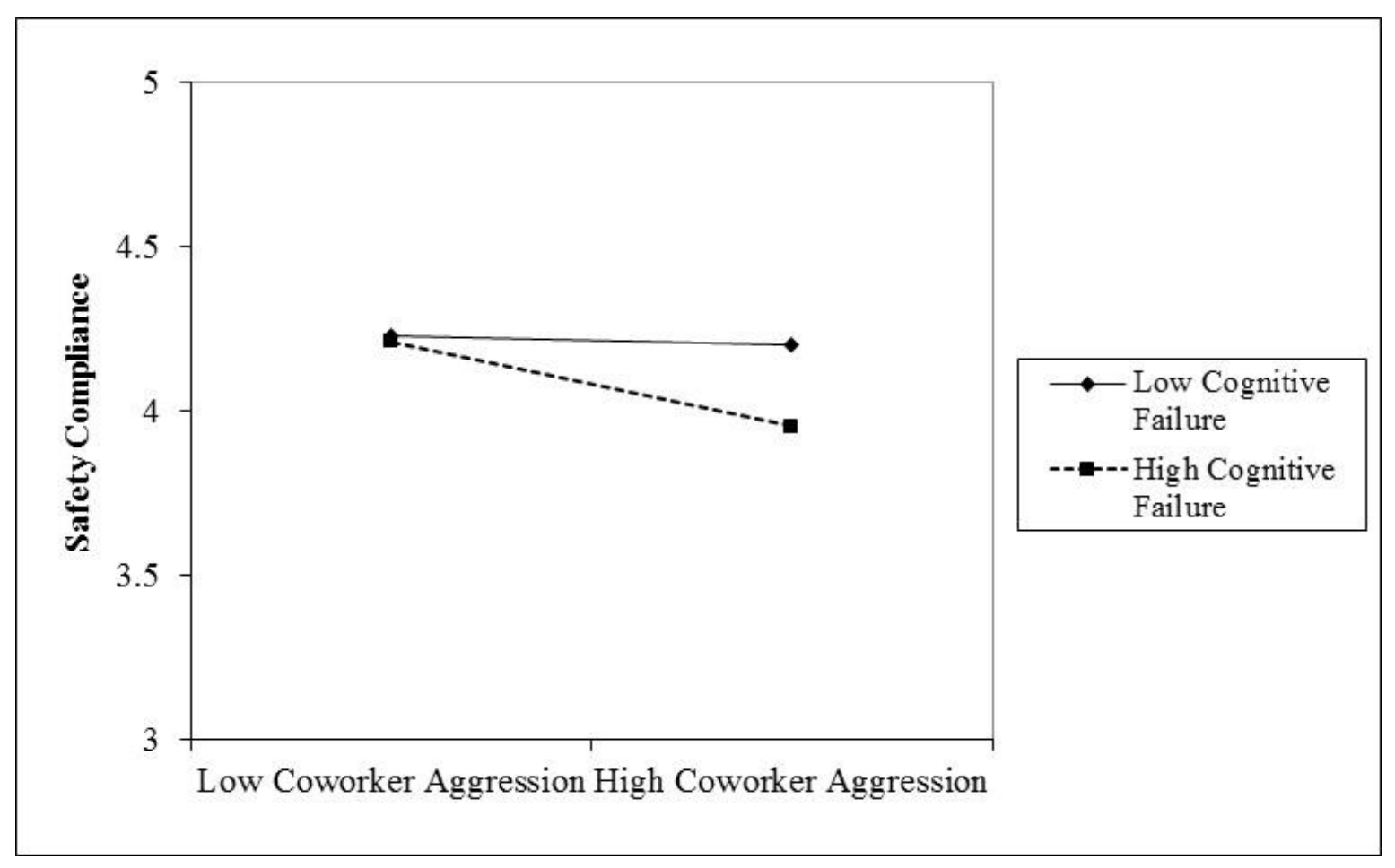

Figure 7.2. Exploratory analysis: Relationship between coworker aggression and safety compliance, moderated by cognitive failure. 


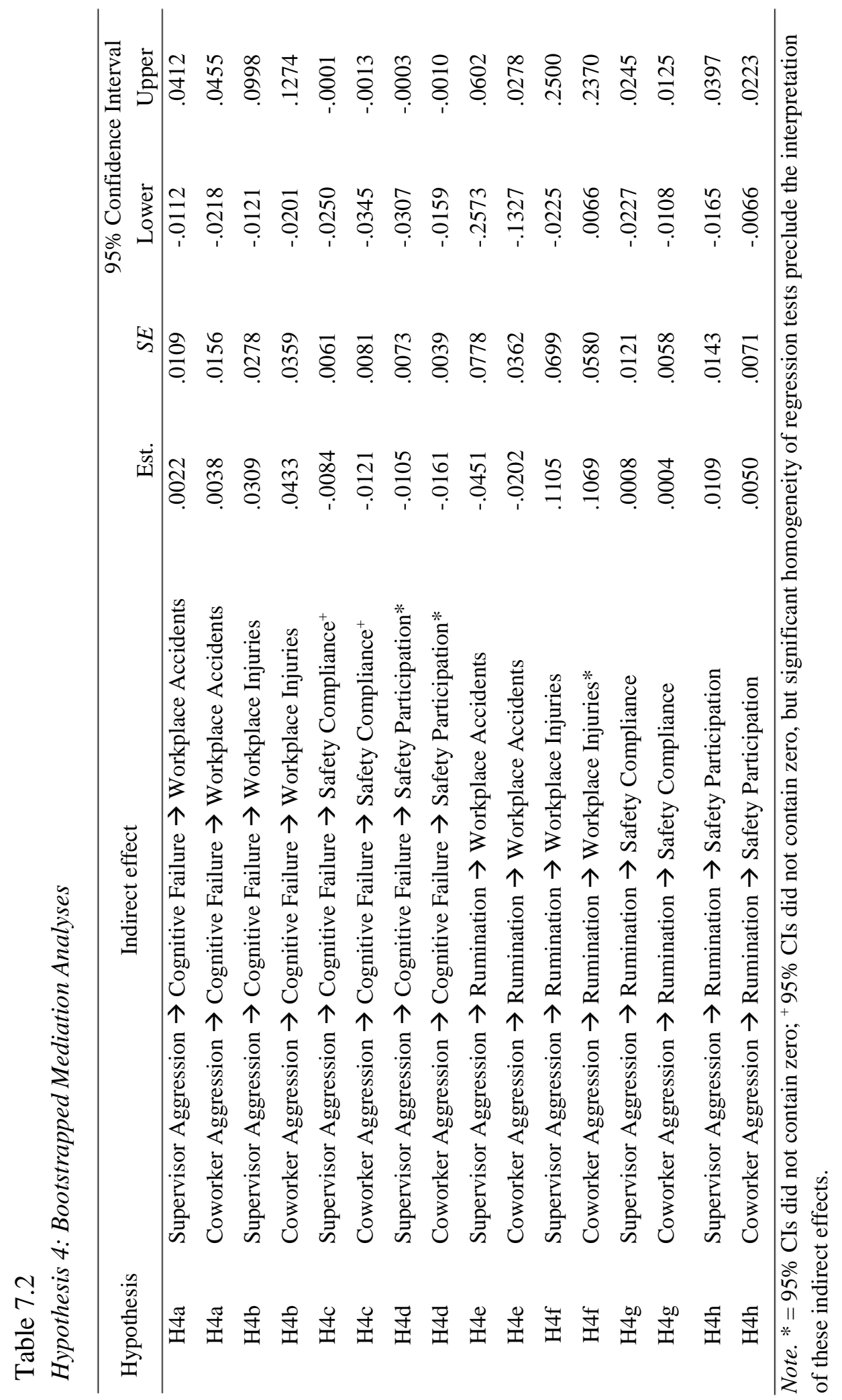


Hypotheses 5-6: Moderation. Hypothesis 5 proposed that mindfulness would moderate the relationship between workplace aggression and cognitive failure and rumination, and further, that safety climate would moderate the relationship between cognitive failure and rumination and workplace safety outcomes. No support was found for Hypotheses 5a-b, as mindfulness did not moderate the relationship between supervisor aggression and cognitive failure $\left(\beta=.01, t=.29, p=.77, \Delta R^{2}=.00\right)$ or rumination $\left(\beta=-.03, t=-.68, p=.50, \Delta R^{2}=.001\right)$, or the relationship between coworker aggression and cognitive failure $\left(\beta=.01, t=-.16, p=.89, \Delta R^{2}=.00\right)$ or rumination $(\beta=$ $\left..01, t=.31, p=.75, \Delta R^{2}=.00\right)$. Further, safety climate did not moderate the relationship between cognitive failure and workplace accidents $(\mathrm{B}=.004, \mathrm{SE}=.09, p=.96)$, workplace injuries $(\mathrm{B}=.11, \mathrm{SE}=.13, p=.41)$, safety compliance $(\beta=-.06, t=-1.45, p$ $\left.=.15, \Delta R^{2}=.003\right)$, or safety participation $\left(\beta=-.07, t=-1.62, p=.11, \Delta R^{2}=.004\right)$, failing to support Hypotheses 6a-d.

Safety climate was a marginally significant moderator of the relationship between rumination and workplace accidents $(\mathrm{B}=.32, \mathrm{SE}=.16, p=.055)^{6}$, and a significant moderator of the relationship between rumination and safety compliance $(\beta=-.14, t=-$ $\left.3.53, p<.001, \Delta R^{2}=.02\right)$, and safety participation $\left(\beta=-.15, t=-3.63, p<.001, \Delta R^{2}=\right.$ .02). A simple slopes test examining the moderating role of safety climate on the relationship between rumination and safety compliance was significant at both one

\footnotetext{
${ }^{6}$ Safety climate was a significant moderator of the relationship between rumination and workplace accidents $(\mathrm{B}=.36$, S.E. $=.16, p=.028)$ when the control variables (gender, supervisor status, and hours worked per week) were removed from the analyses.
} 
standard deviation above $(t=-2.71, p=.007)$ and below the mean $(t=-2.71, p=.008)$, providing support for Hypothesis $6 \mathrm{~g}$. Similarly, a simple slopes test examining the moderating role of safety climate on the relationship between rumination and safety participation was significant at both one standard deviation above $(t=-2.97, p=.003)$ and below the mean $(t=-2.83, p=.005)$, providing support for Hypothesis 6 h. Finally, safety climate did not moderate the relationship between rumination and workplace injuries $(\mathrm{B}=-.06, \mathrm{SE}=.14, p=.94)$, failing to support Hypothesis 6f. Significant interactions were graphed and can be seen in Figures 7.3-7.5. The scales on the y-axis of Figures $7.3-7.5$ were chosen to reflect the subset of the scale to which the majority of respondents replied (i.e., for safety compliance, $98.3 \%$ of the sample reported mean safety compliance levels of greater than 2 on a 5-point scale).

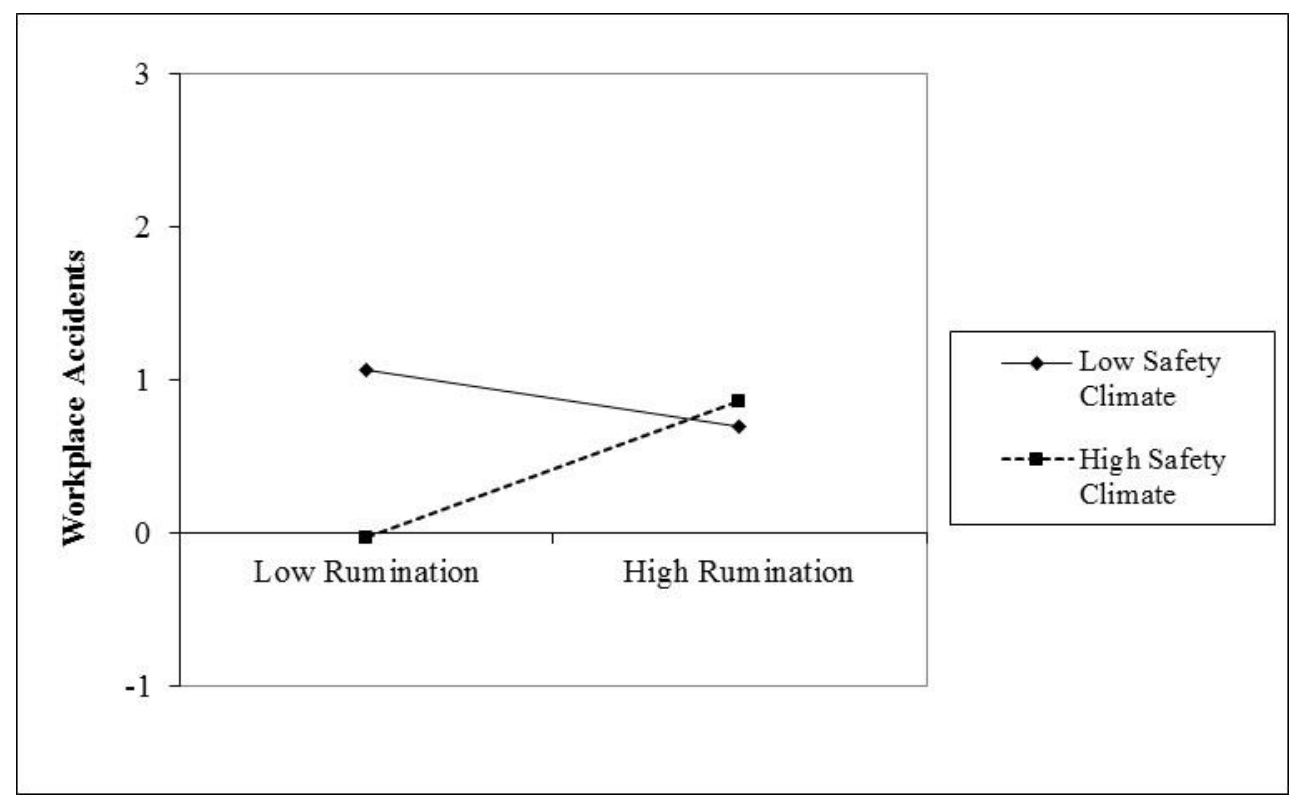

Figure 7.3. Hypothesis 6e. The relationship between rumination and workplace accidents moderated by safety climate. Note: The scale of 0-3 was selected as only $11 \%$ of participants reported experiencing more than one workplace accident in the previous six months (and only $3.6 \%$ reported experiencing more than 3 ). 


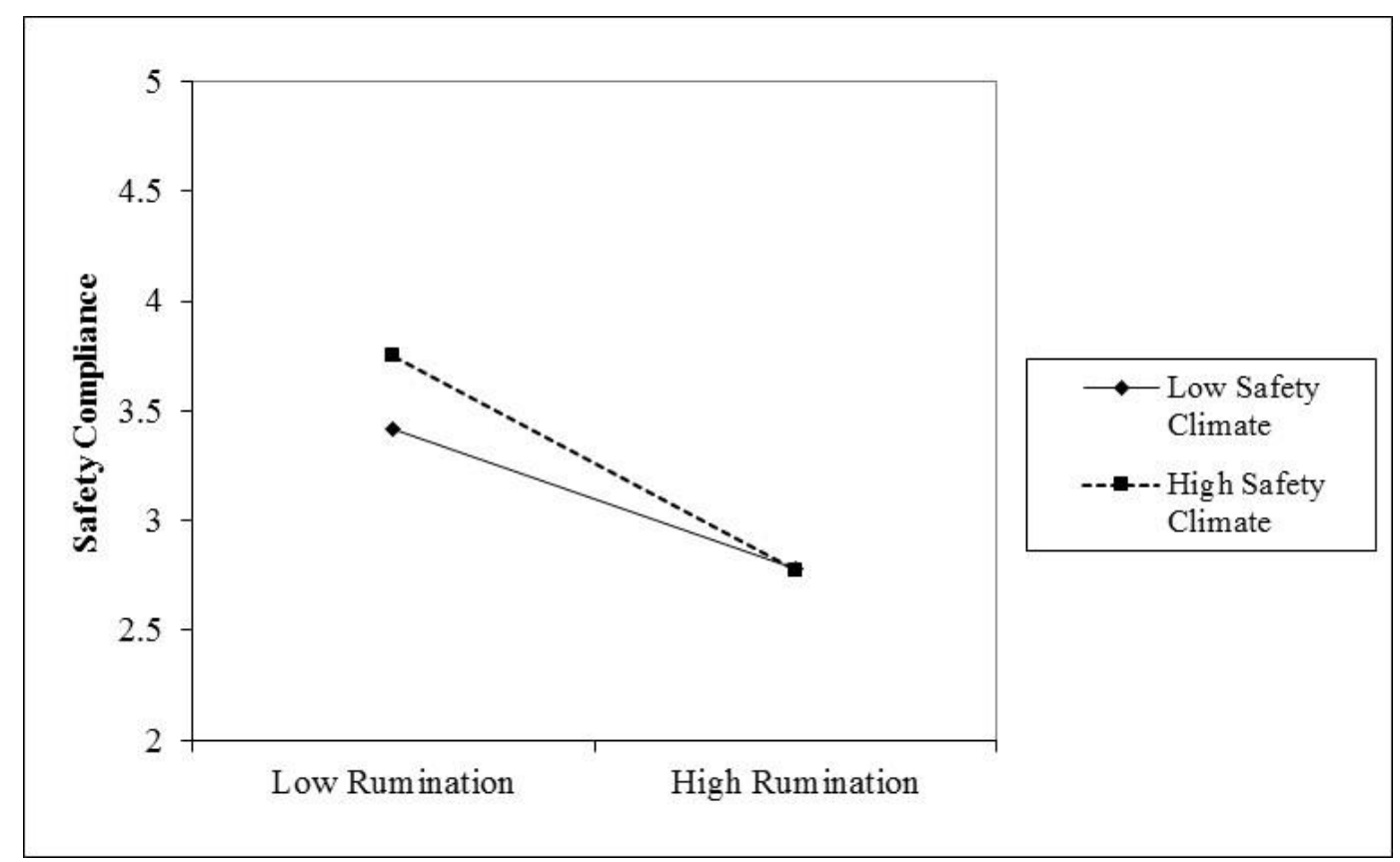

Figure 7.4. Hypothesis $6 \mathrm{~g}$. The relationship between rumination and safety compliance moderated by safety climate. Note: The scale shown on this graph was chosen as only $1.7 \%$ of the sample reported levels of safety compliance of 2 or lower on a 5-point scale.

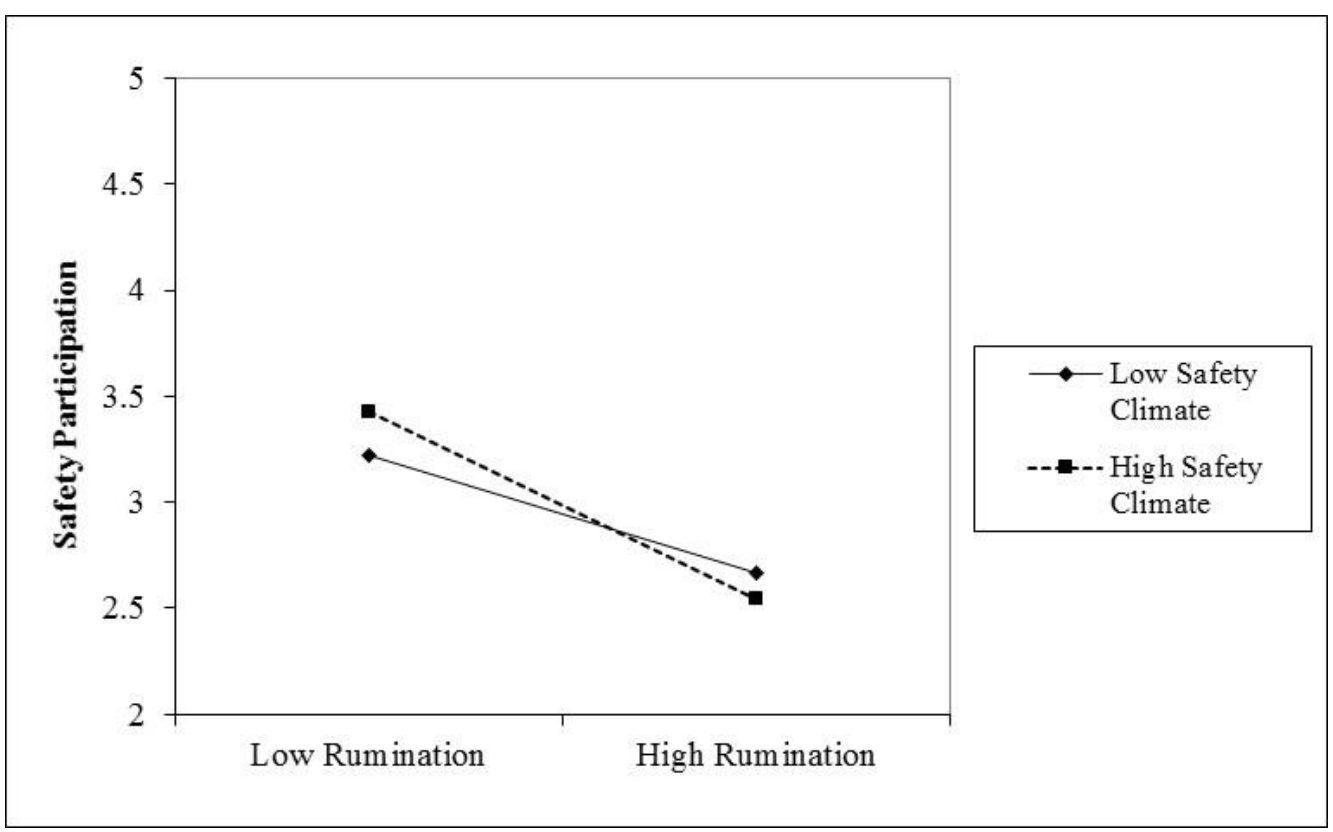

Figure 7.5. Hypothesis 6h: The relationship between rumination and safety participation moderated by safety climate. Note: The scale of $2-5$ was selected as only $2.4 \%$ of the sample reported safety participation levels of 2 or lower on a 5-point scale. 
Hypotheses 7-8: Moderated mediation. Hypotheses 7 and 8 proposed a moderated mediation model, also known as conditional indirect effects. This model is similar to that described by Preacher, Rucker, and Hayes (2007) as Model 5, with the exception that two distinct moderators were proposed to moderate the first and second stage of the mediation (mindfulness and safety climate, respectively). Mplus Version 6.0 (Muthén \& Muthén, 1998-2010) was used to analyze the moderated mediation model. Due to convergence issues with multi-level models, only the single level model results are reported. Specifically, the multi-level moderated mediation models examining workplace accidents, injuries, and safety participation would not converge due to a nonpositive definite Fisher information matrix, while the moderated mediation model examining safety compliance as an outcome demonstrated incomplete convergence, possibly due to singularity of the random effect. This may in part be due to the low intraclass correlation coefficients among all of the predictor variables.

The results provided limited support for Hypotheses 7-8 (complete results are available from the first author upon request). Conditional indirect effects were tested at one standard deviation above and below each moderator, as well as at the mean of each moderator. A pattern of results emerged, suggesting that the indirect effect of workplace aggression on safety behaviors via rumination was conditional on safety climate. For the purposes of these analyses, 90\% confidence intervals (CIs) were examined. Examining the relationship between workplace aggression and safety compliance, a statistically significant interaction between rumination and safety climate was found for coworker aggression, which implies that the indirect effect of coworker aggression on safety compliance via rumination is conditional on safety climate. Specifically, the $90 \% \mathrm{CI}$ did 
not include zero at one standard deviation below the mean of safety climate (Est. $=.003$, $\mathrm{SE}=.003$, CI: .001, .011), however it did at the mean level of safety climate $($ Est. $=.000$, $\mathrm{SE}=.001, \mathrm{CI}:-.002, .001)$, and at one standard deviation above the mean of safety climate $($ Est. $=.000, \mathrm{SE}=.003, \mathrm{CI}:-.005, .004)$. For supervisor aggression, however, the 90\% CI did include zero at one standard deviation below the mean of safety climate (Est. $=.005, \mathrm{SE}=.004, \mathrm{CI}: .000, .013)$, at the mean level of safety climate $($ Est. $=-.001, \mathrm{SE}=$ $.001, \mathrm{CI}:-.004, .001)$, and at one standard deviation above the mean of safety climate (Est. $=-.003, \mathrm{SE}=.003, \mathrm{CI}:-.009, .000)$. Taken together, this pattern of findings indicates that the indirect effect of coworker aggression on safety compliance via rumination is only significant for employees who report a low level of safety climate, and not for those who report average or greater than average levels of safety climate. Additionally, this indirect effect does not appear to hold for supervisor aggression.

Examining safety participation as the dependent variable, rumination also significantly interacted with safety climate. Regarding the relationship between supervisor aggression and safety participation, the $90 \%$ CI did not include zero at one standard deviation below the mean of safety climate $($ Est. $=.008, \mathrm{SE}=.005, \mathrm{CI}: .002$, $.017)$, though it did at the mean level of safety climate (Est. $=.001, \mathrm{SE}=.002$, CI: -.002 , $.004)$, and at one standard deviation above the mean of safety climate (Est. $=-.002, \mathrm{SE}=$ .002 , CI: $-.007, .000)$. Examining coworker aggression as a predictor, the $90 \%$ CIs did include zero at both \pm 1 standard deviation of safety climate $(+1 \mathrm{SD}$ : Est. $=.000, \mathrm{SE}=$ .002$, CI: $\{-.005, .003\} ;-1$ SD Est. $=.004, \mathrm{SE}=.003, \mathrm{CI}:\{.000, .012\})$, as well as at the mean level of safety climate (Est. $=.000, \mathrm{SE}=.001, \mathrm{CI}:-.001, .002)$. Interpreted as a whole, this pattern of findings indicates that the indirect effect of supervisor aggression 
on safety participation via rumination is only significant for employees who report a low level of safety climate, and not for those who report average or greater than average levels of safety climate. Additionally, this indirect effect does not appear to hold for coworker aggression. These findings provide partial support for Hypotheses $8 \mathrm{~g}-\mathrm{h}$. No support was found for indirect effects conditional on mindfulness, failing to support Hypothesis 7.

Overall, this pattern of results suggests that the indirect effect of workplace aggression on safety compliance and participation via rumination is conditional on the levels of perceived safety climate, with the interpretation that this indirect effect is significant for individuals with low perceptions of safety climate. Interestingly, the conditional indirect effect of workplace aggression on safety behaviors is also dependent on the source of aggression. While supervisor aggression indirectly affects safety participation via rumination, coworker aggression indirectly affects safety compliance via rumination. Both of these indirect effects are conditional on safety climate. A summary of the findings of this study can be found in Table 7.3. 


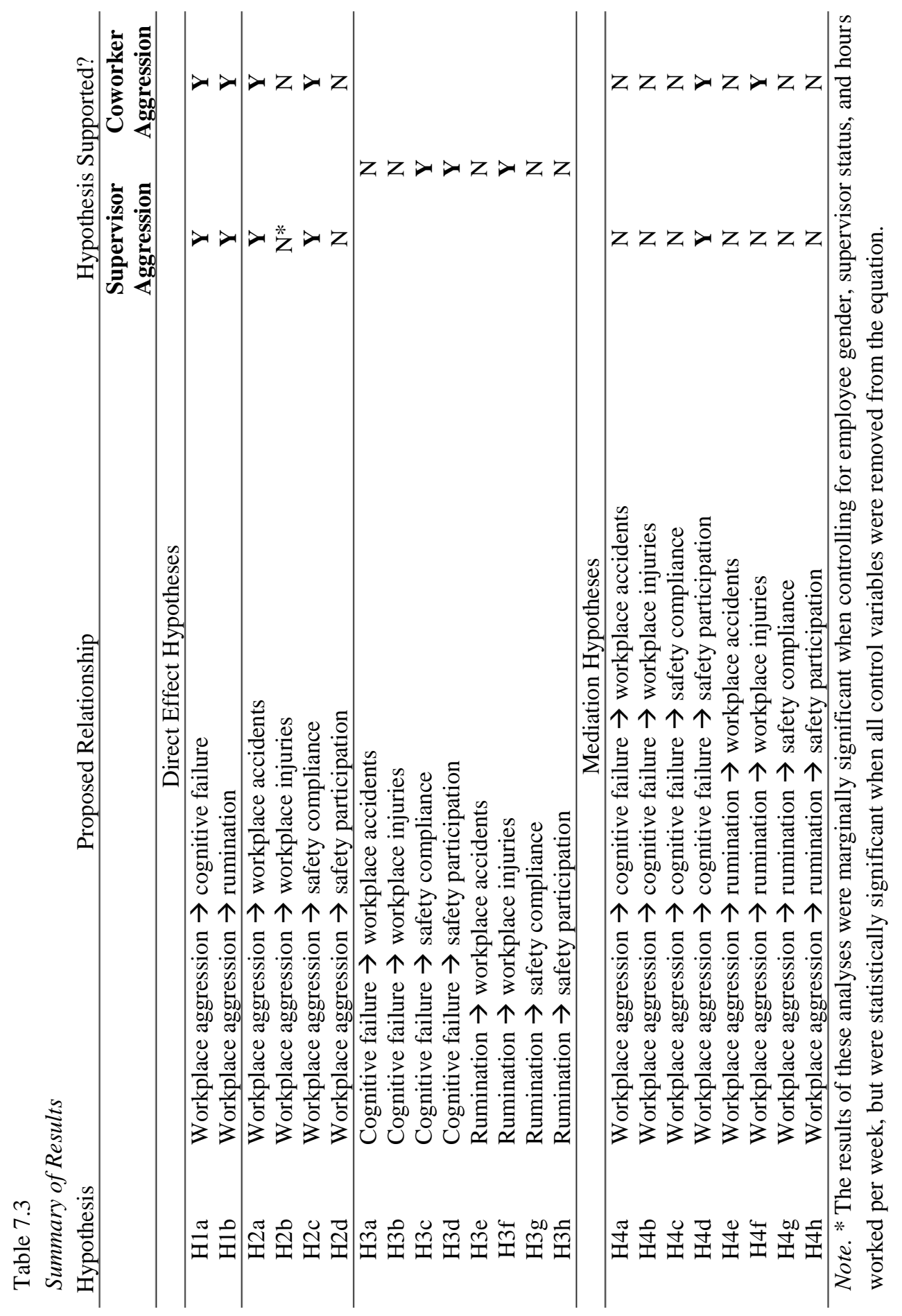




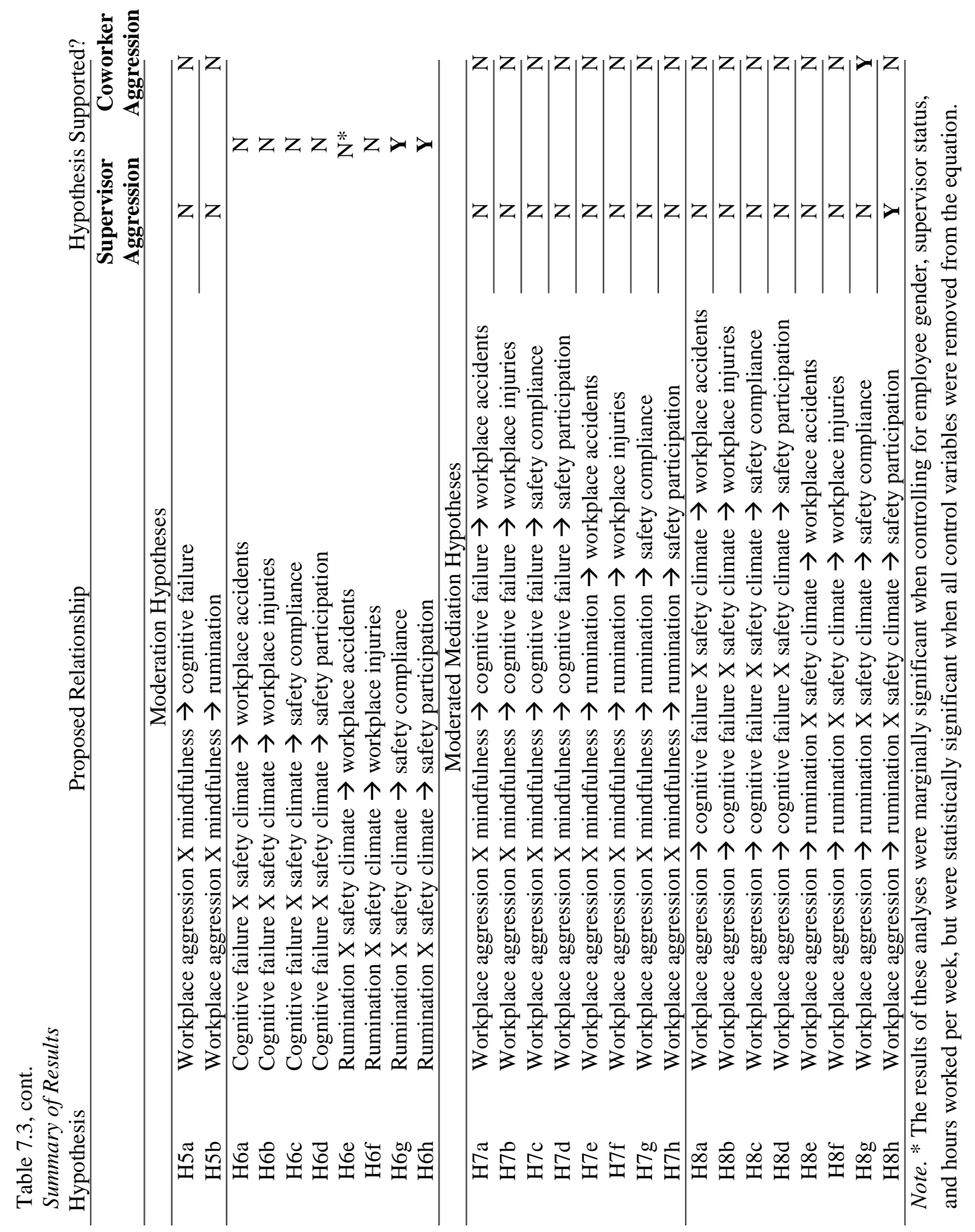




\section{Discussion}

Supervisor and coworker aggression were found to be directly associated with increased cognitive failure and rumination, as well as workplace accidents and reduced safety compliance. Cognitive failure was found to be negatively associated with safety compliance and safety participation, while rumination was positively associated with workplace injuries. Both supervisor and coworker aggression were indirectly associated with safety participation via cognitive failure, and coworker aggression was indirectly associated with workplace injuries via rumination. Additionally, cognitive failure was found to moderate the relationship between coworker aggression and safety compliance. Safety compliance was lower under conditions of high cognitive failure, and lowest under conditions of high coworker aggression.

Safety climate was found to moderate the relationship between rumination and workplace accidents, safety compliance, and safety participation. At low levels of rumination, workplace accidents were approximately one unit less under conditions of high safety climate than under conditions of low safety climate. Under high levels of rumination, the number of workplace accidents was similar, regardless of the level of reported safety climate. For both safety compliance and participation, under conditions of high rumination, safety behaviors were lower, regardless of the level of safety climate. However, under conditions of low rumination, safety behaviors were higher when perceived safety climate was also high. Again, though not necessarily intuitive, there are possible explanations for this finding. Whereas safety climate was proposed to buffer against the negative effects of rumination on workplace safety, it may be that employees do not as readily pick up on environmental indicators of safety climate while 
experiencing high levels of rumination. It may instead be more useful to keep levels of safety climate high while also seeking to identify ways to reduce work-related rumination; for example, by encouraging employees to engage in nonwork recovery experiences (Fritz, Ellis, Demsky, Guros, \& Lin, 2013).

Finally, a pattern of conditional indirect effects was identified, in which the relationship between workplace aggression and safety behaviors via rumination was conditional on low levels of perceived safety climate. Specifically, the indirect effect of supervisor aggression and safety participation via rumination was conditional on low levels of safety climate, while the indirect effect of coworker aggression on safety compliance via rumination was conditional on low levels of safety climate. In general, this suggests that workplace aggression is negatively associated with safety behaviors via rumination only for those employees who also report low levels of safety climate. This process does not appear to occur for employees who report average or high levels of workplace safety climate. The differential findings identified for supervisor and coworker aggression in relation to safety behaviors is in line with previous research on workplace aggression, which suggests that both supervisor and coworker aggression have stronger effects on attitudinal and behavioral outcomes than outsider aggression (Hershcovis \& Barling, 2010), though to date, little is known about the potential differential effects of the source of workplace aggression on measures of task versus contextual performance.

Taken together, while the hypotheses in the current study were only partially supported, mindfulness and safety climate, both conceptualized as resources, were found to be associated in the expected directions with resource depletion and workplace safety. Further, the process through which workplace aggression may indirectly influence 
workplace safety behaviors via resource depletion appeared to occur particularly for individuals reporting low levels of perceived safety climate. Overall, this study provides several important implications, both theoretical and practical, for examining the associations between workplace aggression, resources, and workplace safety.

\section{Theoretical Implications}

In line with both Conservation of Resources (COR; Hobfoll, 1989) theory and the Job Demands-Resources Model (Demerouti et al., 2001), workplace aggression was found to be positively associated with both cognitive failure and rumination, which we conceptualized in the current study as a depletion of resources. On the other hand, limited support was found for the buffering effects of both mindfulness and safety climate, contradicting the theories from which these hypotheses were derived. While this limited support included the relationship between rumination and safety behaviors, no moderating effects between cognitive failure and workplace safety were found. It may be the case that employees experiencing cognitive failure are also unable to perceive social context clues indicating high levels of safety climate, which might otherwise reduce their exposure to workplace accidents and injuries, or increase their safety behaviors.

Additionally, a significant conditional indirect effect between supervisor and coworker aggression and safety behaviors via rumination was found under conditions of low levels of perceived safety climate. However, this indirect effect was not significant under high or average levels of perceived safety climate, which is in line with COR theory and the JD-R Model. COR theory would suggest that employees low in organizational resources (i.e., safety climate) may be less likely to invest additional resources into work-related behaviors such as safety compliance and participation. In 
support of the JD-R model, which proposes that work-related resources may buffer the effects of job demands, our results suggest that a deficit of organizational resources fails to buffer the effects of work-related rumination on safety behaviors.

\section{Practical Implications}

While the findings of the current study should be interpreted with caution, there are still several implications that can be drawn. Workplace aggression - both supervisorand coworker-initiated - were found to be positively associated with workplace accidents and negatively associated with safety compliance behaviors. These findings are in line with previous limited research on workplace aggression and workplace safety outcomes (e.g., Yang, 2009). Additionally, low levels of safety climate were found to moderate the indirect effect of workplace aggression on safety behaviors via rumination. While much of the literature around workplace aggression focuses on the negative outcomes associated with experienced aggression, the current study provides information on a potential organizational resource that may also play a role in weakening the association between workplace aggression and safety behaviors. This is particularly important as research has demonstrated that safety climate is susceptible to change via organizational interventions (e.g., Zohar \& Luria, 2003; Zohar \& Polachek, 2014).

Though effect sizes were small, particularly when considering the moderating role of safety climate, even small changes in safety climate may have practical significance for organizations and employees alike. To illustrate this point, in the United States in 2007 there were more than 5,600 fatal and 8,559,000 nonfatal occupational injuries at a cost of $\$ 6$ billion and $\$ 186$ billion, respectively (Leigh, 2011). The total estimated costs of occupational injuries and illnesses combined was $\$ 250$ billion, with injuries 
comprising $77 \%$ of this total cost. Though these costs may seem overwhelming in scope, it is possible that even small changes to improve safety climate and performance within an organization may contribute to improving safety outcomes such as those listed above.

In addition to safety climate, mindfulness was also examined as a potential resource that could mitigate the negative associations between workplace aggression, resource depletion, and workplace safety. While no support was found for mindfulness as a moderator of these relationships, it was associated with reduced resource depletion and increased safety climate, safety compliance, and safety participation. While future research should explore these associations in more detail, these findings contribute to the existing body of literature on the benefits of mindfulness, which support the use of mindfulness-based stress reduction intervention programs in an effort to improve individual health and well-being (Grossman et al., 2004).

Though it is worthwhile to identify potential resources that may diminish the negative effects of workplace aggression, it is also critical that organizations take proactive measures to reduce the occurrence of workplace aggression. Limited intervention work has been done in this realm, but researchers have provided a number of recommendations in this regard, including setting zero tolerance policies, conducting exit interviews, and believing and supporting employees who lodge complaints regarding workplace aggression (Porath \& Pearson, 2010). Further, a recent review of mistreatment climate indicated that it would be useful to target future intervention work towards organizational climate perceptions, particularly in increasing perceptions of civility climate (Yang, Caughlin, Gazica, Truxillo, \& Spector, 2014). 


\section{Potential Limitations \& Future Directions}

It is important to interpret the current findings in light of study limitations. The current study is cross-sectional, therefore limiting causal inferences. However, the proposed model still provides useful insights into the potential relationship between workplace aggression and workplace safety, which has thus far received limited attention. Researchers should consider using time-lagged longitudinal designs, in which all variables of interest are measured at multiple points in time. Additionally, while the current study assessed all variables via the use of self-report instruments, it would be worthwhile to collect other-reports of key study variables. For example, either observer reports of safety behaviors or administrative data on accidents and injuries would be viable avenues for examining workplace safety outcomes. Regarding measurement, the workplace cognitive failure scale used in the current study displayed less than ideal levels of reliability, which may indicate bias within the measurement of this construct. It may be that the items used in this particular scale were not as applicable to the occupations under examination in the current study, or that other indicators of this construct within this population were not measured by the current scale.

To date, limited research has examined the buffering impact of resources on the relationship between workplace aggression and its outcomes (Schat \& Kelloway, 2003). While mindfulness was not found to be a moderator of the relationship between workplace aggression and resource depletion, perceived safety climate was found to moderate the relationship between rumination and safety behaviors. It may be worthwhile to examine mindfulness as a moderator between workplace aggression and health outcomes, as engaging in mindfulness practices may reduce the negative activation that is 
inherently associated with the experience of workplace aggression (Grossman et al., 2004).

Partial support was found for the role of resource depletion as a mechanism through which workplace aggression is associated with safety behaviors. This effect was found to be conditional on the level of safety climate reported by employees, such that workplace aggression is only associated with safety behaviors via increased rumination for employees reporting a low level of safety climate. Whereas the resources examined in the current study were selected for their relevance to workplace safety, there are a number of resources that have been examined through the lens of Conservation of Resources theory (Halbesleben, Neveu, Paustian-Underdahl, \& Westman, 2014) which may be worth exploring in conjunction with workplace aggression. For example, these resources include core self-evaluations (Judge \& Bono, 2001), self-esteem (Vinokur \& Schul, 2002), and locus of control (Vinokur \& Schul, 2002), as well as civility climate (Osatuke, Moore, Ward, Dyrenforth, \& Belton, 2009).

\section{Conclusion}

The current study demonstrated significant relationships between both supervisor and coworker aggression and resource depletion, as well as workplace accidents and safety behaviors. Broadly, a pattern of results emerged which suggests that the indirect effect of workplace aggression on safety behaviors via rumination was conditional on perceived safety climate, such that this indirect effect is significant for individuals who report low levels of safety climate. While safety climate is a known predictor of safety behaviors, these additional findings highlight the potential for safety climate to protect 
employees and organizations against workplace stressors (e.g., workplace aggression) that may diminish employees' resources, in turn negatively influencing safety outcomes. 


\section{Chapter 8: General Discussion}

The above three studies shed light on potential outcomes of workplace aggression, including under-examined outcomes, the mechanisms through which workplace aggression influences these outcomes, and potential moderators of this process. Specifically, psychological detachment, perceived stress, cognitive failure, and rumination were examined as mechanisms through which workplace aggression was associated with outcomes such as work-family conflict (WFC), parenting behaviors, and workplace safety. Additionally, both mindfulness and safety climate were examined as potential resources that could alleviate the negative associations between workplace aggression and employee outcomes.

\section{Statement of Purpose Revisited}

This body of research was motivated by three related research questions: 1) Does psychological detachment from work serve as a potential mechanism through which workplace aggression is associated with increased work-family conflict? 2) Does workplace aggression spill over to the nonwork domain to impact parent-child interactions? and 3) Does workplace aggression influence safety in the workplace? The preceding manuscripts examine these research questions in detail, and provide several answers and potential directions for future research.

In Study 1 (Chapter 3), a multi-source, cross-sectional dataset was used to examine the hypothesis that coworker reports of workplace aggression are indirectly associated with self- and significant-other reports of WFC via decreases in self-reported psychological detachment from work (Demsky, Ellis, \& Fritz, 2014). Overall, support was found for the hypothesized model, suggesting that workplace aggression may spill 
over to negatively influence employees' nonwork experiences. Workplace aggression was associated with decreases in psychological detachment from work, which in turn was associated with increases in WFC. These findings suggest that it may be most important to make an effort to psychologically detach from work on days in which it is most difficult. Based on this first study, psychological detachment is indeed one potential mechanism through which workplace aggression is associated with increased workfamily conflict.

Study 2 (Chapter 5) continues the examination of the effects of workplace aggression on nonwork outcomes. While earlier research has connected workplace aggression to employee and spouse-reported outcomes in the nonwork domain (Carlson, Ferguson, Hunter, \& Whitten, 2012; Restubog, Scott, and Zagenczyk, 2011), no studies to my knowledge have examined the association between workplace aggression and parenting behavior. Extending what is known of workplace aggression's negative association with WFC and spousal relationships (Story \& Repetti, 2006), it is likely that an employee's experience of workplace aggression may also negatively influence interactions with children after work. The second study of this dissertation sought to empirically examine this hypothesis utilizing a longitudinal sample of long term healthcare employees (Demsky \& Hammer, in preparation). According to our findings, experiences of psychological and physical workplace aggression negatively influenced parental warmth twelve months later via baseline reports of perceived stress, even after controlling for baseline levels of parental warmth. While this relationship was statistically significant, there was no indirect effect found between workplace aggression and twelve month reports of child disclosure. This study contributes to the literature which examines 
nonwork outcomes of workplace aggression, as well as previous work that has connected parents' work experiences with parenting behaviors at home (Bass, Butler, Grzywacz, \& Linney, 2009; Bumpus, Crouter, \& McHale, 1999; Cho \& Ciancetta, 2015; Repetti \& Wood, 1997). Addressing this dissertation's second research question, it appears that workplace aggression may indeed have significant downstream effects on employees' interactions with family members, even up to a year after the initial reports of aggression.

Finally, Study 3 (Chapter 7) sought to identify potential resources that may mitigate the negative outcomes of workplace aggression on workplace safety (Demsky \& Fritz, in preparation). Workplace aggression was proposed to be negatively associated with workplace safety (i.e., increased accidents and injuries, and decreased safety behaviors) via resource depletion (i.e., increased cognitive failure and rumination). Further, mindfulness was proposed to moderate the first stage of this mediation process, while safety climate was proposed to moderate the second stage. Overall, partial support was found for the hypothesized relationships, such that workplace aggression was associated with increased resource depletion and workplace accidents, and decreased safety compliance. Cognitive failure was in turn negatively associated with both safety compliance and safety participation, and rumination was positively associated with workplace injuries. In terms of buffering relationships, safety climate was found to moderate the relationship between rumination and safety compliance and participation, such that safety climate buffered against the negative effects of workplace aggression only under low levels of rumination. Finally, partial support was found for the overall proposed model, such that the indirect effect of workplace aggression on safety compliance and participation via rumination was significant only for employees who 
reported a low level of safety climate. These findings address the third group of research questions motivating this body of research by illuminating the process through which workplace aggression is associated with workplace safety, as well as identifying two potential moderators of this process.

\section{Theoretical Implications}

Three theoretical frameworks were used in the development of this dissertation: Conservation of Resources Theory (COR; Hobfoll, 1989), the Work-Home Resources Model (ten Brummelhuis \& Bakker, 2012), and the Job Demands-Resources Model (Demerouti \& Bakker, 2011). Overall, support was found for these theories, though future research may highlight additional aspects of these theories in relation to workplace aggression.

Conservation of resources (COR) theory. COR theory motivated both the first and third studies in this body of research. Workplace aggression can be conceptualized as a threat to one's resources, which in turn results in higher levels of stress and decreased resources. Ongoing exposure to resource loss may in turn be associated with resource loss spirals (Hobfoll, 1998, 2001). In the first study, workplace aggression was associated with increased WFC via reduced psychological detachment from work. In line with COR theory, employees experiencing workplace aggression may be deficient in resources that could otherwise be invested in resource-gaining activities, such as recovery experiences (Sonnentag \& Fritz, 2007).

In the third study, workplace aggression was found to be positively associated with resource depletion (i.e., cognitive failure and rumination), in line with the conceptualization of workplace aggression as a threat to employees' resources. 
Additionally, workplace aggression was associated with reduced safety compliance and increased accidents, which suggests that employees may have reduced resources available for investment in safety-related behaviors. Mindfulness and safety climate were proposed as personal and organizational resources, respectively, that employees may draw on to counteract the negative effects of workplace aggression. While no moderating support was found for mindfulness, mindfulness was associated in the expected direction with resource depletion. Further, safety climate was found to be a significant moderator of the resource depletion and safety behavior association. In this way, the provision of resources may be seen as beneficial to employees, which is in line with the tenets of COR theory.

While this dissertation primarily focused on resource loss, it would be worthwhile to examine the likelihood that resource gain spirals may be negatively associated with employees' exposure to workplace aggression, or may be able to buffer the negative effects of workplace aggression. Additionally, it would be beneficial to further examine the proposition that workplace aggression threatens employees' resources, by empirically examining workplace aggression's association with the resources recently reviewed by Halbesleben, Neveu, Paustian-Underdahl, and Westman (2014).

Work-home resources model. The Work-Home Resources Model was proposed as an extension of COR theory (ten Brummelhuis \& Bakker, 2012), and suggests that researchers should explicitly examine the mechanisms through which work demands transfer from the work domain to the nonwork domain and vice versa, as well as the means through which resources transfer between domains. Though relevant to each of the enclosed studies, this theory was explicitly drawn upon in the second study, in which 
workplace aggression was found to indirectly affect parenting behaviors one year later via the mechanism of increased employee perceived stress. Perceived stress was conceptualized as an indicator of resource loss, as COR theory states that stress is a result of a threat to one's resources, a loss of resources, or a failure to gain resources after an investment of resources (Hobfoll, 1989). The findings of this study also indirectly suggest the presence of a resource loss spiral, such that workplace aggression is associated with initial resource loss (i.e., perceived stress), which is in turn associated with decreased parental warmth one year later, which is likely to threaten employees' relationships with children and other family members.

The study described above directly tests a proposition of the Work-Home Resources model, and finds support for the underlying process through which work demands transfer to the home domain. However, it would be worthwhile to test specific resources that may be lost due to workplace aggression exposure (e.g., positive affect), as well as examining the process through which resources in the work domain transfer to the nonwork domain, or vice versa (e.g., social support, workplace flexibility, etc.).

Job demands-resources (JD-R) model. Finally, the third study was also motivated by the JD-R Model (Demerouti et al., 2001). Workplace aggression was conceptualized as a job demand, as it is an aspect of the job that requires sustained psychological effort to manage, and is in turn associated with certain physiological and/or psychological costs (Demerouti \& Bakker, 2011). As described above, workplace aggression was indeed found to be associated with indicators of resource depletion and workplace safety, which supports the JD-R model. Additionally, mindfulness and safety climate were conceptualized as personal and organizational resources, as they are aspects 
of the person and organization that can help to reduce the impact of job demands or serve a purpose in accomplishing work goals. Although each was proposed as a moderator of the negative effects of workplace aggression on resource depletion and workplace safety, more support was found for direct effects of these resources, which is in line with previous research on the JD-R model (Bakker \& Demerouti, 2007).

In line with prior reviews of the JD-R model, additional work should be done to examine this theory within a multi-level context, which may be possible utilizing data from the third study of this dissertation (Demerouti \& Bakker, 2011). Additionally, it would still be a useful contribution to examine the direct effects of these hypothesized resources, however it may also be worthwhile to consider other organizational and personal resources that may buffer the negative effects of workplace aggression.

\section{Practical Implications}

Overall, this multi-study examination of workplace aggression provides a number of potential implications for both employees and organizations. These implications may be thought of as either preventative or reactive measures. While it would be preferable to prevent the occurrence of workplace aggression in the first place, the high incidence rate of workplace aggression suggests that employees and organizations alike should also have strategies in place to react to and reduce the negative effects of workplace aggression.

Workplace interventions. The three studies in this body of work suggest a number of possible workplace interventions that may be ideal for reducing the negative associations between workplace aggression and employee well-being. In the first study, a lack of psychological detachment is identified as a mechanism through which workplace 
aggression is associated with increased work-family conflict (Demsky, Ellis, \& Fritz, 2014). Research has suggested that recovery experiences are trainable, and that employees benefit from such training programs (Hahn, Binnewies, Sonnentag, \& Mojza, 2011; Siu, Cooper, \& Phillips, 2013). Providing employees with training on recovery experiences may weaken the association between workplace aggression and work-family conflict, in addition to promoting other forms of employee well-being (e.g., reduced burnout, increased recovery self-efficacy).

In addition to training on recovery experiences, providing general stress management trainings to employees may also weaken the negative spillover process between work and nonwork domains. In the second study, perceived stress was found to be a mechanism through which workplace aggression indirectly influenced parental behaviors (i.e., parental warmth) one year later. By assisting employees in managing work-related stress, organizations may prevent work demands from spilling over into the nonwork domain to negatively influence employees' interactions with their families. As research on the work-family interface has indicated that family demands conversely spill over into the work domain, organizations may indeed reap benefits by reducing workrelated strain in their employees' family domains (Amstad, Meier, Fasel, Elfering, \& Semmer, 2011). Existing workplace interventions that have been shown to reduce the negative effects of work demands on employee health and well-being include training aimed at increasing family supportive supervisor behaviors (FSSBs; Barbosa, Bray, Brockwood, \& Reeves, 2014; Hammer, Demsky, Kossek, \& Bray, 2015) and mindfulness practices (Grossman, Niemann, Schmidt, \& Walach, 2004). 
Finally, the third study examines additional resources that may be worthwhile to target via workplace interventions. Safety climate (Zohar, 1980) was found to be a moderator of the relationship between rumination and safety behaviors, such that safety behaviors were highest under conditions of low rumination and high safety climate. Research has indicated that safety climate is a valid and reliable predictor of workplace safety (e.g., Clark, 2006; Neal \& Griffin, 2006), and is also susceptible to influence via workplace trainings (Zohar \& Polachek, 2014). Mindfulness was also examined as a personal resource that may weaken the negative effects of workplace aggression. While no support was found for a moderating influence, mindfulness was associated with decreased levels of workplace aggression, cognitive failure, and rumination, and increased workplace safety (Demsky \& Fritz, in preparation). Although these findings should be replicated with a longitudinal or experimental study design, this work is in line with existing research on the benefits of mindfulness (Grossman, Niemann, Schmidt, \& Walach, 2004). Similar to the resources noted above, mindfulness has also been successfully increased via the use interventions (Kabat-Zinn, 1990).

Employee strategies. While the aforementioned strategies may be addressed via organizational strategies, employees may also act directly to address their own behavior in the workplace. For example, Hahn and colleagues (2011) tested a recovery intervention with a group of employed adults, and found beneficial effects on participants' reported recovery experiences. Seeking out recovery experiences during nonwork time (e.g., hobbies, physical activity, etc.) may be one potential way to reduce negative outcomes associated with work demands such as workplace aggression (Fritz, Ellis, Demsky, Lin, \& Guros, 2013). Making time to actively switch between work and 
nonwork domains may also prevent negative workplace experiences from spilling over into the nonwork context. For example, employees may choose to stop checking email after a certain time, or may listen to soothing music on the commute home to unwind from a stressful day at work.

Preventing workplace aggression. In addition to providing employees with the necessary tools to cope with workplace aggression, organizations should place an emphasis on the primary prevention of workplace aggression, in line with occupational health psychology principles (Quick, 1999). The prevalence of this phenomenon may suggest its inevitability; however, there are a number of steps employers can take to reduce the incidence rate of workplace aggression (Porath \& Pearson, 2010). For example, instituting zero tolerance policies, conducting exit interviews, and taking complaints seriously may help to deter workplace aggression. Organizations may also want to promote positive workplace behavior by instituting workplace civility trainings (Osatuke, Leiter, Belton, Dyrenforth, \& Ramsel, 2009).

\section{Potential Limitations}

The three studies described above offer a number of theoretical and practical implications; however, it is important to consider the limitations of this research as well. Specifically, concerns regarding study design and measurement should be taken into consideration. First, it is worth noting that both the first and third studies in this multimanuscript dissertation were cross-sectional in nature. This limits our ability to make causal inferences regarding hypothesized relationships. However, additional analyses were conducted in the first manuscript (Demsky et al., 2014) to address concerns of reverse causality. Further, in the first and third studies, both theory and prior empirical 
research support the directionality of the hypothesized relationships. Whereas the first and third studies were cross-sectional, the second study sought to overcome this limitation through the use of a longitudinal sample of long-term healthcare employees. In addition to contributing to the literature on work demands and parenting behaviors, this particular study is the first to our knowledge to examine the longitudinal associations between workplace aggression and parenting behaviors.

Second, both the second and third studies consisted of self-report data, which may raise concerns regarding common method variance (Podsakoff, MacKenzie, Lee, \& Podsakoff, 2003). However, as a main goal of this body of research was to uncover the underlying processes through which workplace aggression influences employee outcomes, it was necessary to collect data on these processes directly from employees. This is particularly true given that the processes we examine in the enclosed studies may be difficult to directly observe (i.e., psychological detachment, perceived stress, rumination, and cognitive failure). The first study utilizes a multi-source dataset to address some of the concerns regarding common method variance. Results indicate that our findings were also generalizable across both self and other-reports of workplace aggression.

Next, each of the preceding studies examines workplace aggression within a very specific occupational context. The first study examines workplace aggression in a sample of university employees, while the second utilizes a sample of long-term healthcare employees. The final study examines workplace aggression and occupational safety in the United States Department of Agriculture Forest Service. While both the first and third samples are comprised of nearly an equal amount of men and women, the second sample 
(i.e., long-term healthcare employees) is predominantly female. Each of these occupations consists of a unique set of demands and resources. For example, healthcare workers are subject to some of the highest rates of workplace aggression and violence (OSHA, 2015), which may be differentially associated with employee outcomes. We consistently find that workplace aggression is associated with negative employee outcomes across the three studies. Nevertheless, it would be useful to replicate these findings in a number of occupational contexts prior to generalizing more widely.

Finally, Hershcovis (2011) noted the importance of collecting data on the source from which workplace aggression originates. Previous research has indicated that workplace aggression may be associated with differential outcomes depending on the source, which has important implications for interpretation and practical recommendations (Hershcovis \& Barling, 2010). Whereas the first and second studies do not differentiate the source of aggression, the third study examines workplace aggression from both coworkers and supervisors. In general, findings of the third study indicate that the direct effects of workplace aggression on resource depletion and workplace safety tend to generalize across sources. However, the conditional indirect effects of workplace aggression on safety behaviors via rumination displayed differential results based on the source of aggression. These findings suggest possible avenues for future research on the association between workplace aggression and work performance.

\section{Future Research}

Although the current research offers a number of contributions to the literature surrounding workplace aggression, there are several additional areas that should be 
explored in further detail with future research. Several of these potential future directions are elaborated on below.

Longitudinal designs. Cross-sectional data necessarily limits our ability to make causal inferences regarding relationships amongst key variables. In the second study, effects of workplace aggression on the nonwork domain were found up to one year later. It would be worthwhile to examine other employee and organizational outcomes of workplace aggression over a longer time period, which would allow researchers to determine the extent to which employees continue to be affected by workplace aggression exposure. In addition to using longitudinal designs over months, or even years, it would be useful to examine workplace aggression via the use of day-level studies (e.g., Volmer, Binnewies, Sonnentag, \& Niessen, 2012). For example, in the second study, while workplace aggression was found to be indirectly associated with parental warmth one year later, no significant effects were found for child disclosure. It may be that these form of interactions are affected on a shorter time frame, which would potentially be identifiable over several days (or weeks) of data collection.

Objective health outcomes. Workplace aggression has been associated with a host of negative employee and organizational outcomes. Nevertheless, we know much less about the association between workplace aggression and objective health outcomes such as blood pressure, sleep, and cortisol. One of the underlying processes associated with workplace aggression (Study \#3), rumination, has previously been linked to higher levels of cortisol (Zoccola, Figueroa, Rabideau, Woody, \& Benencia, 2014). Examining such outcomes in future studies may help to triangulate existing findings on workplace aggression, as well as contribute to the business case for reducing workplace aggression. 
In addition to contributing to the literature on workplace aggression, the examination of objective health outcomes may also serve to further develop the theoretical frameworks commonly used in this literature. For example, Demerouti and Bakker (2011) recently made an explicit call for future research on the JD-R Model to incorporate objective health outcomes.

Workplace aggression interventions. To date, very little research has been conducted on interventions aimed at reducing the prevalence of workplace aggression. In a recent meta-analysis of mistreatment climate, it was noted that future intervention development work may seek to change the occurrence of workplace mistreatment by addressing the workplace climate concerning mistreatment (Yang, Caughlin, Gazica, Truxillo, \& Spector, 2014). However, Yang and colleagues (2014) indicated that interventions aimed at reducing workplace aggression tend to have stronger effects when focused on promoting a climate of civility as opposed to reducing or weakening mistreatment climate. Research has suggested that civility interventions can be useful means of increasing civil behavior in the workplace (Osatuke et al., 2013), and at least one intervention aimed at reducing workplace aggression in the healthcare context has also been developed (Yragui, Silverstein, Foley, Johnson, \& Demsky, 2012). It is critical that future research address the development of individual and organization-level interventions that may reduce the occurrence of workplace aggression, as well as the negative effects of workplace aggression.

Additional resources as moderators. The current body of research examined a very limited range of potential resources as buffers of the negative effects of workplace aggression - specifically, mindfulness and safety climate. Evidence was limited for these 
resources as moderators of this process. Additional resources may be worth exploring in future research on workplace aggression, and would be particularly relevant in developing interventions aimed at reducing the effects of workplace aggression. Research has identified organizational support (Schat \& Kelloway, 2003) and family supportive supervisor behaviors (Yragui, Hammer, \& Demsky, 2014) as moderators of the relationship between workplace aggression and employee well-being. Other resources worth exploring in relationship to workplace aggression may include resilience, emotional stability, job security, and time away from work (Halbesleben et al., 2014).

\section{Conclusion}

This dissertation examined the relationships between workplace aggression, nonwork outcomes, and workplace safety by shedding light on the mechanisms through which these relationships occur, as well as on potential resources that may mitigate the negative outcomes of workplace aggression. Workplace aggression was found to be positively associated with work-family conflict, and negatively associated with parental warmth and workplace safety, via the mechanisms of psychological detachment, perceived stress, cognitive failure, and rumination. Safety climate was identified as one potential organizational resource that buffers some of the negative effects of workplace aggression on safety behaviors via rumination. This body of research contributes to the literature on workplace aggression by illuminating the processes through which workplace aggression negatively influences employee outcomes. Additionally, this research offers several important practical implications, particularly in the development of workplace interventions and management practices. 


\section{References}

Abbe, O. O., Harvey, C. M., Ikuma, L. H., \& Aghazadeh, F. (2011). Modeling the relationship between occupational stressors, psychosocial/physical symptoms and injuries in the construction industry. International Journal of Industrial Ergonomics, 41, 106-117. doi: 10.1016/j.ergon.2010.12.002

Ahlberg-Hulten, G. K., Theorell, T., \& Sigala, F. (1995). Social support, job strain and musculoskeletal pain among female health care personnel. Scandinavian Journal of Work Environment \& Health, 21, 435-439.

Aiken, L. S. \& West, S. G. (1991). Multiple regression: Testing and interpreting interactions. Thousand Oaks, CA: Sage.

Allen, T. D., Herst, D. E. L., Bruck, C. S., \& Sutton, M. (2000). Consequences associated with work-to-family conflict: A review and agenda for future research. Journal of Occupational Health Psychology, 5, 278-308. doi: 10.1037//1076-8998.5.2.278

Amstad, F. T., Meier, L. L., Fasel, U., Elfering, A., \& Semmer, N. K. (2011). A metaanalysis of work-family conflict and various outcomes with a special emphasis on cross-domain versus matching domain relations. Journal of Occupational Health Psychology, 16, 151-169. doi: 10.1037/a0022170

Andersson, L. M., \& Pearson, C. M. (1999). Tit for tat? The spiraling effect of incivility in the workplace. The Academy of Management Review, 24, 452-471.

Anger, W. K., Elliot, D. L., Bodner, T., Olson, R., Rohlman, D. S., Truxillo, D. M., Kuehl, K. S., \& Hammer, L. B. (2014). Effectiveness of Total Worker Health interventions. Journal of Occupational Health Psychology. Advance online publication.

Åström, S., Karlsson, S., Sandvide, Å., Bucht, G., Eisemann, M., Norberg, A., \& Saveman, B. I. (2004). Staff's experience of and the management of violent incidents in elderly care. Scandinavian Journal of Caring Sciences, 18, 410-416.

Baer, R. A., Smith, G. T., Hopkins, J., Krietemeyer,J., \& Toney, L. (2006). Using selfreport assessment methods to explore facets of mindfulness. Assessment, 13, 27 45. doi: $10.1177 / 1073191105283504$

Bakker, A. B. \& Demerouti, E. (2007). The job demands-resources model: State of the art. Journal of Managerial Psychology, 22, 309-328. doi:

$10.1108 / 02683940710733115$

Bakker, A. B., Demerouti, E., \& Euwema, M. C. (2005). Job resources buffer the impact of job demands on burnout. Journal of Occupational Health Psychology, 10, 170180. 
Bakker, A. B., Demerouti, E., \& Sanz-Vergel, A. I. (2014). Burnout and work engagement: The JD-R approach. Annual Review of Organizational Psychology and Organizational Behavior, 1, 389-411. doi: 10.1146/annurev-orgpsych031413-091235

Bakker, A. B., Hakanen, J. J., Demerouti, E., \& Xanthopoulou, D. (2007). Job resources boost work engagement, particularly when job demands are high. Journal of Educational psychology, 99, 274. doi: 10.1037/0022-0663.99.2.274

Barbosa, C., Bray, J. W., Brockwood, K., \& Reeves, D. (2014). Costs of a work-family intervention: Evidence from the Work, Family, and Health Network. American Journal of Health Promotion, 28, 209-217.

Barling, J., Dupre, K. E., \& Kelloway, E. K. (2009). Predicting workplace aggression and violence. Annual Review of Psychology, 60, 671-692. doi:

10.1146/annurev.psych.60.110707.163629

Barling, J., Kelloway, E. K., \& Iverson, R. D. (2003). High-quality work, job satisfaction, and occupational injuries. Journal of Applied Psychology, 88, 276-283. doi: 10.1037/0021-9010.88.2.276

Baron, R. M. \& Kenny, D. A. (1986). The moderator-mediator variable distinction in social psychological research: Conceptual, strategic, and statistical considerations. Journal of Personality and Social Psychology, 51, 1173-1182.

Bass, B. L., Butler, A. B., Grzywacz, J. G., \& Linney, K. D. (2009). Do job demands undermine parenting? A daily analysis of spillover and crossover effects. Family Relations: Interdisciplinary Journal of Applied Family Studies, 58, 201-215.

Beck, C., Frank, L., Chumbler, N. R., O'Sullivan, P., Vogelpohl, T. S., Rasin, J., et al., (1998). Correlates of disruptive behavior in severely cognitively impaired nursing home residents. The Gerontologist, 38(2), 189-198.

Beck, C., Rossby, L., \& Baldwin, B. (1991). Correlates of disruptive behavior in cognitively impaired elderly nursing home residents. Archives of Psychiatric Nursing, 5(5), 281-291.

Bigos, S. J., Battie, M. C., Spengler, D. M., Fisher, L. D., Fordyce, W. E., Hansson, T. H., Nachemson, A. L., \& Wortley, M. D. (1991). A prospective study of work perceptions and psychosocial factors affecting the report of back injury. Spine, 16, $1-6$.

Björkqvist, K., Österman, K., \& Hjelt-Bäck, M. (1994). Aggression among university employees. Aggressive Behavior, 20, 173-184.

Bolger, N., DeLongis, A., Kessler, R. C., \& Wethington, E. (1989). The contagion of stress across multiple roles. Journal of Marriage and the Family, 51, 175-183. 
Borman, W. C., \& Motowidlo, S. M. (1993). Expanding the criterion domain to include elements of contextual performance. In N. Schmitt and W. C. Borman (Eds.), Personnel selection in organizations (pp. 71-98). San Francisco: Jossey-Bass.

Bowling, N. A. \& Beehr, T. A. (2006). Workplace harassment from the victim's perspective: A theoretical model and meta-analysis. Journal of Applied Psychology, 91, 998-1012. doi: 10.1037/0021-9010.91.5.998

Bray, J. W., Kelly, E. L., Hammer, L. B., Almeida, D. M., Dearing, J. W., King, R. B., \& Buxton, O. M. (2013). An integrative, multilevel, and transdisciplinary research approach to challenges of work, family, and health. RTI Press publication No. MR-0024-1302. Research Triangle Park, NC: RTI Press. Retrieved from http://www.rti.org/rtipress

Brodsky, C. M. (1976). The harassed worker. Lexington, MA: Lexington Books.

Brown, K. W. \& Ryan, R. M. (2003). The benefits of being present: Mindfulness and its role in psychological well-being. Journal of Personality and Social Psychology, 84, 822-848. doi: 10.1037/0022-3514.84.4.822

Bruk Lee, V. (2006). Measuring social stressors in organizations: The development of the Interpersonal Conflict in Organizations Scale (ICOS). Unpublished doctoral dissertation at the University of South Florida, Tampa, FL.

Bruk-Lee, V. \& Spector, P. E. (2006). The social stressors - counterproductive work behaviors link: Are conflicts with supervisors and coworkers the same? Journal of Occupational Health Psychology, 11, 145-156. doi: 10.1037/1076-8998.11.2.145

Bumpus, M. F., Crouter, A. C., \& McHale, S. M. (1999). Work demands of dual-earner couples: Implications for parents' knowledge about children's daily lives in middle school. Journal of Marriage \& Family, 61, 465-475.

Bureau of Labor Statistics, U. S. Department of Labor. (April 2014). Revisions to the 2012 census of fatal occupational injuries (CFOI) counts. Retrieved from http://www.bls.gov/iif/oshwc/cfoi/cfoi_revised12.pdf

Bureau of Labor Statistics, U. S. Department of Labor. (November 26, 2013). Nonfatal occupational injuries and illness requiring days away from work, 2012. Retrieved from http://www.bls.gov/news.release/pdf/osh2.pdf

Bureau of Labor Statistics, U.S. Department of Labor. (2010). Nonfatal occupational injuries and illnesses requiring days away from work for state government and local government workers, 2008. Retrieved from http://www.bls.gov/news.release/archives/osh2_02242010.pdf

Bushman, B. J., Bonacci, A. M., Pedersen, W. C., Vasquez, E. A., \& Miller, N. (2005). Chewing on it can chew you up: Effects of rumination on triggered displaced 
aggression. Journal of Personality and Social Psychology, 88, 969-983. doi: 10.1037/0022-3514.88.6.969

Butler, C., Scott, J., \& Sussell, A. (2014). Wildland fire fighter activities. Paper presented at the $7^{\text {th }}$ Annual Western States Occupational Network (WestON) Meeting. Golden, Colorado.

Byron, K. (2005). A meta-analytic review of work-family conflict and its antecedents. Journal of Vocational Behavior, 67, 169-198. doi: 10.1016/j.jvb.2004.08.009

Carlson, D., Ferguson, M., Hunter, E., \& Whitten, D. (2012). Abusive supervision and work-family conflict: The path through emotional labor and burnout. The Leadership Quarterly, 23, 849-859. doi:10.1016/j.leaqua.2012.05.003

Casper, L. M., Eby, L. T., Bordeaux, C., Lockwood, A., \& Lambert, D. (2007). A review of research methods in IO/OB work-family research. Journal of Applied Psychology, 92, 28-43. doi: 10.1037/0021-9010.92.1.28

Centers for Disease Control and Prevention. (2015). Total Worker Health ${ }^{\mathrm{TM}}$. Retrieved from http://www.cdc.gov/niosh/twh/

Christian, M. S., Bradley, J. C., Wallace, J. C., \& Burke, M. J. (2009). Workplace safety: A meta-analysis of the roles of person and situation factors. Journal of Applied Psychology, 94, 1103-1127. doi: 10.1037/a0016172

Cho, E. \& Ciancetta, L. (2015). Child outcomes associated with parent work-family experiences. In T. Allen \& L. Eby (Eds.), Oxford Handbook of Work and Family. Oxford University Press.

Clarke, S. (2006). The relationship between safety climate and safety performance: A meta-analytic review. Journal of Occupational Health Psychology, 11, 315-327. doi: 10.1037/1076-8998.11.4.315

Clarke, S. (2012). The effect of challenge and hindrance stressors on safety behavior and safety outcomes: A meta-analysis. Journal of Occupational Health Psychology, 17, 3887-397. doi: 10.1037/a0029817

Clausen, T., Nielsen, K., Carneiro, I. G., \& Borg, V. (2012). Job demands, job resources and long-term sickness absence in the Danish eldercare services: A prospective analysis of register-based outcomes. Journal of Advanced Nursing, 68, 127-136. doi: 10.1111/j.1365-2648.2011.05724.x

Cohen, S., Kamarck, T., \& Mermelstein, R. (1983). A Global Measure of Perceived Stress. Journal of Health and Social Behavior, 24, 385-396.

Cole, M. S., Walter, F., Bedeian, A. G., \& O’Boyle, E. H. (2012). Job burnout and employee engagement: A meta-analytic examination of construct proliferation. Journal of Management, 38, 1550-1581. doi: 10.1177/0149206311415252 
Cortina, L. M., Magley, V. J., Williams, J. H., \& Langhout, R. D. (2001). Incivility in the workplace: Incidence and impact. Journal of Occupational Health Psychology, 6, 64-80. doi: 10.1037//1076-8998.6.1.64

Cropley, M., Michalianou, G., Pravettoni, G., \& Millward, L. J. (2012). The relation of post-work ruminative thinking with eating behavior. Stress and Health, 28, 23-30. doi: $10.1002 /$ smi. 1397

Darling, N. \& Steinberg, L. (1993). Parenting style as context: An integrative model. Psychological Bulletin, 113, 487-496.

Day, A. J., Brasher, K., \& Bridger, R. S. (2012). Accident proneness revisited: The role of psychological stress and cognitive failure. Accident Analysis and Prevention, 49, 532-535. doi: 10.1016/j.aap.2012.03.028

Demerouti, E. \& Bakker, A. B. (2011). The job demands-resources model: Challenges for future research. South African Journal of Industrial Psychology, 37, 1-9. doi: 10.4102/sajip.v37i2.974

Demerouti, E. \& Bakker, A. B. (2011). The job demands-resources model: Challenges for future research. South African Journal of Industrial Psychology, 37, 1-9. doi: 10.4102/sajip.v37i2.974

Demerouti, E., Bakker, A. B., \& Bulters, A. (2004). The loss spiral of work pressure, work-home interface and exhaustion: Reciprocal relations in a three-wave study. Journal of Vocational Behavior, 4, 131-149.

Demerouti, E., Bakker, A. B., Geurts, S. A. E., \& Taris, T. W. (2009). Daily recovery from work-related effort during non-work time. Current Perspectives on JobStress Recovery, 7, 85-123. doi: 10.1108/S1479-3555(2009)0000007006

Demerouti, E., Bakker, A. B., Nachreiner, F., \& Schaufeli, W. B. (2001). The job demands-resources model of burnout. Journal of Applied Psychology, 86, 499512. doi: 10.1037//0021-9010.86.3.499

Demsky, C. A. \& Fritz, C. (in progress). Workplace aggression and occupational safety: A moderated mediation model.

Demsky, C. A., \& Hammer, L. B. (in progress). The Role of Perceived Stress in the Relationship between Workplace Aggression and Parenting: Evidence from the Work, Family, \& Health Network.

Demsky, C. A., Ellis, A. M., \& Fritz, C. (2014). Shrugging it off: Does psychological detachment mediate the relationship between workplace aggression and workfamily conflict? Journal of Occupational Health Psychology, 19, 195-205. doi: $10.1037 / \mathrm{a} 0035448$ 
Dierdorff, E. C., \& Ellington, J. K. (2008). It's the nature of the work: Examining behavior-based sources of work-family conflict across occupations. Journal of Applied Psychology, 93, 883-892.

Donahue, E. G., Forest, J., Vallerand, R. J., Lemyre, P.-N., Crevier-Braud, L., \& Bergeron, E. (2012). Passion for work and emotional exhaustion: The mediating role of rumination and recovery. Applied Psychology: Health and Well-Being, 4, 341-368. doi: 10.1111/j.1758-0854.2012.01078.x

Douglas, S. C., Kiewitz, C., Martinko, M. J., Harvey, P., Kim, Y., \& Chun, J. U. (2008). Cognitions, emotions, and evaluations: An elaboration likelihood model for workplace aggression. Academy of Management Review, 33, 425-451.

Duffy, M. K., Ganster, D. C., \& Pagon, M. (2002). Social undermining in the workplace. Academy of Management Journal, 45, 331-351. doi: 10.2307/3069350

Duffy, M., \& Sperry, L. (2007). Workplace mobbing: Individual and family health consequences. The Family Journal: Counseling and Therapy for Couples and Families, 15, 398-404. doi: 10.1177/1066480707305069

Dupre, K.E. \& Barling, J. (2006). Predicting and preventing supervisory workplace aggression. Journal of Occupational Health Psychology, 11, 13-26. doi: 10.1037/1076-8998.11.1.13

Duxbury, L. E., \& Higgins, C. A. (1991). Gender differences in work-family conflict. Journal of Applied Psychology, 76, 60-74. doi: 10.1037/0021-9010.76.1.60

Eberth, J. \& Sedlmeier, P. (2012). The effects of mindfulness meditation: A metaanalysis. Mindfulness, 3, 174-189.

Edward, K. L., Ousey, K., Warelow, P., \& Lui, S. (2014). Nursing and aggression in the workplace: a systematic review. British Journal of Nursing, 23, 653-659.

Edwards. J. R., \& Rothbard, N. P. (2000). Mechanisms linking work and family: Clarifying the relationship between work and family constructs. The Academy of Management Review, 25, 178-199. doi: 10.5465/AMR.2000.2791609

Einarsen, S. (1999). The nature and causes of bullying at work. International Journal of Manpower, 20, 16-27. doi: 10.1108/01437729910268588

Einarsen, S. (2000). Harassment and bullying at work: A review of the Scandinavian approach. Aggression and Violent Behavior: A Review Journal, 5, 371-401.

Eisinga, R., te Grotenhuis, M., \& Pelzer, B. (2013). The reliability of a two-item scale: Pearson, Cronbach, or Spearman-Brown? International Journal of Public Health, 58, 637-642. doi: 10.1007/s00038-012-0416-3 
Elfering, A., Grebner, S., \& Dudan, A. (2011). Job characteristics in nursing and cognitive failure at work. Safety and Health at Work, 2, 194-200. doi: 10.5491/SHAW.2011.2.2.194

Etzion, D., Eden, D., \& Lapidot, Y. (1998). Relief from job stressors and burnout: Reserve service as a respite. Journal of Applied Psychology, 83, 577-585. doi: 10.1037/0021-9010.83.4.577

Farrell, L. U. (2002, March 15). Workplace bullying's high cost: $\$ 180 \mathrm{M}$ in lost time, productivity. Orlando Business Journal. Retrieved July 24, 2014 from: http://www.careerbuilder.com/share/aboutus/pressreleasesdetail.aspx?id=pr632\&s $\mathrm{d}=4 \% 2 \mathrm{~F} 20 \% 2 \mathrm{~F} 2011 \& \mathrm{~d}=4 \% 2 \mathrm{~F} 20 \% 2 \mathrm{~F} 2099$

Feldman, G., Hayes, A., Kumar, S., Greeson, J., \& Laurenceau, J.-P. (2007). Mindfulness and emotion regulation: The development and initial validation of the cognitive and affective mindfulness scale-revised (CAMS-R). Journal of Psychopathology and Behavioral Assessment, 29, 177-190. doi: 10.1007/s10862-006-9035-8

Feltman, R., Robinson, M. D., \& Ode, S. (2009). Mindfulness as a moderator of neuroticism-outcome relations: A self-regulation perspective. Journal of Research in Personality, 43, 953-961. doi: 10.1016/j.jrp.2009.08.009

Ford, M. T., Heinen, B. A., \& Langkamer, K. L., (2007). Work and family satisfaction and conflict: A meta-analysis of cross-domain relations. Journal of Applied Psychology, 92, 57-80. doi: 10.1037/0021-9010.92.1.57

Fox, S., Spector, P. E., \& Miles, D. (2001). Counterproductive work behavior (CWB) in response to job stressors and organizational justice: Some mediator and moderator tests for autonomy and emotions. Journal of Vocational Behavior, 59, 291-309. doi: $10.1006 /$ jvbe. 2001.1803

Fritz, C., \& Sonnentag, S. (2005). Recovery, health, and job performance: Effects of weekend experiences. Journal of Occupational Health Psychology, 10, 187-199. doi: 10.1037/1076-8998.10.3.187

Fritz, C., Ellis, A. M., Demsky, C. A., Lin, B. C., \& Guros, F. (2013). Embracing work breaks: Recovering from work stress. Organizational Dynamics, 42, 274-280.

Fritz, C., Yankelevich, M., Zarubin, A., \& Barger, P. (2010). Happy, healthy, and productive: The role of detachment from work during nonwork time. Journal of Applied Psychology, 95, 977-983. doi: 10.1037/a0019462

Frone, M. R. (1999). Work stress and alcohol use. Alcohol Research and Health, 23, 284291. 
Frone, M. R., Russell, M., \& Cooper, M. L. (1992). Prevalence of work-family conflict: Are work and family boundaries asymmetrically permeable? Journal of Organizational Behavior, 13, 723-729. doi: 10.1002/job.4030130708

Fujishiro, K., Gee, G. C., \& de Castro, A. B. (2011). Associations of workplace aggression with work-related well-being among nurses in the Phillipines. American Journal of Public Health, 101, 861-867. doi: 10.2105/AJPH.2009.188144

Garcia, P. R. J. M., Restubog, S. L. D., Kiewitz, C., Scott, K. L., \& Tang, R. L. (2014). Roots run deep: Investigating psychological mechanisms between history of family aggression and abusive supervision. Journal of Applied Psychology, 99, 883-897. doi: 10.1037/a0036463

Gassman-Pines, A. (2011). Associations of low-income working mothers' daily interactions with supervisors and mother-child interactions. Journal of Marriage and Family, 73, 67-76.

Gates, D. M., Fitzwater, E., \& Meyer, U. (1999). Violence against caregivers in nursing homes. Expected, tolerated, and accepted. Journal of Gerontological Nursing, 25(4), 12-22.

Gerberich, S. G., Church, T. R., Church, McGovern, P. M., Hansen, H. E., Nachreiner, N. M., Geisser, M. S., Ryan, A. D., Mongin, S. J., \& Watt, G. D. (2004). An epidemiological study of the magnitude and consequences of work related violence: The Minnesota nurses' study. Occupational and Environmental Medicine, 61, 495-503. doi: 10.1136/oem.2003.007294

Geriatric Mental Health Foundation (2014). Alzheimer's and related dementias (fact sheet). Retrived October 13, 2014, from http://www.gmhfonline.org/gmhf/consumer/factsheets/dementia_factsheet.html.

Glomb, T. M., Duffy, M. K., Bono, J. E., \& Yang, T. (2011). Mindfulness at work. Research in Personnel and Human Resources Management, 30, 115-157. doi: 10.1108/S0742-7301(2011)0000030005

Goldenhar, L. M., Williams, L. J., \& Swanson, N. G. (2003). Modeling relationships between job stressors and injury and near-miss outcomes for construction labourers. Work \& Stress, 17, 218-240.

Green, S. G., Schaefer, R. A. B., MacDermid, S. M., \& Weiss, H. M. (2011). Partner reactions to work-to-family conflict: Cognitive appraisal and indirect crossover in couples. Journal of Management, 37, 744-769. doi: 10.1177/0149206309349307 
Greenberger, E., O’Neil, \& Nagel, S. K. (1994). Linking workplace and homeplace: Relations between the nature of adults' work and their parenting behaviors. Developmental Psychology, 30, 990-1002.

Greenhaus, J. H. \& Beutell, N. J. (1985). Sources of conflict between work and family roles. Academy of Management Review, 10, 76-88. doi: 10.5465/AMR.1985.4277352

Grossman, P., Niemann, L., Schmidt, S., \& Walach, H. (2004). Mindfulness-based stress reduction and health benefits: A meta-analysis. Journal of Psychosomatic Research, 57, 35-43. doi: 10.1016/S0022-3999(03)00573-7

Grzywacz, J. G., \& Marks, N. F. (2000). Reconceptualizing the work-family interface: An ecological perspective on the correlates of positive and negative spillover between work and family. Journal of Occupational Health Psychology, 5, 111. doi: 10.1037111076-8998.5.1.111

Hahn, V. C., Binnewies, C., Sonnentag, S., \& Mojza, E. J. (2011). Learning how to recover from job stress: Effects of a recovery training program on recovery, recovery-related self-efficacy, and well-being. Journal of Occupational Health Psychology, 16, 202-216. doi: 10.1037/a0022169

Halbesleben, J. R. B., Neveu, J.-P., Paustian-Underdahl, S. C., \& Westman, M. (2014). Getting to the "COR": Understanding the role of resources in Conservation of Resources theory. Journal of Management, 40, 1334-1364.

Hammer, L. B. \& Sauter, S. (2013). Total worker health and work-life stress. Journal of Occupational and Environmental Medicine, 55, S25 - S29.

Hammer, L. B., Demsky, C.A., Kossek, E., \& Bray, J. (2015). Intervention research. In T. Allen \& L. Eby (Eds.), Oxford Handbook of Work and Family. Oxford University Press.

Hammer, L.B., \& Zimmerman, K. L. (2011). Quality of work life. In S. Zedeck (Ed.), APA Handbook of Industrial and Organizational Psychology, Vol. 3, (pp. 399431). Washington, DC: American Psychological Association.

Hanson, G. C., \& Hammer, L. B., \& Colton, C. L. (2006). Development and validation of a multidimensional scale of perceived work-family positive spillover. Journal of Occupational Health Psychology, 11, 249-265.

Hanson, G. C., Perrin, N. A., Moss, H., Laharnar, N., \& Glass, N. (2015). Workplace violence against homecare workers and its relationship with workers' health outcomes: A cross-sectional study. BMC Public Health, 15. 
Hayes, A. F., \& Preacher, K., J. (2014). Statistical mediation analysis with a multicategorical independent variable. British Journal of Mathematical and Statistical Psychology, 67, 451-470.

Hayes, B. E., Perander, J., Smecko, T., \& Trask, J. (1998). Measuring perceptions of workplace safety: Development and validation of the work safety scale. Journal of Safety Research, 29, 145-161.

Hemingway, M. A. \& Smith, C. S. (1999). Organizational climate and occupational stressors as predictors of withdrawal behaviours and injuries in nurses. Journal of Occupational and Organizational Psychology, 72, 285-299.

Herndon, F. (2008). Testing mindfulness with perceptual and cognitive factors: External vs. internal encoding, and the cognitive failures questionnaire. Personality and Individual Differences, 44, 32-41. doi: 10.1016/j.paid.2007.07.002

Hershcovis, M. S. (2011). "Incivility, social undermining, bullying...oh my!": A call to reconcile constructs within workplace aggression research. Journal of Organizational Behavior, 32, 499-519. doi: 10.1002/job.689

Herschovis, M. S., \& Barling, J. (2006). Preventing insider-initiated violence. In K. Kelloway, J. Barling, J.J. Hurrell (Eds.). Handbook of Workplace Violence, (pp. 607-31). Thousand Oaks, CA: Sage.

Hershcovis, M. S. \& Barling, J. (2010). Towards a multi-foci approach to workplace aggression: A meta-analytic review of outcomes from different perpetrators. Journal of Organizational Behavior, 31, 24-44. doi: 10.1002/job.621

Hershcovis, M. S., Turner, N., Barling, J., Arnold, K. A., Dupre, K. E., Inness, M., LeBlanc, M. M., \& Sivanathan, N. (2007). Predicting workplace aggression: A meta-analysis. Journal of Applied Psychology, 92, 228-238. doi: 10.1037/00219010.92.1.228

Hobfoll, S. E. (1989). Conservation of resources: A new attempt at conceptualizing stress. American Psychology, 44, 513-524.

Hobfoll, S. E. (1998). Stress, culture, and community. New York: Plenum.

Hobfoll, S. E. (2001). The influence of culture, community, and the nested self in the stress process: Advancing conservation of resources theory. Applied Psychology: An International Review, 50, 337-370.

Hobfoll, S. E. (2002). Social and psychological resources and adaptation. Review of General Psychology, 6, 307-324. doi:10.1037/1089-2680.6.4.307 
Hobfoll, S. E., Johnson, R. J., Ennis, N., Jackson, A. P. (2003). Resource loss, resource gain, and emotional outcomes among inner city women. Journal of Personality and Social Psychology, 84, 632-643. doi: 10.1037/0022-3514.84.3.632

Hobfoll, S.E., \& Shirom, A. (2000). Conservation of resources theory: Application to stress and management in the workplace. In R.T. Golembiewski (Ed.), Handbook of organizational behavior (pp. 57-81). New York, NY: Dekker.

Hofmann, D. A., Morgeson, F. P., \& Gerras, S. J. (2003). Climate as a moderator of the relationship between leader-member exchange and content specific citizenship: Safety climate as an exemplar. Journal of Applied Psychology, 88, 170-178. doi: 10.1037/0021-9010.88.1.170

Hoobler, J. M., \& Brass, D. J. (2006). Abusive supervision and family undermining as displaced aggression. Journal of Applied Psychology, 91, 1125-1133. doi: 10.1037/0021-9010.91.5.1125

Hulsheger, U. R., Alberts, H. J. E. M., Feinholdt, A., \& Lang, J. W. B. (2013). Benefits of mindfulness at work: The role of mindfulness in emotion regulation, emotional exhaustion, and job satisfaction. Journal of Applied Psychology, 98, 310-325. doi: $10.1037 / \mathrm{a} 0031313$

International Labour Organization. (n.d.). Safety and health at work. Retrieved from http://ilo.org/global/topics/safety-and-health-at-work/lang--en/index.htm

Islam, S. S., Edla, S. R., Mujuru, P., Doyle, E. J., \& Ducatman, A. M. (2003). Risk factors for physical assault: state-managed workers' compensation experience. American Journal of Preventive Medicine, 25(1), 31-37.

Jiang, L., Yu, G., Li, Y., \& Li, F. (2010). Perceived colleagues' safety knowledge/behavior and safety performance: Safety climate as a moderator in a multilevel study. Accident Analysis and Prevention, 42, 1468-1476. doi: 10.1016/j.aap.2009.08.017

Judge, T. A. \& Bono, J. E. (2001). Relationship of core self-evaluations traits - selfesteem, generalized self-efficacy, locus of control, and emotional stability - with job satisfaction and job performance: A meta-analysis. Journal of Applied Psychology, 86, 80-92.

Judge, T. A., van Vianen, A. E. M., \& de Pater, I. E. (2004). Emotional stability, core self-evaluations, and job outcomes: A review of the evidence and an agenda for future research. Human Performance, 17, 325-346.

Kabat-Zinn, J. (1990). Full catastrophe living. New York: Delta.

Kabat-Zinn, J. (1994). Wherever you go, there you are: Mindfulness meditation in everyday life. New York: Hyperion. 
Kahn, R. L., Wolfe, D. M., Quinn, R., Snoek, J. D., \& Rosenthal, R. A. (1964).

Organizational stress. New York: Wiley.

Katz, D., \& Kahn, R. L. (1978). The social psychology of organizations. New York: Wiley.

Keenan, A., \& Newton, T. J. (1985). Stressful events, stressors, and psychological strains in young professional engineers. Journal of Occupational Behavior, 6, 151-156. doi: $10.1002 /$ job.4030060206

Kenny, D. A., Kashy, D. A., \& Bolger, N. (1998). Data analysis in social psychology. The handbook of social psychology, 1, 233-265.

Kingston, T., Dooley, B., Bates, A., Lawlor, E., \& Malone, K. (2007). Mindfulness-based cognitive therapy for residual depressive symptoms. Psychology and Psychotherapy: Theory, Research, and Practice, 80, 193-203. doi: 10.1348/147608306X116016

Kossek, E. E., Hammer, L. B., Kelly, E. L., \& Moen, P. (2014). Designing work, family, $\&$ health organizational change initiatives. Organizational Dynamics, 43, 53-63. doi: 10.1016/j.orgdyn.2013.10.007

Kossek, E., Lewis, S., \& Hammer, L. (2010). Work-life initiatives and organizational change: Overcoming mixed messages to move from the margin to the mainstream. Human Relations, 63, 3-19. doi: 10.1177/0018726709352385

Larson, G. E., Alderton, D. L., Neideffer, M., \& Underhill, E. (1997). Further evidence on dimensionality and correlates of the cognitive failures questionnaire. British Journal of Psychology, 88, 29-38.

Larson, G. E. \& Merrit, C. R. (1991). Can accidents be predicted? An empirical test of the cognitive failures questionnaire. Applied Psychology: An International Review, 40, 37-45.

Lazarus, R. S. (1966). Psychological stress and the coping process. New York: McGrawHill.

Leigh, J. P. (2011). Economic burden of occupational injury and illness in the United States. The Milbank Quarterly: A Multidisciplinary Journal of Population Health and Health Policy, 89, 728-772.

Leiter, M. P., Laschinger, H. K. S., Day, A., \& Oore, D. G. (2011). The impact of civility interventions on employee social behavior, distress, and attitudes. Journal of Applied Psychology, 96, 1258-1274. doi: 10.1037/a0024442

Level Playing Field Institute. (2007). Corporate Leavers Survey. Retrieved July 24, 2014 from: http://www.lpfi.org/workplace/corporateleavers.html 
Lewis, S. E., \& Orford, J. (2005). Women's experience of workplace bullying: Changes in social relationships. Journal of Community \& Applied Social Psychology, 15, 29-47. doi: 10.1002/casp.807

Leymann, H. (1990). Mobbing and psychological terror at workplaces. Violence and Victims, 5, 119-126.

Lim, S., \& Cortina, L. M. (2005). Interpersonal mistreatment in the workplace: the interface and impact of general incivility and sexual harassment. Journal of Applied Psychology, 90, 483.

Lim, S., \& Lee, A. (2011). Work and nonwork outcomes of workplace incivility: Does family support help? Journal of Occupational Health Psychology, 16, 95-111. doi: $10.1037 / \mathrm{a} 0021726$

Lim, S., Cortina, L. M., \& Magley, V. J. (2008). Personal and workgroup incivility: Impact on work and health outcomes. Journal of Applied Psychology, 93, 95-107. doi: 10.1037/0021-9010.93.1.95

Linehan, M. M., Comtois, K. A., Murray, A. M., Brown, M. Z., Gallop, R. J., Heard, H. L., Korslund, K. E., Tutek, D. A., Reynolds, S., \& Lindenboim, N. (2006). Twoyear randomized controlled trial and follow-up of dialectical behavior therapy vs. therapy by experts for suicidal behaviors and borderline personality disorder. Archives of General Psychiatry, 63, 757-766.

Liu, Y, Wang, M., Chang, C.-H., Shi, J., Zhou, L., \& Shao, R. (2014, December 22). Work-family conflict, emotional exhaustion, and displaced aggression toward others: The moderating roles of workplace interpersonal conflict and perceived managerial family support. Journal of Applied Psychology. Advance online publication. doi: 10.1037/a0038387

MacEwen, K. E. \& Barling, J. (1991). Effects of maternal employment experiences on children's behavior via mood, cognitive difficulties, and parenting behavior. Journal of Marriage and Family, 53, 635-644.

MacKinnon, D. P., Lockwood, C. M., \& Williams, J. (2004). Confidence limits for the indirect effect: Distribution of the product and resampling methods. Multivariate Behavioral Research, 39, 99-128. doi: 10.1207/s15327906mbr3901_4

Mandler, G. (1979). Thought processes, consciousness, and stress. In V. Hamilton \& D.M. Warburton (eds.), Human stress and cognition: An information processing approach. London: Wiley.

Martin, M. (1983). Cognitive failure: Everyday and laboratory performance. Bulletin of Psychonomic Society, 21, 97-100. 
McKee, L, Roland, E., Coffelt, N., Olson, A. L., Forehand, R., Massari, C., Jones, D., Gaffney, C. A., \& Zens, M. S. (2007). Harsh discipline and child problem behaviors: The roles of positive parenting and gender. Journal of Family Violence, 22, 187-196.

Meijman, T. F., \& Mulder, G. (1998). Psychological aspects of workload. In P. J. D. Drenth \& H. Thierry (Eds.), Handbook of work and organizational psychology (Vol. 2: Work psychology, pp. 5-33). Hove, England: Psychology Press.

Michel, J. S., Kotrba, L. M., Mitchelson, J. K., Clark, M. A., \& Baltes, B. B. (2011). Antecedents of work-family conflict: A meta-analytic review. Journal of Organizational Behavior, 32, 689-725. doi: 10.1002/job.695

Miranda, H., Punnett, L., \& Gore, R. J. (2014). Musculoskeletal Pain and Reported Workplace Assault A Prospective Study of Clinical Staff in Nursing Homes. Human Factors: The Journal of the Human Factors and Ergonomics Society, 56, 215-227.

Miranda, H., Punnett, L., Gore, R., \& Boyer, J. (2010). Violence at the workplace increases the risk of musculoskeletal pain among nursing home workers. Occupational and Environmental Medicine.

Moreno-Jimenez, B., Mayo, M., Sanz-Vergel, A. I., Geurts, S., Rodriguez-Munoz, A., \& Garrosa, E. (2009). Effects of work-family conflict on employees' well-being: The moderating role of recovery strategies. Journal of Occupational Health Psychology, 14, 427-440. doi: 10.1037/a0016739

Moreno-Jimenez, B., Rodriquez-Munoz, A., Pastor, J. C., Sanz-Vergel, A. I., \& Garrosa, E. (2009). The moderating effects of psychological detachment and thoughts of revenge in workplace bullying. Personality and Individual Differences, 46, 359364. doi: 10.1016/j.paid.2008.10.031

Morgan D.G., Stewart N.J., D’Arcy C., Forbes D. \& Lawson J. (2005). Work stress and physical assault of nursing aides in rural nursing homes with and without dementia special care units. Journal of Psychiatric and Mental Health Nursing, $12,347-358$.

Muller, D., Judd, C. M., \& Yzerbyt, V. Y. (2005). When moderation is mediated and mediation is moderated. Journal of Personality and Social Psychology, 89, 852863. doi: 10.1037/0022-3514.89.6.852

Muthén, L. K. \& Muthén, B. O. (1998 - 2010). Mplus User's Guide. Sixth Edition. Los Angeles, CA: Muthén \& Muthén. 
Nahrgang, J. D., Morgeson, F. P., \& Hofman, D. A. (2011). Safety at work: A metaanalytic investigation of the link between job demands, job resources, burnout, engagement, and safety outcomes. Journal of Applied Psychology, 96, 71-94.

Narayanan, L., Menon, S., \& Spector, P. (1999). A cross-cultural comparison of job stressors and reactions among employees holding comparable jobs in two countries. International Journal of Stress Management, 6, 197-212. doi: 10.1023/A:1021986709317

National Institute for Occupational Safety and Health. (2010). Occupational health psychology (OHP). Retrieved from http://www.cdc.gov/niosh/topics/ohp/

Neal, A. \& Griffin, M. A. (2006). A study of the lagged relationships among safety climate, safety motivation, safety behavior, and accidents at the individual and group levels. Journal of Applied Psychology, 91, 946-953. doi: 10.1037/00219010.91.4.946

Neal, A., Griffin, M. A., \& Hart, P. M. (2000). The impact of organizational climate on safety climate and individual behavior. Safety Science, 34, 99-109.

Netemeyer, R. G., Boles, J. S., \& McMurrian, R. (1996). Development and validation of work-family conflict and family-work conflict scales. Journal of Applied Psychology, 81, 400-410. doi: 10.1037/0021-9010.81.4.400

Neuman, J. H., \& Baron, R. A. (1997). Aggression in the workplace. In R. Giacalone and J. Greenberg (Eds.), Antisocial behavior in organizations (pp. 37-67). Thousand Oaks, CA: Sage.

Niven, K., Sprigg, C. A., Armitage, C. J., \& Satchwell, A. (2013). Ruminative thinking exacerbates the negative effects of workplace violence. Journal of Occupational and Organizational Psychology, 86, 67-84. doi: 10.1111/j.20448325.2012.02066.x

Nixon, A. E. (2011). Charting a semantic jungle: A novel method for examining the moderators of workplace aggression. Unpublished doctoral dissertation at the University of South Florida, Tampa, FL.

Nixon, A. E., Mazzola, J. J., Bauer, J., Krueger, J. R., \& Spector, P. E. (2011). Can work make you sick? A meta-analysis of the relationships between job stressors and physical symptoms. Work \& Stress: An International Journal of Work, Health, \& Organizations, 25, 1-22. doi:10.1080/02678373.2011.569175

Nixon, R. (2014, September 19). Women allege harassment and abuse on Forest Service firefighting crews. The New York Times. Retrieved from http://www.nytimes.com/2014/09/20/us/Women-Allege-Harassment-and-Abuseon-Forest-Service-Firefighting-Crews.html?emc=eta1 
Occupational Safety and Health Administration. (2004). Guidelines for preventing workplace violence for health care social service workers. In Guidelines for preventing workplace violence for health care social service workers. OSHA.

Occupational Safety and Health Administration. (2015). Safety and health topics: Workplace Violence. Retrieved from: https://www.osha.gov/SLTC/workplaceviolence/

Okechukwu, C. A., Souza, K., Davis, K. D., \& de Castro, A. B. (2014). Discrimination, harassment, abuse, and bullying in the workplace: Contributions of workplace injustice to occupational health disparities. American Journal of Industrial Medicine, 57, 573-586. doi: 10.1002/ajim.

Osatuke, K., Leiter, M., Belton, L., Dyrenforth, S., \& Ramsel, D. (2013). Civility, Respect, and Engagement at the Workplace (CREW): A national organization development program at the Department of Veterans Affairs. Journal of Management Policies and Practices, 1, 25-34.

Osatuke, K., Moore, S. C., Ward, C., Dyrenforth, S. R., \& Belton, L. (2009). Civility, Respect, Engagement in the Workforce (CREW). The Journal of Applied Behavioral Science, 45, 384-410.

Park, Y., Fritz, C., \& Jex, S. M. (2011). Relationships between work-home segmentation and psychological detachment from work: The role of communication technology use at home. Journal of Occupational Health Psychology, 16, 457-467. doi: $10.1037 / \mathrm{a} 0023594$

Pearson, C. M. \& Porath, C. L. (2005). On the nature, consequences and remedies of workplace incivility: No time for "nice"? Think again. Academy of Management Executive, 19, 7-18. doi: 10.5465/AME.2005.15841946

Penney, L. M. \& Spector, P. E. (2005). Job stress, incivility, and counterproductive work behavior (CWB): The moderating role of negative affectivity. Journal of Organizational Behavior, 26, 777-796. doi: 10.1002/job.336

Pinquart, M. \& Sörenson, S. (2003). Differences between caregivers and noncaregivers in psychological health and physical health: A meta-analysis. Psychology and Aging, 18, 250-267. doi: 10.1037/0882-7974.18.2.250

Podsakoff, N. P., LePine, J. A., \& LePine, M. A. (2007). Differential challenge stressorhindrance stressor relationships with job attitudes, turnover intentions, turnover, and withdrawal behavior: A meta-analysis. Journal of Applied Psychology, 92, 438-454. doi: 10.1037/0021-9010 .92.2.438

Podsakoff, P. M., MacKenzie, S. B., Lee, J.-Y., \& Podsakoff, N. P. (2003). Common method biases in behavioral research: A critical review of the literature and 
recommended remedies. Journal of Applied Psychology, 88, 879-903. doi: 10.1037/0021-9010.88.5.879

Porath, C., \& Pearson, C. (2010). The cost of bad behavior. Organizational Dynamics, 39, 4-71. doi:10.1016/j.orgdyn.2009.10.006

Preacher, K. J., \& Hayes, A. F. (2004). SPSS and SAS procedures for estimating indirect effects in simple mediation models. Behavior Research Methods, Instruments, and Computers, 36, 717-731. doi: 10.3758/BF03206553

Preacher, K. J., \& Hayes, A. F. (2008). Asymptotic and resampling strategies for assessing and comparing indirect effects in multiple mediator models. Behavior Research Methods, 40, 879-891. doi:10.3758/BRM.40.3.879

Preacher, K. J., Rucker, D. D., \& Hayes, A. F. (2007). Addressing moderated mediation hypotheses: Theory, methods, and prescriptions. Multivariate Behavioral Research, 42, 185-227.

Querstret, D. \& Cropley, M. (2012). Exploring the relationship between work-related rumination, sleep quality, and work-related fatigue. Journal of Occupational Health Psychology, 17, 341-353.

Quick, J. C. (1999). Occupational health psychology: The convergence of health and clinical psychology with public health and preventative medicine in an organizational context. Professional Psychology: Research and Practice, 30, 123128.

Quine, L. (1999). Workplace bullying in NHS community trust: Staff questionnaire survey. British Medical Journal, 318, 228-232. doi: 10.1136/bmj.318.7178.228

Raes, F. \& Williams, M. G. (2010). The relationship between mindfulness and uncontrollability of ruminative thinking. Mindfulness, 1, 199-203. doi: $10.1007 / \mathrm{s} 12671-010-0021-6$

Ransford, C. R., Crouter, A. C., \& McHale, S. M. (2008). Implications of work pressure and supervisor support for fathers', mothers' and adolescents' relationships and well-being in dual-earner families. Community, Work, \& Family, 11, 37-60.

Repetti, R. L. (1989). Effects of daily workload on subsequent behavior during marital interaction: The roles of social withdrawal and spouse support. Journal of Personality and Social Psychology, 57, 651-659. doi: 10.1037/00223514.57.4.651

Repetti, R. L. \& Wood, J. (1997). Effects of daily stress at work on mothers' interactions with preschoolers. Journal of Family Psychology, 11, 90-108. 
Restubog, S. L. D., Scott, K. L., \& Zagenczyk, T. J. (2011). When distress hits home: The role of contextual factors and psychological distress in predicting employees' responses to abusive supervision. Journal of Applied Psychology, 96, 713-729. doi: 10.1037/A0021593

Rocky Mountain Research Station, Human Factors \& Risk Management. (n.d.). Projects. Retrieved from http://www.fs.fed.us/rm/human-factors/projects/

Roeters, A., van der Lippe, T., \& Kluwer, E. S. (2010). Work characteristics and parentchild relationship quality: The mediating role of temporal involvement. Journal of Marriage and Family, 72, 1317-1328.

Ryden, M. B., Bossenmaier, M., \& McLachlan, C. (1991). Agressive behavior in cognitively impaired nursing home residents. Research in Nursing \& Health, 14(2), 87-95.

Sanz-Vergel, A. I., Demerouti, E., Bakker, A. B., \& Moreno-Jimenez, B. (2011). Daily detachment from work and home: The moderating effect of role salience. Human Relations, 64, 775-799. doi: 10.1177/0018726710393368

Saxbe, D. E., Repetti, R. L., \& Graesch, A. P. (2011). Time spent in housework and leisure: Links with parents' physiological recovery from work. Journal of family psychology, 25, 271-281. doi: 10.1037/a0023048

Schaefer, E.S. (1965). Children's reports of parental behavior: An inventory. Child Development, 36, 417-424.

Schat, A. C. H., Frone, M. R., \& Kelloway, E. K. (2006). Prevalence of workplace aggression in the U. S. workforce. In E. K. Kelloway, J. Barling, \& J. J. Hurrell (Eds.). Handbook of workplace violence. Thousand Oaks, CA: Sage Publications.

Schat, A. C., \& Kelloway, E. K. (2000). Effects of perceived control on the outcomes of workplace aggression and violence. Journal of Occupational Health Psychology, 5,386 .

Schat, A. C., \& Kelloway, E. K. (2003). Reducing the adverse consequences of workplace aggression and violence: the buffering effects of organizational support. Journal of occupational health psychology, 8, 110. doi: 10.1037/10768998.8.2.110

Schaufeli, W. B. \& Bakker, A. B. (2004). Job demands, job resources, and their relationship with burnout and engagement: A multi-sample study. Journal of Organizational Behavior, 25, 293-315. doi: 10.1002/job.248 
Schwarz, J.C., Barton-Henry, M. L., \& Pruzinsky, T. (1985). Assessing child-rearing behavior: A comparison of ratings made by mother, father, child, and sibling on the CRPBI. Child Development, 56, 462-479.

Segal, Z. V., Williams, J. M. G., \& Teasdale, J. D. (2002). Mindfulness based cognitive therapy for depression: A new approach to preventing relapse. New York: Guilford Press.

Selig, J. P. \& Preacher, K. J. (2009). Mediation models for longitudinal data in developmental research. Research in Human Development, 6, 144-164. doi: $10.1080 / 15427600902911247$

Setti, I. \& Argentero, P. (2014). The role of mindfulness in protecting firefighters from psychosomatic malaise. Traumatology: An International Journal, 20, 134-141. doi: $10.1037 / \mathrm{h} 0099398$

Shapiro, S. L., Brown, K. W., Thoreson, C., \& Plante, T. G. (2011). The moderation of mindfulness-based stress reduction effects by trait mindfulness: Results from a randomized controlled trial. Journal of Clinical Psychology, 67, 267-277. doi: 10.1002/jclp.20761

Shrout, P. E., \& Bolger, N. (2002). Mediation in experimental and nonexperimental studies: New procedures and recommendations. Psychological Methods, 7, 422445. doi: 10.1037/1082-989X.7.4.422

Siu, O. L., Cooper, C. L., \& Phillips, D. R. (2014). Intervention studies on enhancing work well-being, reducing burnout, and improving recovery experiences among Hong Kong health care workers and teachers. International Journal of Stress Management, 21, 69-84.

Snyder, L. A., Chen, P. Y., \& Vacha-Haase, T. (2007). The underreporting gap in aggressive incidents from geriatric patients against certified nursing assistants. Violence and Victims, 22(3), 367-379.

Sonnentag, S. (2012). Psychological detachment from work during leisure time: The benefits of mentally disengaging from work. Current Directions in Psychological Science, 21, 114-118. doi: 10.1177/0963721411434979

Sonnentag, S., \& Bayer, U.-V. (2005). Switching off mentally: Predictors and consequences of psychological detachment from work during off-job time. Journal of Occupational Health Psychology, 10, 393-414. doi: 10.1037/10768998.10.4.393

Sonnentag, S., Binnewies, C., \& Mojza, E. J. (2008). Did you have a nice evening? A day-level study on recovery experiences, sleep, and work-relevant affect. Journal of Applied Psychology, 93, 674-684. doi: 10.1037/0021-9010.93.3.674 
Sonnentag, S., Binnewies, C., \& Mojza, E. J. (2010). Staying well and engaged when demands are high: The role of psychological detachment. Journal of Applied Psychology, 95, 965-976. doi: 10.1037/a002003

Sonnentag, S., \& Fritz, C. (2007). The Recovery Experience Questionnaire: Development and validation of a measure for assessing recuperation and unwinding from work. Journal of Occupational Health Psychology, 12, 204-221. doi:10.1037/10768998.12.3.204

Sonnentag, S., Kuttler, I., \& Fritz, C. (2010). Job stressors, emotional exhaustion, and need for recovery: A multi-source study on the benefits of psychological detachment. Journal of Vocational Behavior, 76, 355-365. doi:10.1016/j.jvb.2009.06.005

Sonnentag, S., \& Niessen, C. (2008). Staying vigorous until work is over: The role of trait vigour, day-specific work experiences and recovery. Journal of Occupational and Organizational Psychology, 81, 435-458. doi: 10.1348/096317908X310256

Spector, P. E., \& Jex, S. M. (1998). Development of four self-report measures of job stressors and strain: Interpersonal conflict at work scale, organizational constraints scale, quantitative workload inventory, and physical symptoms inventory. Journal of Occupational Health Psychology, 3, 356-367. doi: 10.1037/1076-8998.3.4.356

Spector, P. E., Coulter, M. L., Stockwell, H.G., \& Matz, M.W. (2007). Perceived violence climate: A new construct and its relationship to workplace physical violence and verbal aggression, and their potential consequences. Work \& Stress, 21, 117-130.

Stattin, H. \& Kerr, M. (2000). Parental monitoring: A reinterpretation. Child Development, 71, 1072-1085.

Stewart, W. \& Barling, J. (1996). Fathers' work experiences effect children's behaviors via job-related affect and parenting behaviors. Journal of Organizational Behavior, 17, 221-232.

Stoetzer, U., Ahlberg, G., Johansson, G., Bergman, P., Hallsten, L., Forsell, Y., \& Lundberg, I. (2009). Problematic interpersonal relationships at work and depression: A Swedish prospective cohort study. Journal of Occupational Health, $51,144-151$.

Story, L. B. \& Repetti, R. (2006). Daily occupational stressors and marital behavior. Journal of Family Psychology, 20, 690-700. doi: 10.1037/0893-3200.20.4.690

Taylor, J. L., \& Rew, L. (2011). A systematic review of the literature: workplace violence in the emergency department. Journal of clinical nursing, 20(7-8), 1072-1085. 
ten Brummelhuis, L. L. \& Bakker, A. B. (2012). A resource perspective on the workhome interface: The work-home resources model. American Psychologist, 67, 545-556. doi: 10.1037/a0027974

Tepper, B. J. (2000). Consequences of abusive supervision. Academy of Management Journal, 43, 178-190. doi: 10.2307/1556375

Thoits, P. A. (1994). Stressors and problem-solving: The individual and psychological activist. Journal of Health and Social Behavior, 35, 143-160. doi:10.2307/2137362

Trépanier, S.-G., Fernet, C., \& Austin, S. (2015). A longitudinal investigation of workplace bullying, basic need satisfaction, and employee functioning. Journal of Occupational Health Psychology, 20, 105-116.

van de Heijden, B. I. J. M., Demerouti, E., Bakker, A. B., \& The NEXT study group coordinated by H.-M. Hasselhorn. (2008). Work-home interference among nurses: Reciprocal relationships with job demands and health. Journal of Advanced Nursing, 62, 572 - 584. doi: 10.1111/j.1365-2648.2008.04630.x

van der Linden, D., Keijsers, G. P. J., Eling, P., \& van Schaijk, R. (2005). Work \& Stress, 19, 23-36. doi: 10.1080/02678370500065275

Vinokur, A. D. \& Schul, Y. (2002). The web of coping resources and pathways to reemployment following a job loss. Journal of Occupational Health Psychology, 7, 68-83.

Volmer, J., Binnewies, C., Sonnentag, S., \& Niessen, C. (2012). Do social conflicts with customers at work encroach upon our private lives? A diary study. Journal of Occupational Health Psychology, 17, 304-315. doi: 10.1037/a0028454

Wadsworth, E., Moss, S., Simpson, S., \& Smith, A. (2003). Preliminary investigation of the association between psychotropic medication use and accidents, minor injuries and cognitive failures. Human Psychopharmacology: Clinical and Experimental, $18,535-540$.

Wallace, J. C., \& Chen, G. (2005). Development and validation of a work-specific measure of cognitive failure: Implications for occupational safety. Journal of Occupational and Organizational Psychology, 78, 615-632.

Wallace, J. C., Edwards, B. D., Arnold, T., Frazier, M. L., \& Finch, D. M. (2009). Work stressors, role-based performance, and the moderating influence of organizational performance. Journal of Applied Psychology, 94, 254-262. doi: $10.1037 / \mathrm{a} 0013090$

Wallace, J. C. \& Vodanovich, S. J. (2003a). Can accidents and industrial mishaps be predicted? Investigating workplace performance. Journal of Business and Psychology, 17, 503-514. 
Wallace, J. C. \& Vodanovich, S. J. (2003b). Workplace safety performance: Conscientiousness, cognitive failure, and their interaction. Journal of Occupational Health Psychology, 8, 316-327. doi: 10.1037/1076-8998.8.4.316

Wang, S., Repetti, R. L., \& Campos, B. (2011). Job stress and family social behavior: The moderating role of neuroticism. Journal of Occupational Health Psychology, $16,441-456$.

Xanthopoulou, D., Bakker, A. B., Demerouti, E., \& Schaufeli, W. B. (2007). The role of personal resources in the job-demands resources model. International Journal of Stress Management, 14, 121-141.

Yang, L. Q. (2009). Aggression and its consequences in Nursing: A more complete story by adding its social context. Unpublished doctoral dissertation at the University of South Florida, Tampa, FL.

Yang, L. Q., Caughlin, D. E., Gazica, M. W., Truxillo, D. M., \& Spector, P. E. (2014). Workplace mistreatment climate and potential employee and organizational outcomes: A meta-analytic review from the target's perspective. Journal of Occupational Health Psychology, 19, 315 - 335.

Yragui, N. L., Hammer, L., \& Demsky, C. A. (June 2014). Linking workplace violence to employee health: The moderating role of family supportive supervisory behaviors (FSSB). Paper presented as a part of the symposium entitled "Work-life challenges and social support: Social workers and direct care workers" at the Work and Family Researchers' Network Annual Conference, New York City.

Yragui, N. L., Silverstein, B. A., Foley, M., Johnson, W., \& Demsky, C. (2012). The Washington State Psychiatric Hospital Work, Stress, and Health Project: Final Report to Washington DSHS Mental Health Division and Western State Hospital. Unpublished Technical Report.

Zapf, D., Dormann, C., \& Frese, M. (1996). Longitudinal studies in organizational stress research: A review of the literature with reference to methodological issues. Journal of Occupational Health Psychology, 1, 145-169.

Zeller, A., Dassen, T., Kok, G., Needham, I. \& Halfens, R. J. (2012). Factors associated with resident aggression toward caregivers in nursing homes. Journal of Nursing Scholarship, 44(3), 249-257.

Zeller, A., Hahn, S., Needham, I., Kok, G., Dassen, T., \& Halfens, R. J. (2009). Aggressive behavior of nursing home residents toward caregivers: a systematic literature review. Geriatric Nursing, 30(3), 174-187.

Zijlstra, F. R. H. \& Sonnentag, S. (2006). After work is done: Psychological perspectives on recovery from work. European Journal of Work and Organizational Psychology, 15, 129-138. doi: 10.1080/13594320500513855 
Zoccola, P. M., Figueroa, W. S., Rabideau, E. M., Woody, A., \& Benencia, F. (2014). Health Psychology, 33, 1606-1609. doi: 10.1037/hea0000019

Zohar, D. (1980). Safety climate in industrial organizations: Theoretical and applied implications. Journal of Applied Psychology, 65, 96-102.

Zohar, D. (2003). Safety climate: Conceptual and measurement issues. In J. C. Quick \& L. E. Tetrick (Eds.), Handbook of occupational health psychology (pp. 123-142). Washington, DC: American Psychological Association.

Zohar, D. \& Luria, G. (2003). The use of supervisory practices as leverage to improve safety behavior: A cross-level intervention model. Journal of Safety Research, 34, 567-577. doi: 10.1016/j.jsr.2003.05.006

Zohar, D. \& Luria, G. (2005). A multilevel model of safety climate: Cross-level relationships between organization and group-level climates. Journal of Applied Psychology, 90, 616-628. doi: 10.1037/0021-9010.90.4.616

Zohar, D. \& Polachek, T. (2014). Discourse-based intervention for modifying supervisory communication as leverage for safety climate and performance improvement: A randomized field study. Journal of Applied Psychology, 99, 113 124. doi: $10.1037 / \mathrm{a} 0034096$ 
Appendix: Items for Study \#3

Workplace Incivility Scale (WIS; Cortina, Magley, Williams, \& Langhout, 2001)

Directions: During the past six months while employed by the USDA Forest Service, have you been in a situation where any of your supervisors:

1. Put you down or were condescending to you?

2. Paid little attention to your statement or showed little interest in your opinion?

3. Made demeaning or derogatory remarks about you?

4. Addressed you in unprofessional terms, either publicly or privately?

5. Ignored or excluded you from professional camaraderie?

6. Doubted your judgment on a matter over which you have responsibility?

7. Made unwanted attempts to draw you into a discussion of personal matters?

Response Options: 1 (never) to 5 (most of the time)

Directions: During the past six months while employed by the USDA Forest Service, have you been in a situation where any of your coworkers:

1. Put you down or were condescending to you?

2. Paid little attention to your statement or showed little interest in your opinion?

3. Made demeaning or derogatory remarks about you?

4. Addressed you in unprofessional terms, either publicly or privately?

5. Ignored or excluded you from professional camaraderie?

6. Doubted your judgment on a matter over which you have responsibility?

7. Made unwanted attempts to draw you into a discussion of personal matters?

Response Options: 1 (never) to 5 (most of the time)

Cognitive Failure (Wallace \& Chen, 2005)

Directions: Please indicate how frequently you experienced each of these in the past $\underline{\text { six }}$ months using the response scale provided.

Response Options: 1 (never), 2 (once in awhile), 3 (about as often as not), 4 (often), 5 (always)

1. How often did you fail to notice postings or notices on the facilities bulletin board(s) or email system at work?* 
2. How often did you not fully listen to instructions at work?*7

3. How often did you day-dream when you ought to be listening to somebody at work?

4. How often did you not focus your attention on work activities at work?

5. How often were you easily distracted by coworkers at work?

Work-Related Rumination (Cropley, Michalianou, Pravettoni, \& Millward, 2012)

Directions: Please indicate how frequently you experienced each of these in the past six months.

1. Did you become tense when you think about work-related issues during your free time?

2. Were you annoyed by thinking about work-related issues when not at work?

3. Were you irritated by work issues when not at work?

4. Were you become fatigued by thinking about work-related issues during your free time?

5. Were you troubled by work-related issues when not at work?

Response Options: 1 = very seldom or never; $2=$ seldom; $3=$ sometimes; $4=$ often; $5=$ very often or always

\section{Mindfulness - Five Facet Mindfulness Questionnaire - (Baer, Smith, Hopkins, Krietemeyer, \& Toney, 2006)}

Directions: Please rate each of the following statements using the response options provided. Select the option that best describes what is generally true for you.

1. I perceive my feelings and emotions without having to react to them.

2. I watch my feelings without getting lost in them.

3. In difficult situations, I can pause without immediately reacting.

4. Usually when I have distressing thoughts or images, I am able just to notice them without reacting.

5. Usually when I have distressing thoughts or images, I feel calm soon after.

6. Usually when I have distressing thoughts or images, I "step back" and am aware of the thought or image without getting taken over by it.

7. Usually when I have distressing thoughts or images, I just notice them or let them go.

Response Options: 1 = never or very rarely; $2=$ rarely true $; 3=$ sometime $; 4=$ often; 5 $=$ very often or always true

\footnotetext{
${ }^{7}$ Note: Cognitive failure items marked with an asterisk were removed from the final scale used in Study 3 in order to improve the reliability of the overall scale.
} 
Safety Climate - (Neal, Griffin, \& Hart, 2000)

Directions: Please indicate your agreement or disagreement with each statement by selecting the appropriate response option.

1. Management places a strong emphasis on workplace health and safety.

2. Safety is given a high priority by management.

3. Management considers safety to be important.

Response Options: 1(Strongly disagree) to 5 (Strongly agree)

Safety Behaviors - (Neal, Griffin, \& Hart, 2000)

Directions: Generally, to what degree to you agree with the following statements?

\section{Safety Compliance}

1. I use all the necessary safety equipment to do my job.

2. I use the correct safety procedures for carrying out my job.

3. I ensure the highest levels of safety when I carry out my job.

\section{Safety Participation}

1. I promote the safety program within the organization.

2. I put in extra effort to improve the safety of the workplace.

3. I voluntarily carry out tasks or activities that help to improve workplace safety.

Response Options: 1(Strongly disagree) to 5 (Strongly agree)

Accidents (Hayes, Perander, Smecko, \& Trask, 1998)

1. How many accidents have you experienced in the last 12 months that were:

a. reported accidents (reported to the supervisor)?

b. unreported accidents (not reported to the supervisor)?

c. near accidents (something that could have caused an injury but did not)?

Injuries - (Barling, Kelloway, \& Iverson, 2003)

2. Have you experienced an injury on the job in the last year?

3. If yes, how many days were you off of work as a result of your injury?

\section{DEMOGRAPHIC VARIABLES}

1. What is your age?

2. What gender do you identify with (Male, Female, Other) 
3. What is your ethnicity? (African American, White (non-Hispanic), Hispanic/Latino, Native American, Asian, Native Alaskan or Pacific Islander, Other)

4. What is the highest level of education you have completed? (High school diploma/GED, some college, 2-year college degree (Associate's), 4-year college degree (Bachelor's), Advanced degree-Master's or other)

5. Would you classify your job as: (supervisory - staff report to you; nonsupervisory)

6. How many years have you worked for the USDA Forest Service?

7. How many years have you worked in your current position?

8. On average, how many days per week do you normally work? (1-7)

9. On average, how many hours per week do you normally work?

10. On average, how many hours of overtime per week do you normally work?

11. What is your current relationship status? (Single-never been married, Dating someone, Married, Living with a partner, Divorced, Widowed)

12. How many children under 18 do you have that are living with you at least half time?

Note: Answers to the following questions are used as a grouping function for the purpose of data analysis only and will not be used to identify you as an employee.

1. What area of the USDA Forest Service do you primarily work for? (wildland firefighting, parks and recreation, ETC.)

2. What National Forest do you work in? (Apache-Sitgreaves; Coconino; Coronado; Kaibab; Prescott; Tonto; Carson; Ciboloa; Gila; Lincoln; Sante Fe; N/A)

3. What Ranger District do you work in? (Alpine; Clifton; Black Mesa; Springerville; Lakeside; Flagstaff; Red Rock; Mogollon Rim; Douglas; Nogales; Sierra Vista; Safford; Santa Catalina; North Kaibab; Tusayan; Williams; Bradshaw; Chino Valley; Verde; Prescott Fire Center; Globe; Mesa; Payson; Pleasant Valley; Tonto Basin; Canjilon; El Rito; Jicarilla; Camino Real; Tres Piedras; Questa; Black Kettle; Kiowa and Rita Blanca; Magdalena; Mountainair; Mt. Taylor; Sandia; Black Range; Glenwood; Quemado; Gila Cliff Dwellings National Monument and Gila Visitors Center; Reserve; Silver City; Wilderness; Smokey Bear; Sacramento; Guadalupe; Coyote; Cuba; Jemez; Pecos; Las Vegas; Espanola; NA) 